\title{
EFFECTS OF SUPPORT STRUCTURE GEOMETRY ON SLM INDUCED RESIDUAL STRESSES IN OVERHANGING FEATURES
}

\author{
A Thesis \\ presented to \\ the Faculty of California Polytechnic State University, \\ San Luis Obispo
}

\author{
In Partial Fulfillment \\ of the Requirements for the Degree \\ Master of Science in Mechanical Engineering
}

by

Ryan Baskett

September 2017 
(C) 2017

Ryan Baskett

ALL RIGHTS RESERVED 


\section{COMMITTEE MEMBERSHIP}

TITLE:

Effects of Support Structure Geometry on SLM Induced Residual Stresses in Overhanging Features

AUTHOR: $\quad$ Ryan Baskett

DATE SUBMITTED: September 2017

COMMITTEE CHAIR: Kim Shollenberger, Ph.D.

Professor of Mechanical Engineering

COMMiTTEe MEMBER: Christopher Pascual, Ph.D, P.E.

Professor of Mechanical Engineering

COMMITTEE MEMBER: Joshua Webb, Ph.D.

Defense Technology Engineering Division Lawrence Livermore National Laboratory 


\begin{abstract}
Effects of Support Structure Geometry on SLM Induced Residual Stresses in Overhanging Features

Ryan Baskett
\end{abstract}

Selective laser melting (SLM) is a new and rapidly developing manufacturing method for producing full-density, geometrically complex metal parts. The SLM process is time and cost effective for small-scale production; however, wide-spread adoption of this technique is severely limited by residual stresses that can cause large deformations and in-process build failures. The issues associated with residual stress accumulation are most apparent in parts with overhanging features. Due to the complexity of the SLM process, the accumulation of residual stresses is difficult to assess a priori. The deformations and in-process failures caused by residual stress accumulation often lead to an expensive and time consuming iterative manufacturing process.

To aid in the development of general SLM design guidelines for overhanging features, the effect of varying two support structure design parameters on residual stress accumulation were investigated. A part-scale thermo-mechanical finite element model was implemented using Diablo, a multi-physics finite element code developed by Lawrence Livermore National Laboratory (LLNL), and trends observed in the model were validated experimentally.

By comparing the distribution and magnitude of residual stresses, it was determined that reducing cooling rate gradients in overhanging features reduces the resulting residual stresses. Additionally, it was shown that volume effective material properties can be used to reduce computational costs in computational models of the SLM process. 


\section{ACKNOWLEDGMENTS}

I would first like to thank my thesis advisor, Dr. Shollenberger, for her continued support on this project. From the initial problem definition through to interpreting the final results, Dr. Shollenberger provided invaluable insight and kept me on track.

I would also like to thank my other committee members, Dr. Pascual and Dr. Webb. It was in Dr. Pascual's Thermodynamics 1 class that I first found my passion for thermal sciences. Since then Dr. Pascual has helped my through my academic career, from advising me on class selection to helping me revise my thesis.

Dr. Webb's expertise with Diablo was instrumental to this work. Without Dr. Webb's support I would not have been able to access the incredible computational resources of Lawrence Livermore National Laboratory or gain the skills necessary to use Diablo.

I would also like to acknowledged everyone at LLNL and Cal Poly who supported me in this project, especially Dr. Rishi Ganeriwala, Stephen Knaus, and Dr. Xuan Wang. Dr. Ganeriwala's assistance with Diablo, and other LLNL software, was invaluable to both this research and past research projects.

Stephen Knaus had a hand in helping me select my thesis project, and he also coordinated aspects of the experimental validation conducted at LLNL facilities.

Dr. Wang provided me with all the resources I needed for the experimental validation, and he also advised me on the design of the experiment.

Finally, I would express my profound gratitude to my family. They have supported and encouraged me throughout my entire life and this accomplishment would not have been possible without them.

- Ryan Baskett 


\section{TABLE OF CONTENTS}

LIST OF TABLES . . . . . . . . . . . . . . . . . . . . . . . . . . . . . $\ldots$. viii
LIST OF FIGURES
CHAPTER

1 Introduction . . . . . . . . . . . . . . . . . . 1

1.1 The SLM Process . . . . . . . . . . . . . . . . . . 1

1.2 The Origin of Residual Stress . . . . . . . . . . . . . . 3

2 Previous Work . . . . . . . . . . . . . . . . . . . 9

2.1 Overview ............................. 9

2.2 Support Structure Design Considerations . . . . . . . . . . . . . 9

2.2.1 Contact Area . . . . . . . . . . . . . . . . . . . . 11

2.2.2 Support Base . . . . . . . . . . . . . . . . . . 15

2.3 Influence of Process Parameters . . . . . . . . . . . . . . . . 22

2.3.1 Scan Speed . . . . . . . . . . . . . . . 22

2.3.2 Scan Strategy . . . . . . . . . . . . . . . . 23

2.3 .3 Laser Power . . . . . . . . . . . . . . . . . . . . . . . . 24

2.3.4 Layer Thickness . . . . . . . . . . . . . . . . . 25

2.3.5 Preheating . . . . . . . . . . . . . . 25

2.3.6 Remelting ................... . . 26

2.3.7 Post-Process Heat Treating . . . . . . . . . . . . . 26

2.3.8 Process Parameter Summary . . . . . . . . . . . . . . 26

2.4 Process Modeling . . . . . . . . . . . . . . . . 26

2.4.1 Thermal Models . . . . . . . . . . . . . . . 27

2.4.2 Thermo-Mechanical Models . . . . . . . . . . . . 27

2.4 .3 Material Models . . . . . . . . . . . . . . . 29

3 Model Development . . . . . . . . . . . . . . . . . . 30

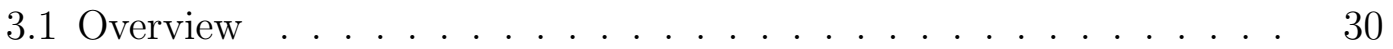


3.2 Thermal Model . . . . . . . . . . . . . . . . . . . . . . . . . 32

3.3 Thermo-Mechanical Model . . . . . . . . . . . . . . . . 35

3.4 Material Models . . . . . . . . . . . . . . . . . 36

3.4 .1 Material Model 1 . . . . . . . . . . . . . . . . 36

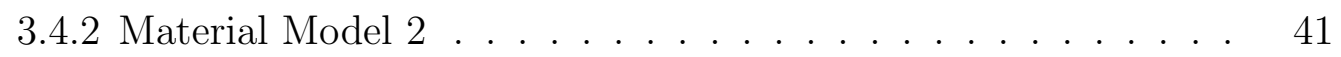

3.4 .3 Material Model 3 . . . . . . . . . . . . . . . . . . 45

3.4 .4 Material Model $4 \ldots \ldots$. . . . . . . . . . . . . . . . . . . 48

3.5 Numerical Implementation . . . . . . . . . . . . . . . . . 49

3.5.1 Thermal Numerical Implementation . . . . . . . . . . . . 49

3.5.2 Thermal Initial/Boundary Conditions . . . . . . . . . . . 51

3.5.2.1 Initial condition . . . . . . . . . . . . . . . 51

3.5.2.2 Boundary conditions . . . . . . . . . . . . . 52

3.5.3 Thermo-Mechanical Numerical Implementation . . . . . . . 53

3.5.4 Solid Mechanics Initial/Boundary Conditions _. . . . . . . 54

3.5.4.1 Initial condition . . . . . . . . . . . . . . . 54

3.5.4.2 Boundary conditions . . . . . . . . . . . . . 54

3.6 Domain Discretization . . . . . . . . . . . . . 54

3.7 Mesh Dependence . . . . . . . . . . . . . . . . . . 57

3.8 Experimental Validation $\ldots \ldots \ldots \ldots$

4 Results \& Discussion . . . . . . . . . . . . . . . . 66

4.1 In-Plane Residual Stresses . . . . . . . . . . . . . . . 67

4.1 .1 Results . . . . . . . . . . . . . . . 6 67

4.1 .2 Discussion . . . . . . . . . . . . . . . . . . . 69

4.2 Axial Residual Stresses . . . . . . . . . . . . . . . . 71

4.2 .1 Results . . . . . . . . . . . . . . . . . 71

4.2 .2 Discussion . . . . . . . . . . . . . . . . . . . . . 72

4.3 Effect of Contact Area Parameters _ . . . . . . . . . . . 78

4.3 .1 Results . . . . . . . . . . . . . . . . . . 78

4.3 .2 Discussion . . . . . . . . . . . . . . . . . . . . . 83

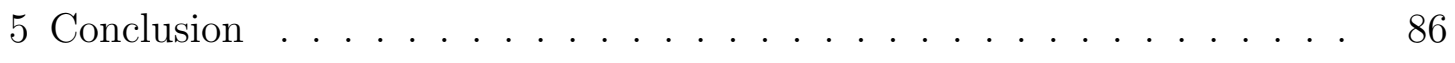

5.1 Summary . . . . . . . . . . . . . . 86

5.2 Future Work . . . . . . . . . . . . . . . . 87

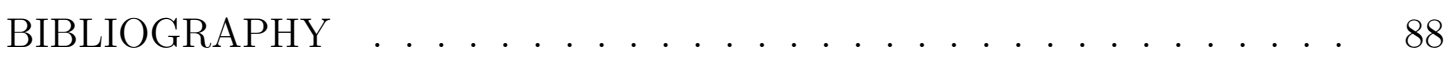
APPENDICES

A Additional Experimental Validation Results . . . . . . . . . . . 97 


\section{LIST OF TABLES}

Table $\quad$ Page

3.1 Factorial Study Design Points . . . . . . . . . . . . . . . . . 31

4.1 Factorial Study Maximum Residual Stress Results . . . . . . . . . . . 79 


\section{LIST OF FIGURES}

Figure

Page

1.1 SLM Build Process Mechanics [40] . . . . . . . . . . . . . 2

1.2 Thermally Induced Residual Stresses via Quenching of a Metal Ingot $[52] \ldots \ldots \ldots \ldots \ldots \ldots \ldots$. . . . . . . . . . . . . . . 4

1.3 TGM Inducing Residual Stresses [40] . . . . . . . . . . . . . . . 5

1.4 a) Temperature Distribution in SLM in the Build Direction b) Resulting Residual Stress Distribution at Three Stages [33] . . . . . . 5

1.5 a) Residual Stress Distribution After Adding Two Layers of Melted Powder b) Residual Stress Distribution After Final Layer [28] . . . . 7

2.1 Literature Review Organizational Chart . . . . . . . . . . . . . . 9

2.2 Support Structure Regions . . . . . . . . . . . . . . . . . . . 10

2.3 Contact Area Parameters . . . . . . . . . . . . . . . . . 11

2.4 a) Dimensions of Part b) Block Support Parameters c) Tooth Parameters $[42] \ldots \ldots \ldots \ldots \ldots$

2.5 Support Structure Warping Comparison [33] . . . . . . . . . . . 13

2.6 Transient Temperature Distribution in Overhang Layer for Asymmetric Contact Points (a-c) and Symmetric Contact Points (d-f) at 3 Time Intervals [13] . . . . . . . . . . . . . . . . . . . . . 14

2.7 a) Model Geometry b) Model Boundary Conditions [7] . . . . . . . . 17

2.8 Geometric Variations Considered [7] . . . . . . . . . . . . . 17

2.9 a) Deformation After Cooling ( $\mathrm{L}=15[\mathrm{~mm}])$ b)Comparison of Deformations Along Top Surface for Varying Overhang Length [7] . . . . . 18

2.10 Comparison of Deformations Along Top Surface for Varying Support Width $[7] \ldots \ldots \ldots \ldots$

2.11 a) Deformation After Cooling (Gap $=0.63[\mathrm{~mm}])$ b)Comparison of Deformations Along Top Surface [7] . . . . . . . . . . . . . . . 19

2.12 Powder Bed Temperature Distribution Comparison [7] . . . . . . . . 20

2.13 Comparison of Deformations Along Top Surface for Varying Gap Width

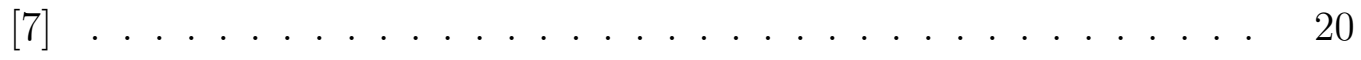


Figure

2.14 Binary Phase Diagram Containing Material A and B [51] . . . . . . . 21

2.15 Scan Speed Effect on Warping [54] . . . . . . . . . . . . . 23

2.16 Critical Incline Angle and Energy Input Correlation [54] . . . . . . . 25

2.17 Global Model Scan Strategy [31] . . . . . . . . . . . . . . . . 28

3.1 Side View of Test $4 \ldots \ldots$. . . . . . . . . . . . . 31

3.2 Laser Radiation Transfer in a Powder Layer on a Substrate [15] . . . 34

3.3 Uniform Laser Heat Source Model . . . . . . . . . . . . . . . . . . . . 34

3.4 Side View of Test $4 \ldots \ldots$. . . . . . . . . . . . 36

3.5 Material 1 Temperature Dependent Thermal Conductivity [14] . . . . 38

3.6 Material 1 Temperature Dependent Specific Heat [14] . . . . . . . . . 38

3.7 Material 1 Temperature Dependent Young's Modulus [14] . . . . . . . 39

3.8 Material 1 Temperature Dependent Yield Stress [14] . . . . . . . . . 39

3.9 Material 1 Temperature Dependent Isotropic Hardening Modulus [14] 40

3.10 Material 1 Temperature Dependent Coefficient of Thermal Expansion

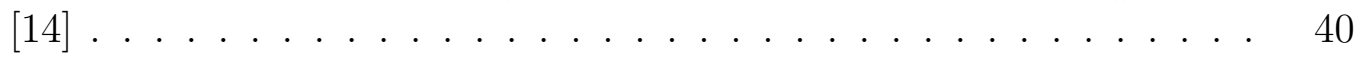

3.11 Material 2 Temperature Dependent Thermal Conductivity [14] . . . . 42

3.12 Material 2 Temperature Dependent Specific Heat [14] . . . . . . . . . 42

3.13 Material 2 Temperature Dependent Young's Modulus [14] . . . . . . 43

3.14 Material 2 Temperature Dependent Yield Stress [14] . . . . . . . . . 43

3.15 Material 2 Temperature Dependent Isotropic Hardening Modulus [14] 44

3.16 Material 2 Temperature Dependent Coefficient of Thermal Expansion

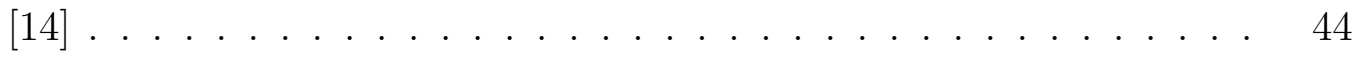

3.17 Block Support Structure Meshed Cross Section . . . . . . . . . . 45

3.18 Volume Effective Support Structure Meshed Cross Section . . . . . . 46

3.19 Volume Effective Validation Loading Case . . . . . . . . . . . . . . . 47

3.20 Volume Effective Validation Average Bottom Surface Temperature . . 47

3.21 Volume Effective Validation Average Bottom Surface Displacement . 48

3.22 Global Model Laser Path . . . . . . . . . . . . . . . . . . . . 51

3.23 Initial Temperature Distribution . . . . . . . . . . . . . 52

3.24 Thermo-Mechanical Coupling Algorithm [16] . . . . . . . . . 53

3.25 Isometric View of Test 4 Mesh . . . . . . . . . . . . . . . 55 
Figure

3.26 Side View of Test 4 Mesh . . . . . . . . . . . . . . . . 56

3.27 Front View of Test 4 Mesh . . . . . . . . . . . . . . . . 56

3.28 Thermal Mesh Dependence in the Part . . . . . . . . . . . . . . 57

3.29 Thermal Mesh Dependence in the Support . . . . . . . . . . . . . 58

3.30 Quantitative Measure of Thermal Mesh Dependence . . . . . . . . . . 59

$3.31 \sigma_{x x}$ Mesh Dependence Under Tensile Load . . . . . . . . . . . . . . 60

$3.32 \sigma_{y y}$ Mesh Dependence Under Tensile Load . . . . . . . . . . . . . . . 60

$3.33 \sigma_{z z}$ Mesh Dependence Under Tensile Load . . . . . . . . . . . . . . 61

3.34 Residual Stress Mesh Dependence . . . . . . . . . . . . . . . . . . 62

3.35 Experimental Build Plate Layout . . . . . . . . . . . . 63

3.36 Detached Support . . . . . . . . . . . . . . . 63

3.37 Test 1 Experimental Validation at $\mathrm{Y} \approx 19[\mathrm{~mm}] \ldots . . . . . .664$

3.38 Test 2 Experimental Validation at $\mathrm{Y} \approx 19[\mathrm{~mm}] \ldots . . . . . .65$

4.1 Overhang Section Dimensions . . . . . . . . . . . . 66

4.2 Test $2 \sigma_{x x} \ldots \ldots \ldots \ldots \ldots$

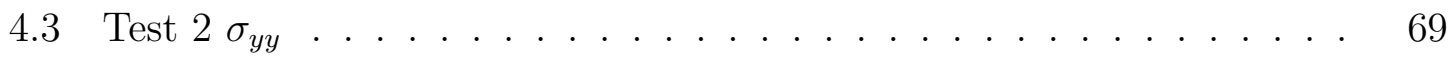

4.4 In-Plane Residual Stress Distribution . . . . . . . . . . . . . . 70

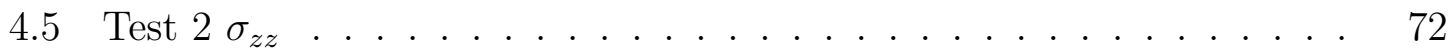

4.6 Part Temperature Contour 1 . . . . . . . . . . . . . . 73

4.7 Part Temperature Contour 2 . . . . . . . . . . . . . . . 74

4.8 Temperature in Elements A and B . . . . . . . . . . . . 74

4.9 1D Illustration of the Molten Layer Cool-Down Mechanism . . . . . 76

4.10 Axial Residual Stress in Elements A and B . . . . . . . . . . . . . 77

4.11 Part Temperature Contour 3 . . . . . . . . . . . . . . . 78

4.12 Top Length and Tooth Spacing Effect on Tensile $\sigma_{x x}$. . . . . . . . . 79

4.13 Top Length and Tooth Spacing Effect on Compressive $\sigma_{x x}$. . . . . 80

4.14 Top Length and Tooth Spacing Effect on Tensile $\sigma_{y y} \quad \ldots . . .81$

4.15 Top Length and Tooth Spacing Effect on Compressive $\sigma_{y y}$. . . . . . 81

4.16 Top Length and Tooth Length Effect on Tensile $\sigma_{z z} \ldots$. . . . . . . 82

4.17 Top Length and Tooth Length Effect on Compressive $\sigma_{z z} \ldots$. . . . 83

4.18 Maximum Tensile and Minimum Compressive Residual Stresses . . . 84 
Figure

4.19 Normalized Maximum Tensile and Minimum Compressive Residual Stresses . . . . . . . . . . . . . . . . . . 84

A.1 Test 1 Experimental Validation at $\mathrm{Y} \approx 11[\mathrm{~mm}] \ldots \ldots \ldots . \ldots 97$

A.2 Test 2 Experimental Validation at $\mathrm{Y} \approx 11[\mathrm{~mm}] \ldots \ldots \ldots \ldots$

A.3 Test 1 Experimental Validation at $\mathrm{Y} \approx 12[\mathrm{~mm}] \ldots \ldots \ldots . \ldots 98$

A.4 Test 2 Experimental Validation at $\mathrm{Y} \approx 12[\mathrm{~mm}] \ldots \ldots \ldots$

A.5 Test 1 Experimental Validation at $\mathrm{Y} \approx 14[\mathrm{~mm}] \ldots \ldots . \ldots . . .99$

A.6 Test 2 Experimental Validation at $\mathrm{Y} \approx 14[\mathrm{~mm}] \ldots \ldots \ldots$

A.7 Test 1 Experimental Validation at $\mathrm{Y} \approx 15[\mathrm{~mm}] \ldots \ldots \ldots \ldots$

A.8 Test 2 Experimental Validation at $\mathrm{Y} \approx 15[\mathrm{~mm}] \ldots \ldots \ldots \ldots \ldots$

A.9 Test 1 Experimental Validation at $\mathrm{Y} \approx 18[\mathrm{~mm}] \ldots \ldots \ldots \ldots \ldots$

A.10 Test 2 Experimental Validation at $\mathrm{Y} \approx 18[\mathrm{~mm}] \ldots \ldots \ldots \ldots$ 


\title{
CHAPTER 1
}

\author{
Introduction
}

\subsection{The SLM Process}

Selective Laser Melting (SLM) is an emerging additive manufacturing (AM) technique that enables the rapid production of geometrically complex full-density metal parts [4]. Like all AM processes, SLM created objects from 3D model data by joining material in a layer-by-layer manner [1]. According to the American Society for Testing and Materials (ASTM), the SLM process is a powder bed fusion process because it uses a fine metal powder as raw material [1].

Due to a high volume of concurrent research and development, the SLM process is referred to by several common names including LaserCUSING ${ }^{\circledR}$, Direct Metal Laser Sintering (DMLS), Laser Sintering (LS), and Laser Beam Melting (LMB) [42, 61]. While melting and sintering refer to two different binding mechanisms between the powder particles, all of these processes describe the same basic procedure which is illustrated in Figure 1.1.

The wiper, a roller which spreads the powder onto the build plate, deposits a 20 $100[\mu \mathrm{m}]$ layer of powder $[12,40]$ over the base plate in a protective gas environment and the layer is selectively fused by a laser. The base plate then moves down one layer thickness, the wiper deposits a new layer, and the process is repeated until the part is finished [37]. 


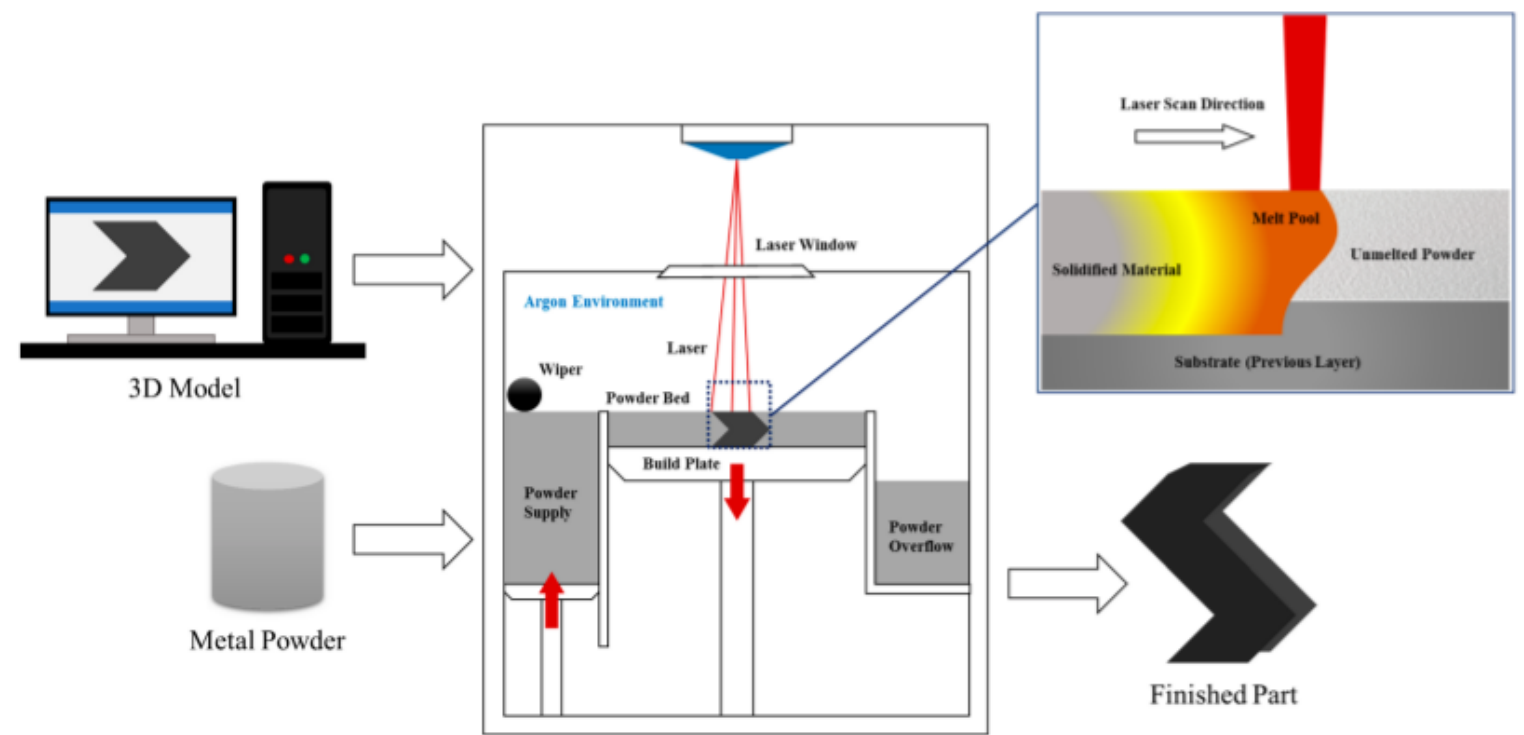

Figure 1.1 - SLM Build Process Mechanics [40]

As noted above, melting is the fusing of fully molten particles, while sintering is the fusing of only partially molten particles [38]. According to ASTM F2792-12a [1] sintering is a misnomer when it is is used to describe powder bed fusion AM processes because powder bed fusion typically involves full or partial melting. Not all powder bed fusion processes involve full or partial melting so sintering cannot always be assumed to mean melting [38, 44].

For the purpose of this study, the process described above will be referred to as SLM and will entail a "metallic powder free of binding and flux agents" being "heated to its melting temperature throughout the layer thickness" in a "protective gas environment" as described in the U.S. patent for the SLM process [37].

SLM is a time and cost effective small-scale production or prototyping method for full-density, geometrically complex metal parts. The cost and time savings makes the SLM process appealing to the aerospace, medical, and automotive industries. Unfortunately, the wide-spread adoption of this technology is severely limited by residual 
stresses introduced during the SLM process [24, 25, 38, 40, 58, 62]. These residual stresses, which are produced by the expansion and contraction of the previously solidified layers can lead to localized deformation and in-process failure [24, 25, 26, 40, 58].

To prevent in-process failure in overhanging features where deformation is most severe $[6,38,40]$, support structures are used to anchor the overhang to the build plate and to conduct heat away from the part $[24,62]$. Currently, the design of support structures is based on various rules-of-thumb gained from application specific experiences and not based on general support structure design theory [40,62]. When a support structure designed using these rules-of-thumb fails to prevent fractures in a part, a practitioner is forced to use a trial and error manufacturing process which wastes both time and material $[57,62]$.

\subsection{The Origin of Residual Stress}

According to Withers [55], residual stresses are stresses that remain inside a body that is stationary and at equilibrium with its surroundings. Residual stresses are classified in three types based on the characteristic length over which they reach equilibrium. Type I residual stresses act over the characteristic length of the whole body, and are referred to as macroscopic residual stresses. Type II and Type III are referred to as microscopic residual stresses and equilibrate over much smaller length scales. Type II stresses originate from local phenomena, such as different material phases, while Type III result from defects in the crystalline structure, and therefore have a smaller characteristic length than Type II residual stresses $[38,52,56]$.

Residual stresses are the result of non-homogeneous plastic strains in a material caused by thermal or mechanical loads [52]. Thermal residual stresses are introduced

by larger thermal gradients, which are inherently present in the SLM process [38]. Figure 1.2 illustrates the introduction of residual stresses in a hot metal ingot being 
quenched. Initially the ingot is at a uniform temperature (left most image). When the ingot is introduced to a much colder environment, the outside of the ingot cools and contracts faster than the interior of the ingot. The contracting region of the ingot compresses the interior of the ingot (2nd image from the left). Due to its elevated temperature, the interior of the ingot has a lower yield point and plastically yields (3rd image from the left). After the ingot fully cools, the plastically compressed interior region contracts and exerts a compressive force on the exterior region (right most image) [52].
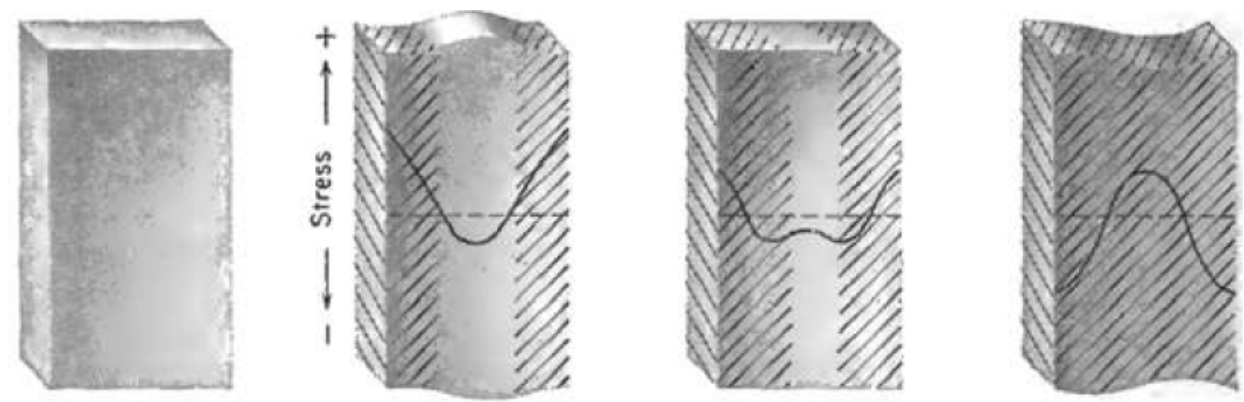

Figure 1.2 - Thermally Induced Residual Stresses via Quenching of a Metal Ingot [52]

In SLM, there are two mechanisms that cause residual stresses. The first mechanism is known as the temperature gradient mechanism (TGM) and results from the large thermal gradients around the laser spot [38]. The TGM, shown in Figure 1.3, is analogous to the process shown in Figure 1.2. During heating, the expansion of the hot upper layer is restricted by the colder layer below, introducing compressive plastic strain in the upper layer. Upon cooling, the upper layer contracts, resulting in a tensile stress in the upper layer and a compressive stress in the lower layer. 

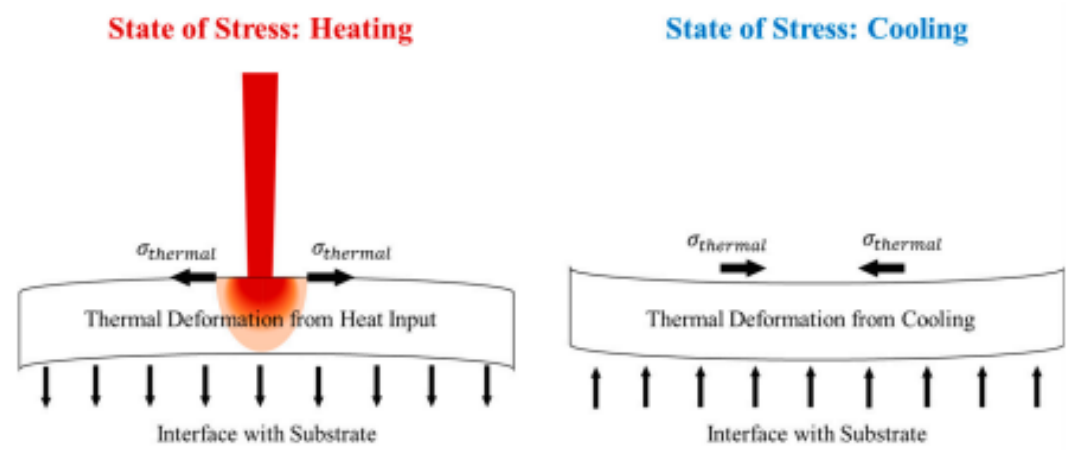

Figure 1.3 - TGM Inducing Residual Stresses [40]

The second mechanism is the cool-down phase of the molten top layers. When the molten layer solidifies and cools, it contracts; however, this contraction is restricted by the surrounding colder material which leads to tensile stress in the newly solidified region and compressive stress in the surrounding region. Figure 1.4 represents the temperature distribution in the SLM process as a function of depth from the top surface and the resulting residual stresses at three stages. $T_{m}, T_{p}$, and $T_{n}$ are the melting point, the plastic point, and the ambient temperature, respectively.
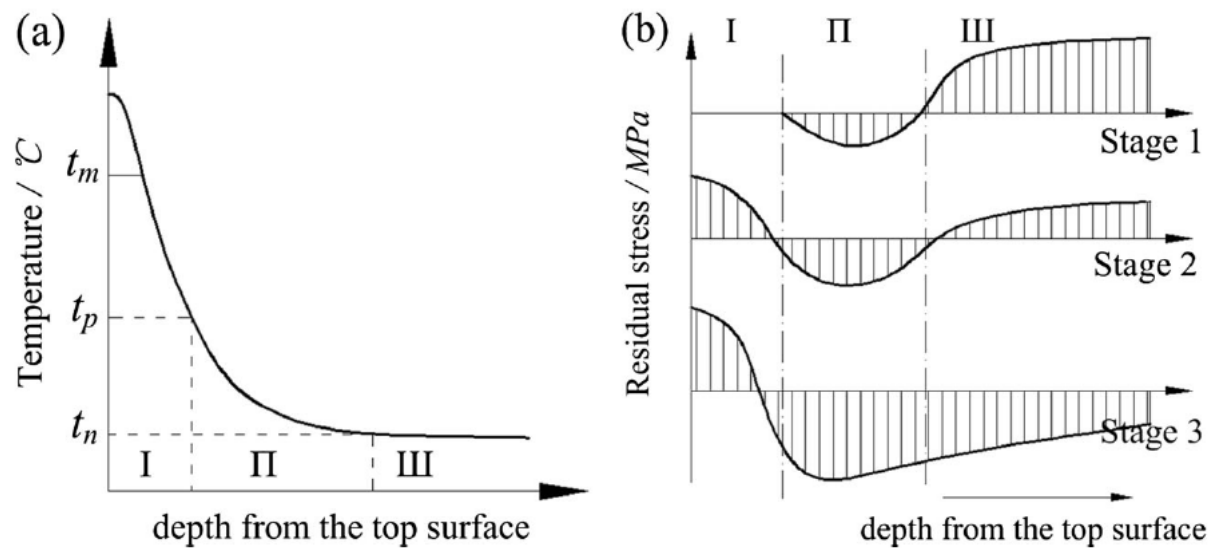

Figure 1.4 - a) Temperature Distribution in SLM in the Build Direction b) Resulting Residual Stress Distribution at Three Stages [33] 
The thermal state of the part can be divided into three regions. The first region is the melted zone, which represents the material that exceeds the plastic temperature during the heating phase. The second region is the heat-affected zone (HAZ) which consists of the material that is raised above ambient temperature, and the third region is the non-affected zone, which stays at ambient temperature [33].

During the heating phase (Stage 1), region I is molten and cannot sustain any residual stresses. Region II experiences the compressive force discussed in the heating phase of the TGM while region III experiences the corresponding tensile force. During the solidification process of the cooling phase (Stage 2), region I drops below $T_{p}$ and contracts. Corresponding to the second residual stress mechanism, the contraction is inhibited by region II resulting in a tensile force in region I and a compressive force in region 2. As the temperature approaches $T_{n}$ (Stage 3) the compressive force from the contraction of region I extends into region III resulting in tensile stress near the surface and compressive stress below [33].

The origin of residual stresses in parts produced by the SLM process has been investigated in numerous studies. Mercelis and Kruth [38] developed a simplified theoretical SLM part stress profile model based on the general beam theory. Figure 1.5 shows general stress profiles predicted by the simplified model of Mercelis and Kruth $[28,38]$. The model was developed to predicted the residual stress profile resulting from the cool-down phase where the upper layer induces stress as a result of thermal contraction; since the stress resulting from the thermal contraction would exceed the yield strength, the tensile stress in each layer is assumed to be equal to the yield strength of the material [28, 38]. According to Mercelis and Kruth [38]:

When a layer is added to the base plate, it induces a compressive stress into the upper part of the base plate and a tensile stress in the lower part. When successive layers are added on top, each layer induces a certain stress profile in the base plate, but also in the underlying solidified layers, thus reducing the initial tensile stress present in these layers. 


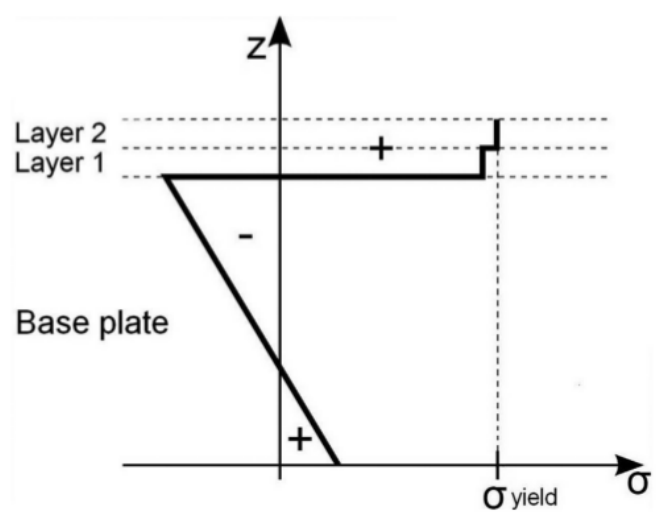

(a)

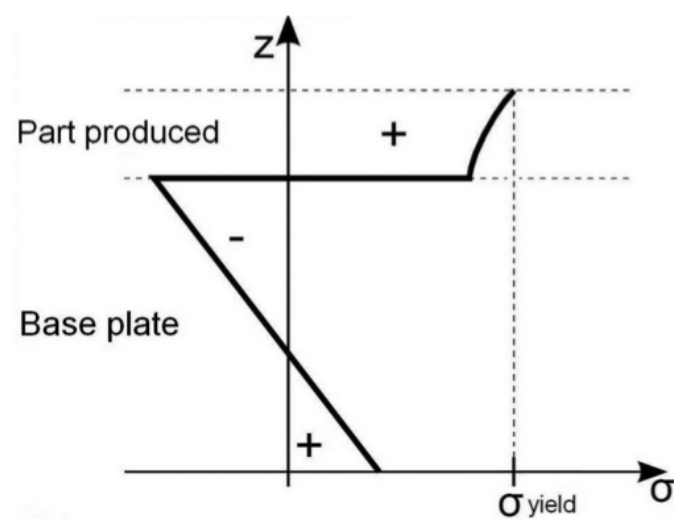

(b)

Figure 1.5 - a) Residual Stress Distribution After Adding Two Layers of Melted Powder b) Residual Stress Distribution After Final Layer [28]

Several other studies have explored the mechanisms of residual stress development in the SLM process including the experimental studies of Knowles et al. [25] and Van Belle et al. [49]; as well as the computational studies of Roberts et al. [43, 44], Matsumoto et al. [36], and Liu et al. [33].

While the mitigation and understanding of residual stresses has been an active area of research since the advent of the SLM process over 20 years ago $[12,40]$, the majority of these studies have focused on simple parts without overhanging features. Of the limited studies that address overhanging features $[40,62]$ most suggest partspecific design guidelines; however, these are only applicable to geometries similar to those in the study. While these rules-of-thumb provide insight into the residual stress issue, they do not provide a detailed understanding of residual stresses development in overhanging features [40].

Support structures are the most common method for addressing the residual stress problem, and several studies deal with the design and optimization of support structures. While support structures are often necessary to build parts with overhang features, they have several undesirable qualities such as increase build time, 
increased material usage, post-processing, and the possibility of damaging the part [4, 24, 40, 42, 62]. Furthermore, the lack of general support structure guidelines results in frequent build failures for complex parts forcing practitioners to use a trialand-error manufacturing process.

The development of general design guidelines for support structures has the potential to greatly reduce the negative factors of support structures and also increase the reliability of the SLM process. To aid in the development of general support structure design guidelines, the present study compared the residual stress distributions in an overhanging feature through a $2^{2}$ factorial study of support structure contact teeth spacing and top length, utilizing a part-scale thermo-mechanical finite element model of the SLM process. 


\title{
CHAPTER 2
}

\author{
Previous Work
}

\subsection{Overview}

This chapter presents a review of the literature related to the study of residual stresses in overhanging features produced with the SLM process. While this review is not a comprehensive evaluation of the existing literature, it is comprised of numerous studies that summarize the findings presented in the literature. For clarity, these studies will be categorized into three major topics, each with several subcategories shown in Figure 2.1.

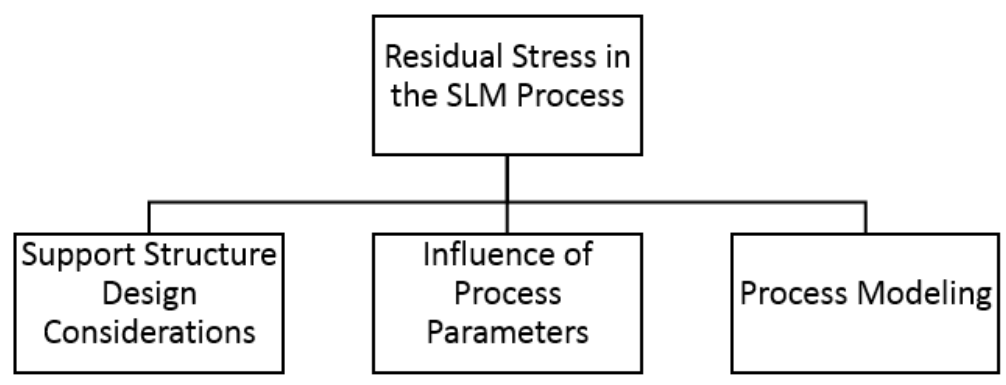

Figure 2.1 - Literature Review Organizational Chart

\subsection{Support Structure Design Considerations}

Support structures are necessary to prevent warping and build failure in many complex SLM parts; however, the use of support structures is inconvenient for practitioners. Parts with supports take longer to build, use more material, require postprocessing, and can occasionally damage the part itself. To reduce the negative effects 
of support structures, several studies have been conducted to optimize support structures. The universal objectives of support structure optimization are reducing the distortion of the part, preventing in-process failures, minimizing support structure material usage, build time, and post-processing time.

According to Calignano [4], support structures can be divided into two functional areas: the contact area, often referred to as teeth, and the main support structure, or support base. Figure 2.2 shows these two distinct regions of the support structure for a simple part with a $90^{\circ}$ overhanging feature. Figure 2.3 is a detailed view of the contact area with several key parameters labeled. For scale, typical tooth heights range from $0.5-2[\mathrm{~mm}]$.

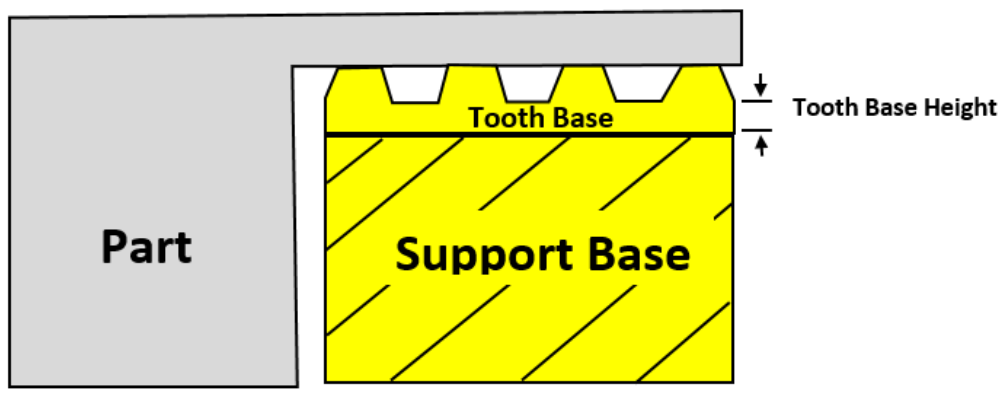

Figure 2.2 - Support Structure Regions 


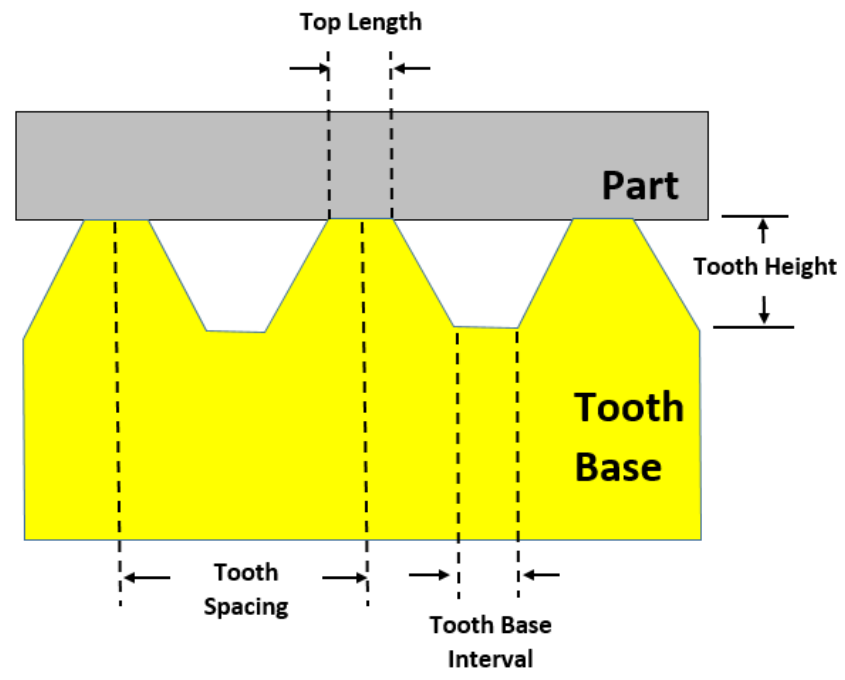

Figure 2.3 - Contact Area Parameters

The research relating to support structure design considerations can be broken into two categories: contact area and main support structure designs.

\subsubsection{Contact Area}

Several studies investigated the effects of varying the contact area parameters. Calignano [4] performed a Taguchi $\mathrm{T}_{36}$ study to investigate the effects of varying six contact area parameters on the warping of simple $20 \times 10 \times 15[\mathrm{~mm}]$ part for both aluminum (AlSi10Mg) and titanium (Ti6Al4V). Calignano [4] found that the tooth height contributed significantly to the warping of both the aluminum and titanium parts. The block hatching was also highly significant for aluminum but it was less significant of a factor for titanium. In both aluminum and titanium Calignano [4] found that the tooth base interval, the $\mathrm{Z}_{\text {offset }}$, and the perforation were not significant to the part warping.

Poyraz et al [42] conducted a factorial study in which the effects of block hatching, fragmentation interval, top length, and the $\mathrm{Z}_{\text {offset }}$ were investigated for a simple part 
with a $90^{\circ}$ overhanging feature shown in Figure 2.4. It is worth noting that in this study, the spacing between the contact points was a function of the block hatching. Poyraz et al. [42] conducted two sets of experiments with Inconel625 (IN625) and found that the smaller fragmentation intervals, block hatching, and by extension the contact spacing resulted in less distortion. Poyraz et al. [42] also discovered that shorter top lengths lead to weaker support attachments.

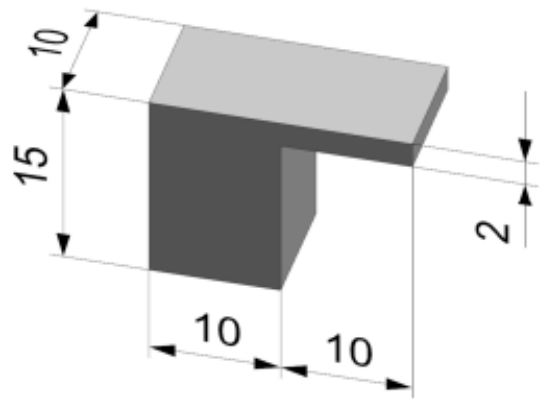

(a)

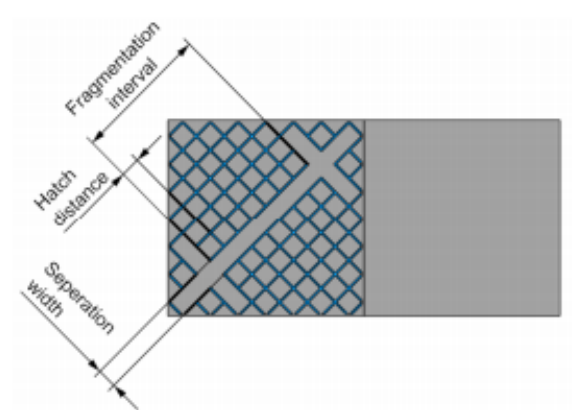

(b)

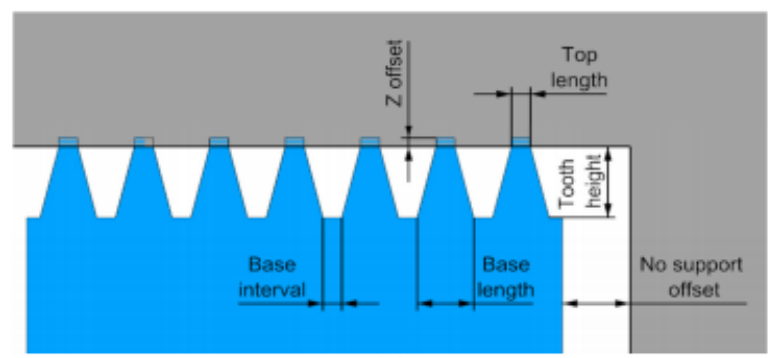

(c)

Figure 2.4 - a) Dimensions of Part b) Block Support Parameters c) Tooth Parameters $[42]$

In addition to studying the effects of several SLM process parameters Liu et al. [33] compared the distortion of the three identical parts shown in Figure 2.5. The support for parts I and II are the same, except for the block hatching, which is 2 [mm] for part I and $1[\mathrm{~mm}]$ for part II. The support for parts I and III are the same except for the top length which is $0.3[\mathrm{~mm}]$ for part I and $1[\mathrm{~mm}]$ for part III. It can be seen in Figure 2.5 that both larger top length and smaller block hatching prevented 
separation between the part and the support structure.

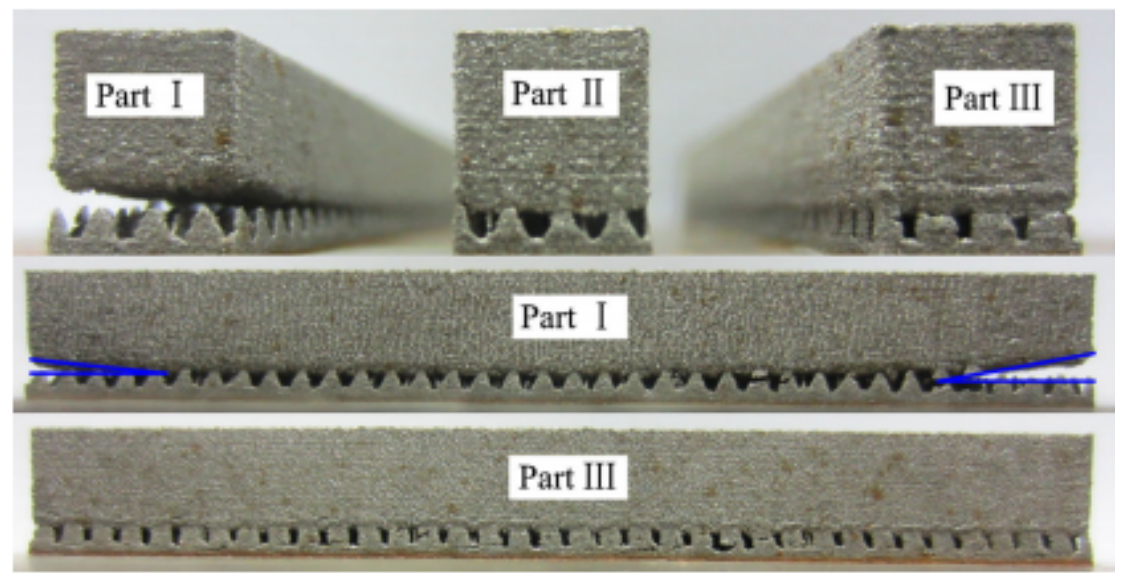

Figure 2.5 - Support Structure Warping Comparison [33]

Gan and Wong [13] did not study supports with the same contact geometry as Poyraz et al. [42]; however, Gan and Wong came to a similar conclusion that decreasing the spacing between contact points decreases the deflection in the final part. In addition to the experimental study, Gan and Wong [13] used a simple 3D finite element method (FEM) model to show the difference between the transient temperature distribution in layers with symmetric and asymmetric support structure contact points shown in Figure 2.6. Gan and Wong [13] found that asymmetric contact points resulted in asymmetric solidification which would produce higher in-plane residual stresses. This prediction agreed well with the experimental data which showed larger deflections in the parts with asymmetric support contact points. 


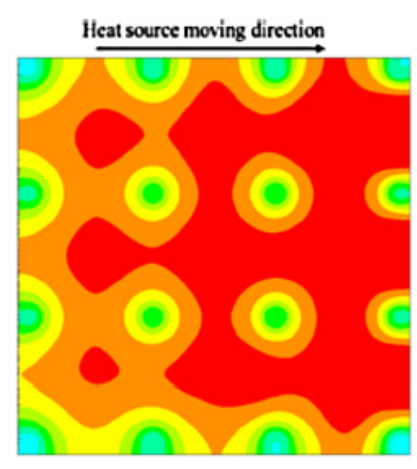

a)

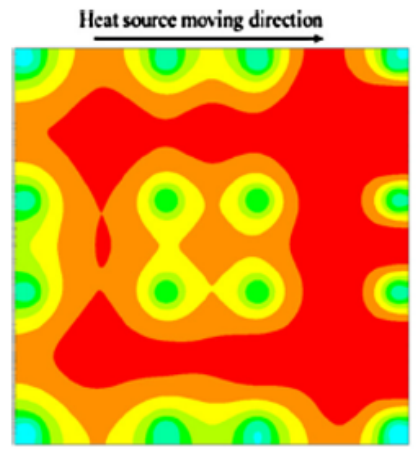

d)

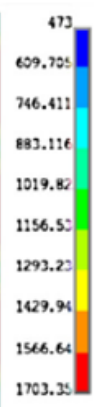

1703.35

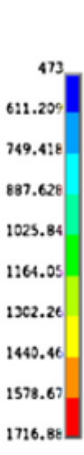

611.205
749.478
887.628
1025.84
1164.05
1302.26
1440.46
1578.67
1716.88

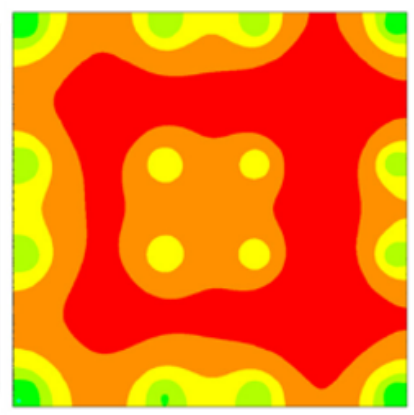

e)

b)
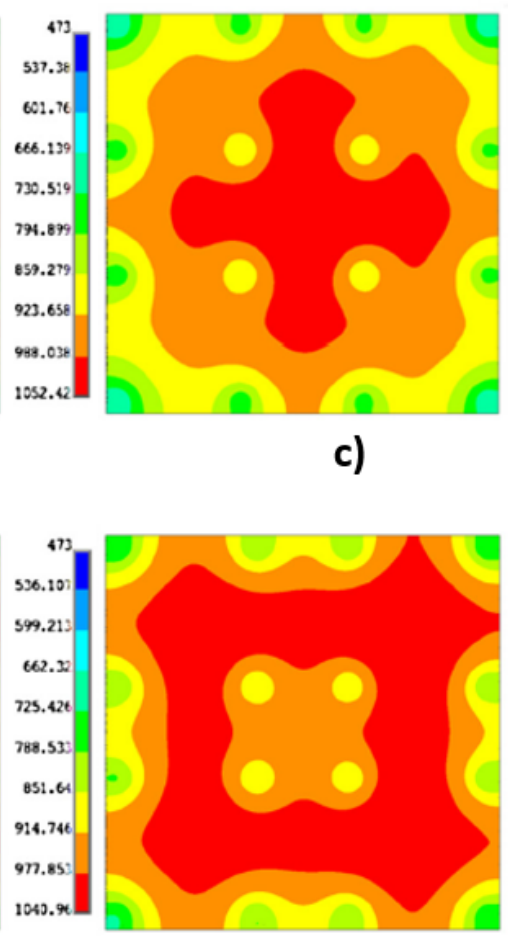

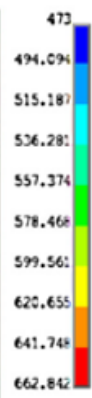

f)

Figure 2.6 - Transient Temperature Distribution in Overhang Layer for Asymmetric Contact Points (a-c) and Symmetric Contact Points (d-f) at 3 Time Intervals [13]

Hussein et al. [22] performed an experimental study to investigate the use of lattice type support structures and found that decreasing the contact point spacing was only advantageous when the volume fraction was increased. Decreasing the contact spacing of the lattice structures is achieved by decreasing the the cell size, which also decreases the size of the contact area (analogous to the top length) for the same volume fraction. Thus, these results do not contradict the results of the other studies mentioned in this section; rather these results only suggest that the interaction between the contact spacing and area are a significant factor for preventing separation between the support and the part. 


\subsubsection{Support Base}

Numerous case studies have compared different support base types with one or more of the universal objectives for support structure optimization in mind. The most notable work in this area is that of Hussein et al. [20, 21] which investigate the use of lattice support structures to minimize the material and build time of the supports while also reducing the post-processing requirements. Hussein et al. [22] found that two types of lattice supports, diamond and gyroid, provide a considerable reduction in material usage and build time while maintaining the structural properties needed to prevent distortion and build failure. It was also noted that there was no obvious distinction between the diamond and gyroid lattice supports for reducing part distortion.

Cloots et al. [8] also investigated the use of lattice type support structures for minimizing material usage and build time. Cloots et al. [8] coupled the design of support structures with a modulated scanning strategy and power input to reduce the structural requirements on the supports. The results of Cloots et al. [8] showed that a segmenting strategy which couples support structure design with modulated process parameters can reduce both the material and build time for supports.

After investigating the distortion of two parts using three different support base types, Gan and Wong [13] concluded that, “...the design of support structures should include three important characteristics: promotes uniform heat dissipation, maximum spacing of $5 \mathrm{~mm}$ between support structures, and the angle bounded by the support structure and the shrinkage direction must be more than $90^{\circ} . "$

Järvinen et al. [24] conducted an experimental study investigating the surface quality of overhanging features and the ease of removal for web and tube type supports with varying characteristic lengths. Järvinen et al. [24] concluded that two support types had comparable surface quality, but the web type support structures had superior ease of removal. 
Bobbio et al. [2] characterized the tensile strength of four different lattice support structures and found that, "...the ligament structural strength was $34-49 \%$ of the strength of fully dense material... due to the stress concentration at the support structure-solid material interfaces..."

One of the fundamental works in computational models of overhanging feature support structures is the single layer 3D thermo-mechanical FEM model of Hussein et al. [21]. In this study, Hussein et al. [21] demonstrated that a consolidated layer built over a powder bed has a lower cooling rate than that of layer built over a solid substrate and the lower cooling rate results in a less stable molten zone.

Cheng and Chou [6] performed a similar study to that of Hussein et al. [21] with a 2D thermo-mechanical FEM model of the electron beam additive manufacturing (EBAM) process and found similar results. In a subsequent study; Cheng and Chou [7] used a similar model to their previous work [6] to study geometric variations in the support structure. Figure 2.7 shows the model setup and Figure 2.8 shows the geometries considered in the study. Several major assumptions were made in simulating the EBAM process, most notably assuming plane strain and an initial uniform temperature distribution in the solid and powder substrates. The details of the setup, including the temperature depend material properties, volumetric heat source, and thermal-mechanical coupling can be found in [7]. 

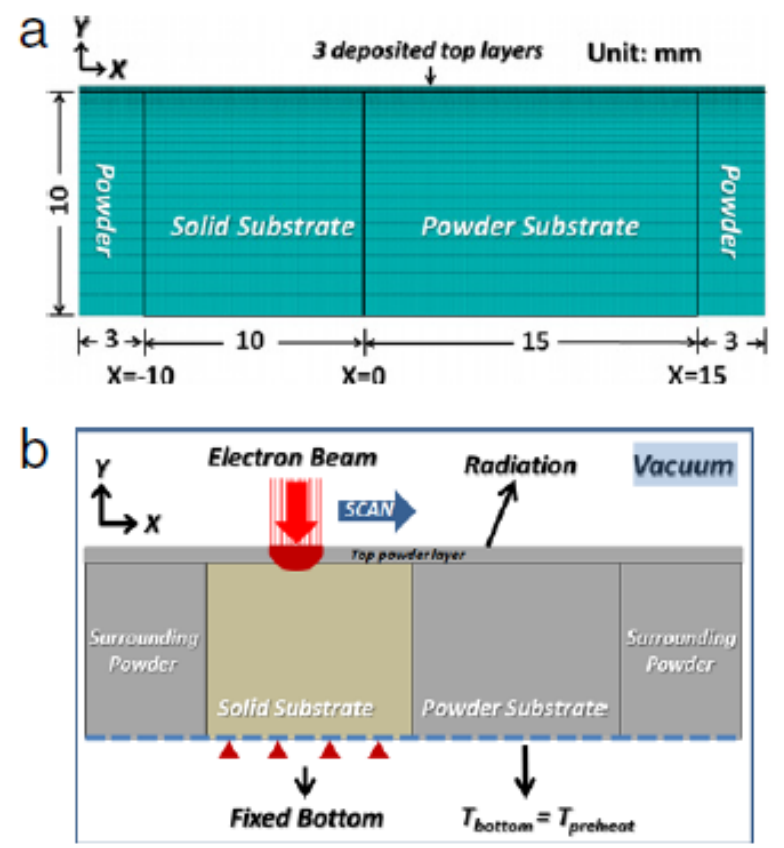

Figure 2.7 - a) Model Geometry b) Model Boundary Conditions [7]

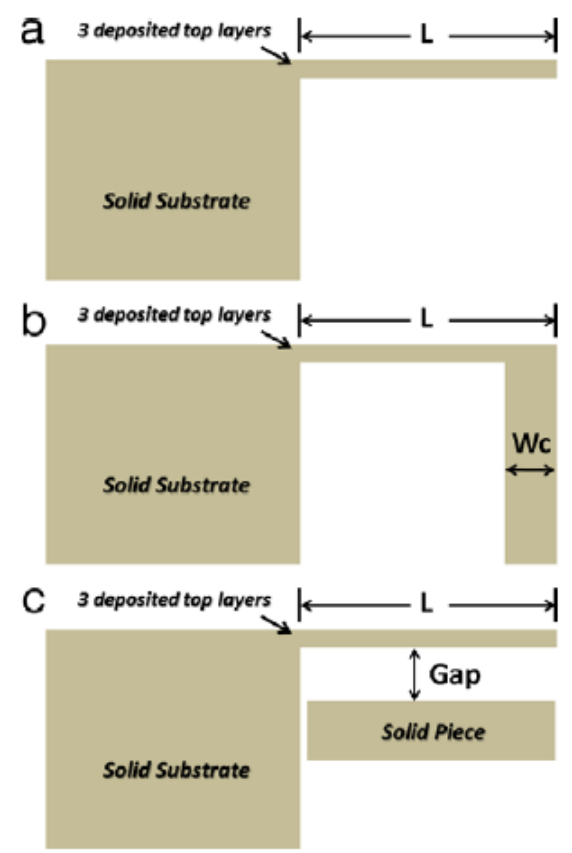

Figure 2.8 - Geometric Variations Considered [7] 
As expected, Cheng and Chou [7] found that increasing the length of the overhang increased the upward deformation at the tip, this is shown in Figure 2.9. Cheng and Chou [7] also found that adding a support to the tip of the overhang decreased the overall deflection; however, the width of the support did not have a large impact on the reduction of the overall deflection, Figure 2.10. The third finding of Cheng and Chou [7] is shown in Figure 2.11, Figure 2.12, and Figure 2.13. The addition of a heat sink near the overhang surface was found to reduce the overall deflection, when the gap was sufficiently small. Figure 2.12 shows that the addition of a heat sink reduced the temperature in the powder bed directly below the overhang, which signifies improved heat dissipation.

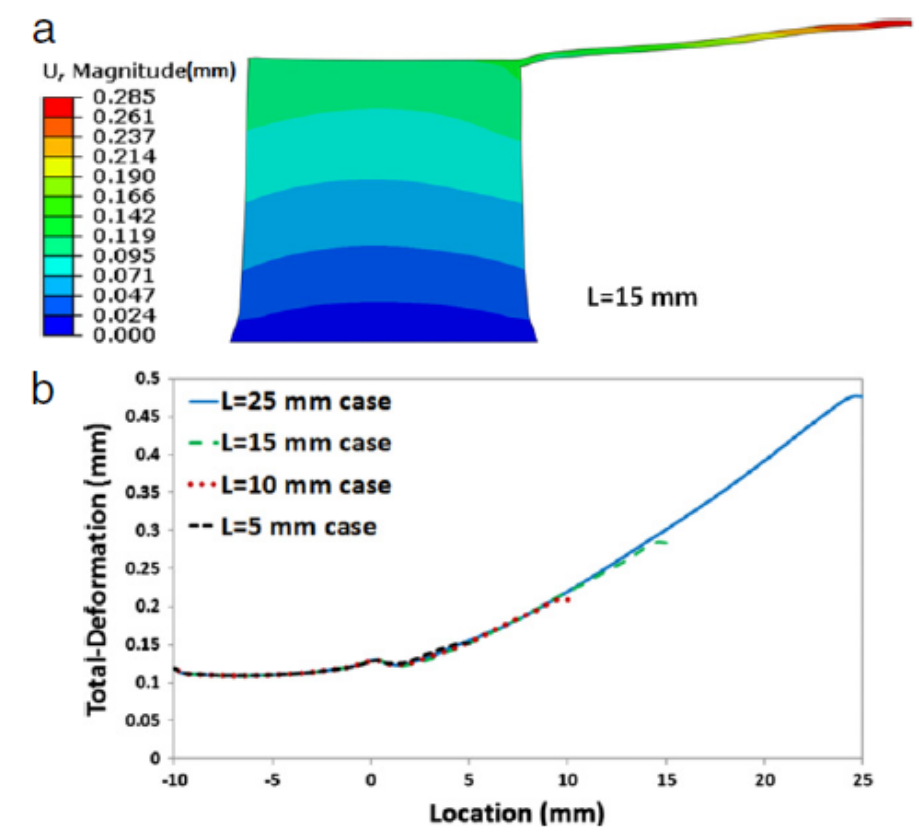

Figure 2.9 - a) Deformation After Cooling $(\mathrm{L}=15[\mathrm{~mm}])$ b)Comparison of Deformations Along Top Surface for Varying Overhang Length [7] 


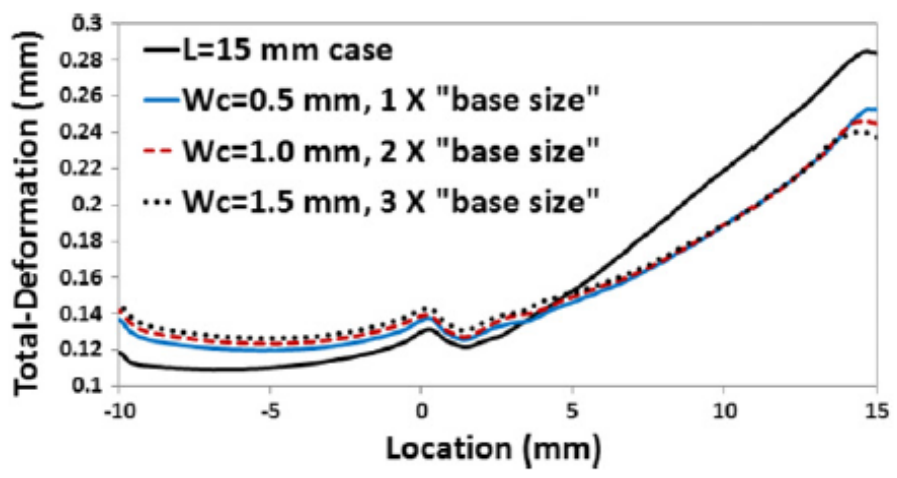

Figure 2.10 - Comparison of Deformations Along Top Surface for Varying Support Width [7]
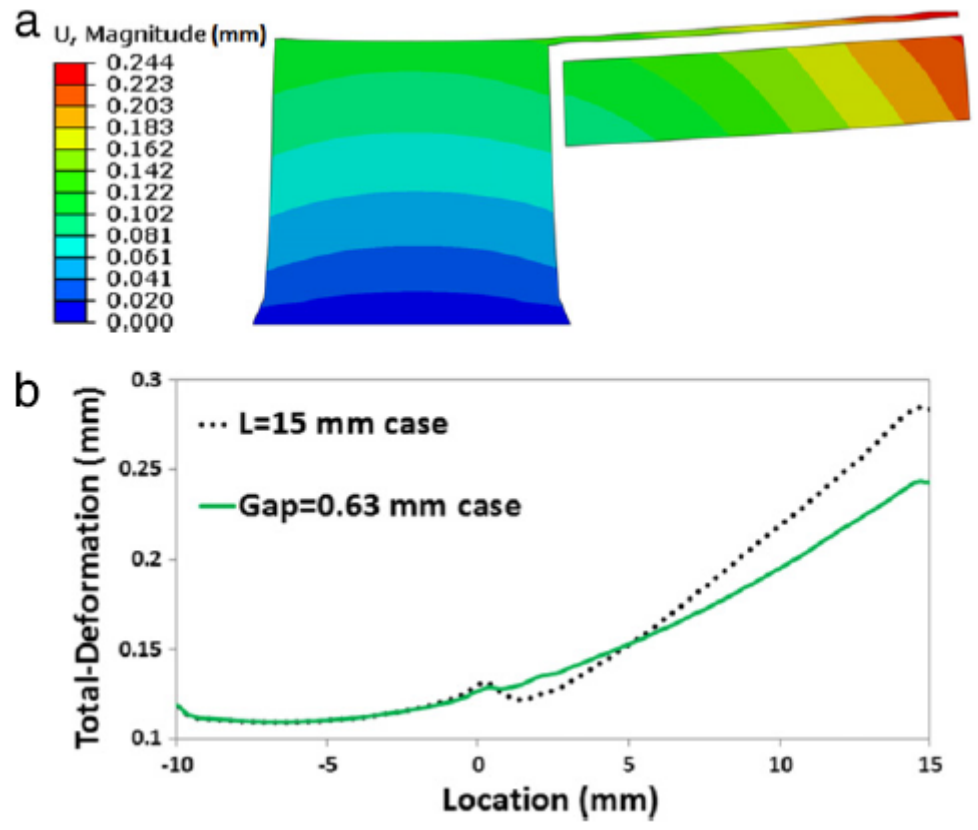

Figure 2.11 - a) Deformation After Cooling (Gap = $0.63[\mathrm{~mm}])$ b)Comparison of Deformations Along Top Surface [7] 


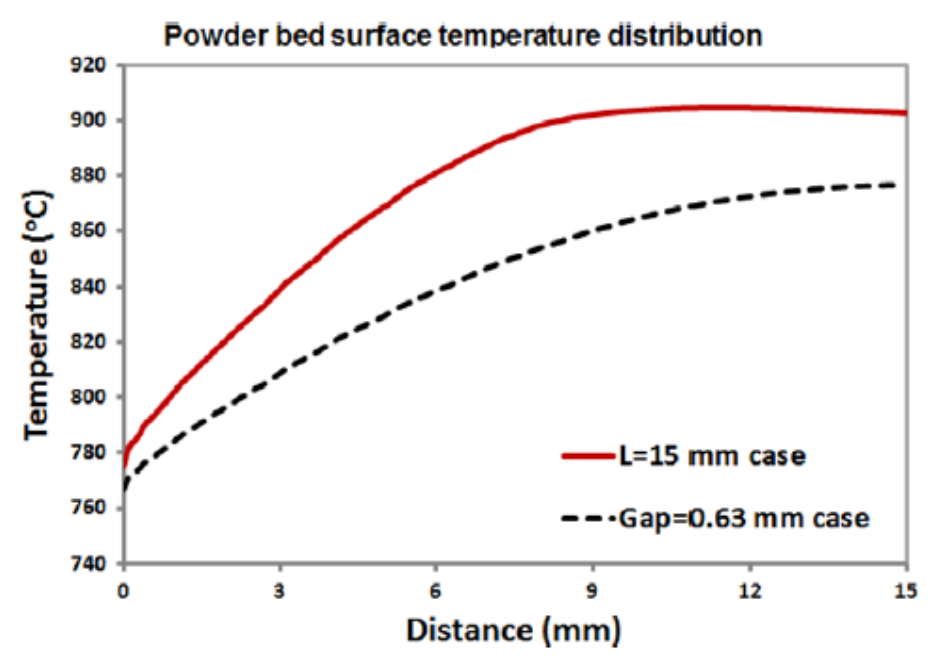

Figure 2.12 - Powder Bed Temperature Distribution Comparison [7]

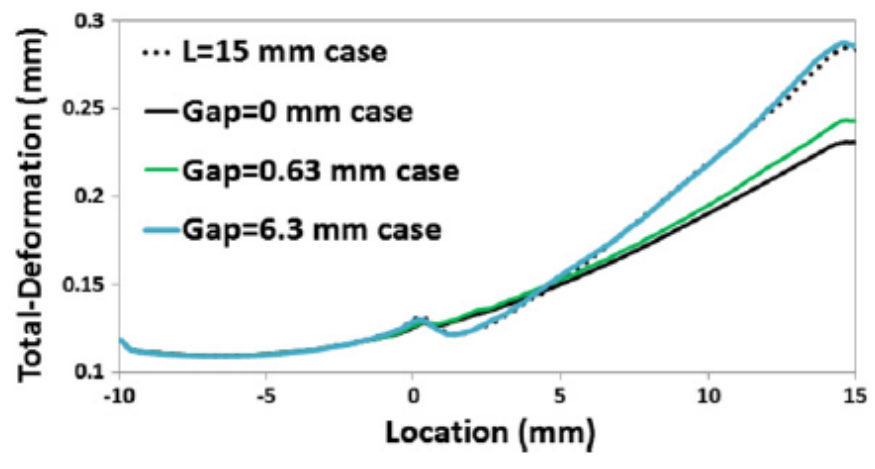

Figure 2.13 - Comparison of Deformations Along Top Surface for Varying Gap Width [7]

The reduction in deflection indicates that improving the heat dissipation alone has a significant impact on the residual stresses in overhanging features. This study by Cheng and Chou [7] has not been experimentally validated; nonetheless, the proposed support structure would require no post-processing so this novel solution merits further investigation.

Vora et al. [51] investigate another solution that would not require any supports physically connected to the part known as anchorless selective laser melting (ASLM). 
The ASLM takes advantage of eutectic alloy systems where the eutectic melting temperature of the alloy is lower than the melting temperature of any pure element in the alloy, see Figure 2.14. In the ASLM process, a powder consisting of two or more pure element particles in the appropriate proportions is melted and forms the alloy in a liquid state. The environmental temperature is held at a temperature below the melting temperature of the pure elements, but above the eutectic melting temperature of the newly formed alloy. Thus, the alloy remains in a semi-solid state and the powder remains solid and unconsolidated [51].

Vora et al. [51] showed that the ASLM process resulted in a residual stress reduction and that the ASLM process could produce complex geometries without support structures; however, this process is limited to a small subset of materials.

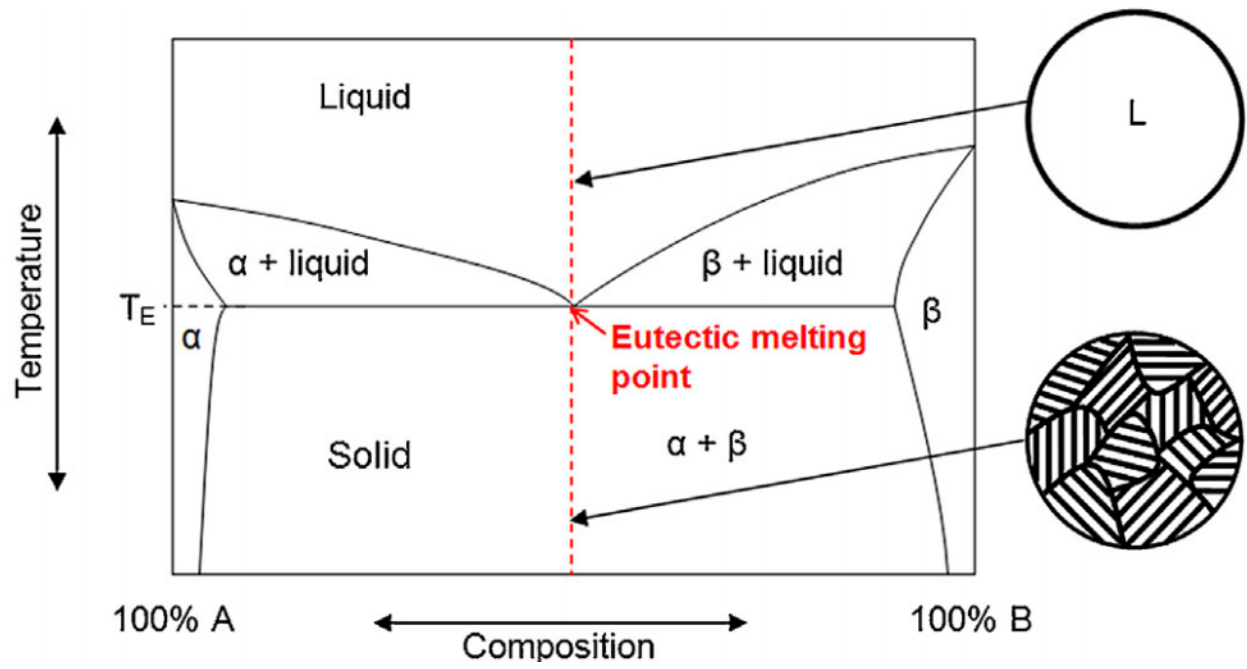

Figure 2.14 - Binary Phase Diagram Containing Material A and B [51]

Jhabvala et al. [23] proposed another innovative approach to support structure design which focused on the build method of the support itself. Jhabvala et al. [23] used a pulsed laser to produce supports with a lower density than the part but were able to resist the deformation of the part and conduct heat to the base plate. 
Jhabvala et al. [23] showed that the pulse laser produced supports were not only easier to remove, but also faster to build than conventional supports.

\subsection{Influence of Process Parameters}

It is well known that process parameters have a large influence of the residual stresses in SLM parts. To-date, most research on optimization of the SLM process has been related to process parameters. Due to the complexity of the SLM process and the coupled effects of various process parameters it is difficult to identify the effects of individual parameters; however, some qualitative conclusions can be reached from a review of the literature. While a typical SLM machine has in excess of 150 process parameters [52], research has been focused on the following: scan speed, scan strategy, laser power, layer thickness, preheating, remelting, and heat treating.

\subsubsection{Scan Speed}

The general consensus among most studies that investigated the effects of scan speed is that lower scan speeds result in lower residual stress accumulation, deformation, and/or cooling rates $[3,21,35,41,47,50,52,54,59]$. Wang et al. [54] and Pohl et al. [41] found contradictory results, where lower scan speeds resulted in higher deformations.

Wang et al. [54] investigate the effects of scan speed, laser power, and scan length on the quality of overhanging features with incline angles ranging from $25^{\circ}$ to $50^{\circ}$ and found that a scan speed of $200[\mathrm{~mm} / \mathrm{s}]$ resulted in more severe warping and more dross formation than a scan speed of $600[\mathrm{~mm} / \mathrm{s}]$ as seen in Figure 2.15. Wang et al. [54] claim that, "lower scanning speed will produce greater inner stress" based on the observed increase in warping; however, neither the residual stress or warping were measured directly. The warping was determined based on visual inspection and the 
point in the process where a "serious defect" occurred that resulted in the process being shut down.

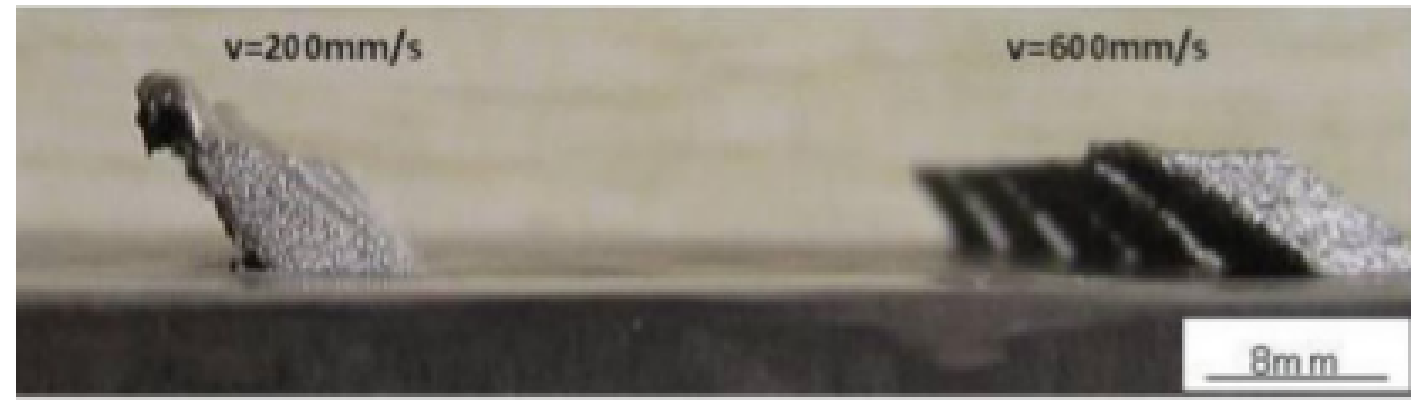

Figure 2.15 - Scan Speed Effect on Warping [54]

Brückner et al. [3] found that lower scan speeds resulted in lower residual stresses using a single track model. Similarly, the single layer model of Hussein et al. [21] showed that Von Mises stresses were reduced when the temperature in the layer was higher and that lower scan speeds increased the layer temperature. According to Manvatkar et al. [35] lower scan speeds reduce cooling rates and Vasinonta et al. [50] showed that it also reduces temperature gradients.

\subsubsection{Scan Strategy}

It has been shown that the scanning strategy is one of the most influential process parameters on residual stresses and deformation $[5,28,41,52,54,58,61]$. There are an inexhaustible number of ways to scan a layer in the SLM process; however, there are several common approaches including zigzag (serpentine), island, strip, helix, or fractal [52]. Across all of these scanning methods it has been seen that shorter scan vectors result in lower residual stresses and/or deformations. It has also been shown that the stress parallel to the scan vector is larger in magnitude than the stress perpendicular to the scan vector [52]. 
Another parameter related to the scan strategy is the downtime, or the time between layers. Shorter downtime between layers allows heat to build up in the part which reduces temperature gradients $[49,52]$; however, this effect varies from material to material [10]. Denlinger et al. [10] showed that shorter downtime reduced the residual stress in Ti6Al4V, but it increased the residual stress in Inconel625.

The laser hatching or the spacing between laser vectors, not to be confused with the block hatching, also effects the deflection in a part. Pohl et al [41] found that increasing the laser spacing decreased the deflection in a sheet of steel.

\subsubsection{Laser Power}

Decreasing the laser power decreases the melt pool temperature and the melt pool size $[12,21,35]$. Lower laser power also decreases warping $[54,58]$ and increases cooling rates [35].In addition to reducing the laser power, the laser mode can be used to decrease the total energy input by pulsing the laser rather than applying a continuous heat input to the powder [8].

In regards to the energy input Wang et al. [54] and Vrancken [52] both made made observations about the relationship between the energy input and the resulting residual stresses and deflections. Wang et al. [54] developed a correlation between energy input $\psi$ and the overhang critical incline angle, shown in Figure 2.16, where larger critical angles correspond to larger deflections. Vrancken [52] discussed the combine parameter effect in terms of the energy density $E$ given by Equation 2.1 where $P$ is the laser power, $v$ is the scan speed, $h$ is the hatching or scan spacing, and $t$ is the layer.

$$
E=\frac{P}{v h t}
$$


Vrancken [52] highlighted that increasing laser power or decreasing laser speed both reduce residual stresses and increase energy density; however, increasing layer thickness also lowers residual stresses and it lowers the energy density. Thus, there does not seem to be a direct correlation between the energy density and the resulting residual stresses.

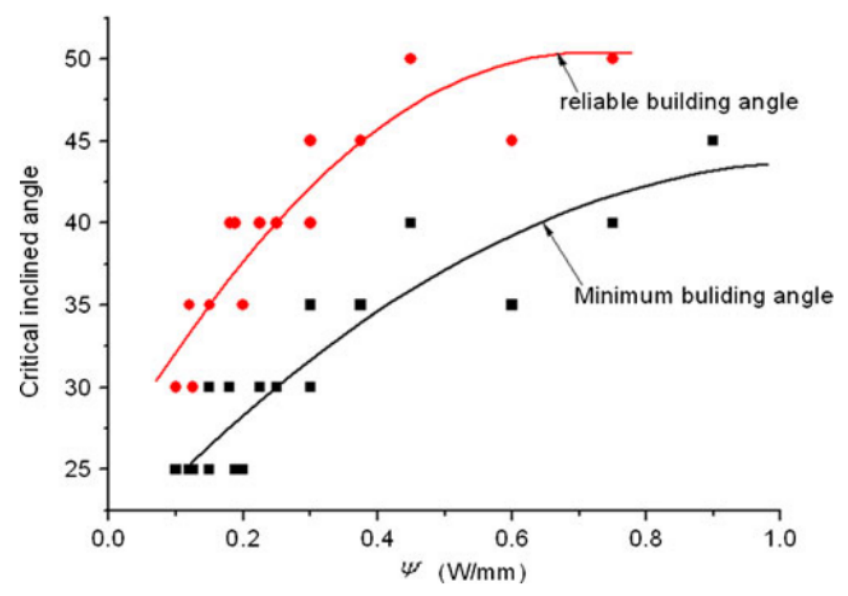

Figure 2.16 - Critical Incline Angle and Energy Input Correlation [54]

\subsubsection{Layer Thickness}

The three different part geometries studied in [5, 28, 49] all exhibited reduced deformation as a result of increasing layer thickness.

\subsubsection{Preheating}

According to Vrancken [52], "It is unanimously agreed upon by all literature sources that preheating reduces residual stresses." Studies such as [3, 5, 28, 45, 50, 61] all confirm the observation that preheating the powder by heating the base plate reduces the residual stress. Vrancken [52] suggests that while preheating does reduce 
temperature gradients, the main mechanism for reducing the residual stresses is the reduction of yield stress in the part due to the elevated temperatures.

\subsubsection{Remelting}

Another heating method that has been shown to effectively reduce residual stresses is remelting of previously solidified layers [45, 60]. Shiomi et al. [45] showed that remelting layers resulted in a 55\% improvement in residual stresses compared to a $40 \%$ improvement using preheating of the base plate.

\subsubsection{Post-Process Heat Treating}

Compared to preheating and remelting that can reduce residual stresses by as much as $40 \%$ and $55 \%$, respectively, heat treating a part produced my SLM can reduce the residual stresses by as much as 70\% [45]. Vrancken [52], Kruth et al. [28], and Carter et al. [5] also saw a reduction in residual stresses using a post-process heat treatment.

\subsubsection{Process Parameter Summary}

As summarized by Vrancken [52]:

Residual stresses, deformations, temperature gradients, and/or cooling rates are reduced by:

1. A higher laser power,

2. Low scan speed,

3. Thicker layers,

4. Shorter scan vectors,

5. Use of preheating.

\subsection{Process Modeling}

As Patterson et al. [40] states, "...a good model is needed for problem understanding before any useful work on the problem can be attempted." Due to the complexity 
of the SLM process the majority of modeling attempts have been focused on specific aspects of the process, while only a few general process models exist. Therefore a review of these modeling approaches is best present in the three categories: thermal models, thermo-mechanical, and material models.

\subsubsection{Thermal Models}

While the analytical or semi-analytical techniques used in $[11,30,32]$ provide useful insight into the SLM process, they have many limitations [63]. Since analytical and semi-analytical models cannot capture the complexity of the thermal problem in the SLM process, most research has utilized FEM [40, 63].

Contuzzi et al. [9], Kundakcioglu et al. [29], and Huang et al. [19] all produced models which simulate the temperature distribution in the SLM process [40]. One of the most notable studies is the one developed by Gusarov et al. [15] which estimated an effective volmetric heat source and included radiation. Roberts et al [44, 43] modeled the temperature distribution in a multi-layer SLM process using a simulation technique known as "element birth and death." Fu and Guo [12] also modeled the temperature distribution in multi-layer SLM process.

For additional sources, a tabulated comparison of existing numerical studies that focus on the thermal modeling of the SLM process can be found in [63].

\subsubsection{Thermo-Mechanical Models}

Extensive research $[28,38,39,48,52,53]$ in the stress and distortion of parts produced by the SLM process has been conducted at the University of Leuven [40]. One of the most noteworthy works in the field [38], which presented a simple analytical model used to predict the stress caused by the cool-down phase. 
Contuzzi et al. [9], Hussein et al. [21], and Matsumoto et al. [36] all investigated the stress and deformation in the SLM process using single layer thermo-mechanical models. Several studies have also investigated the stress using multi-layer models including $[17,31,61]$. Each of the multi-layer studies used an equivalent heat source to drastically reduce the computational cost by combining the heat input of $20-100$ $[\mu m]$ layers into several larger layers, typically on the order of $1[\mathrm{~mm}]$. As Zaeh and Branner stated:

To account for the numerous physical effects within the manufacturing process, different strategies are fundamental for an industrially useful simulation. Due to the available computing power, for example, it is not always feasible to model every single scanning vector within the energy application.... Contrary to a more specified layer model, which considers the exact scanning strategy, it is the aim of the global model to substitute the scanning vectors of every layer by scanning areas. Thus it is possible to calculate the residual stresses and deformations of the whole part. A global model based on whole parts contains most of the occurring transient physical effects within the manufacturing process, e.g. comparable temperature gradients, melting and re-melting, a repeated change of the material properties or metallurgical phase transformations. Only the exact scanning strategy is disregarded.

Zaeh and Branner's [61] global model applied a 200 [W] thermal load for 20 [ms] over each $1[\mathrm{~mm}]$ layer. Li et al. [31] reduced the part into seven zones shown in Figure 2.17. The elements in the zones were activated, heated, and cooled in a sequential order. A detailed description of the effective scan strategy used by Hodge et al. $[16,17]$ will be presented in Chapter 3 .
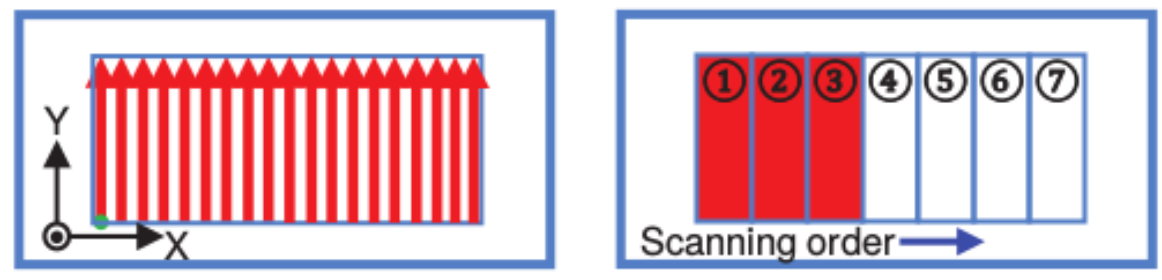

Figure 2.17 - Global Model Scan Strategy [31] 


\subsubsection{Material Models}

Both the development of residual stresses during the SLM process and the materials response to residual stresses are dependent on material properties. The influence of thermal and mechanical properties are valid for all metal alloys; however, an alloys response to residual stresses can be specific to that particular alloy or a subset of alloys [52]. If no allotropic transformations or other phenomena occur; thermal diffusivity, thermal expansion coefficients, yield stress, Youngs modulus, and melting point all influence the magnitude of the resulting residual stresses [52].

Since the material properties play a critical role in modeling residual stresses, determining appropriate material models is critical. Fortunately there is a general consensus in the literature on material models. Regardless of the bulk material [7, $12,16,17,21]$ all material models used temperature-dependent material properties.

For the part scale models of the SLM process which included support structures $[26,61]$, the supports were modeled as simplified geometries with material properties correlated to match the properties of the real supports. Zeng [62] proved the validity of this approach for thermal conductivity and Zhang et al. [64] validated the use of effective material properties for cellular structures produced with AM. 


\title{
CHAPTER 3
}

\author{
Model Development
}

\subsection{Overview}

This chapter outlines the development and implementation of part-scale thermomechanical finite element models used to simulate the SLM process. Four numerical simulations were conducted to investigate the residual stresses induced during the SLM process and to study the effect of varying contact area parameters. The models are based on the work of the Lawrence Livermore National Laboratory (LLNL) Methods Development Group (MDG) and were implemented in Diablo, a multi-physics finite element code developed by LLNL.

The multi-physics model, developed and validated by Hodge et al. [16, 17], couples a thermal model with a mechanical model using a staggered coupling scheme. The thermal model is comprised of the balance of thermal energy and phase change while the solid mechanics model consists of the balance of mass, linear momentum, and angular momentum $[16,17]$.

The values of the Tooth Spacing and the Top Length for each of the four simulations are shown in Table 3.1. Visual representations of Tooth Spacing and the Top Length can be seen in Figure 2.3 and a side view of Test 4 is shown in Figure 3.1. 


\begin{tabular}{|c|c|c|}
\hline Test & Tooth Spacing $[\mathrm{mm}]$ & Top Length $[\mathrm{mm}]$ \\
\hline 1 & 0.5 & 0.15 \\
\hline 2 & 1.0 & 0.15 \\
\hline 3 & 0.5 & 0.35 \\
\hline 4 & 1.0 & 0.35 \\
\hline
\end{tabular}

Table 3.1 - Factorial Study Design Points

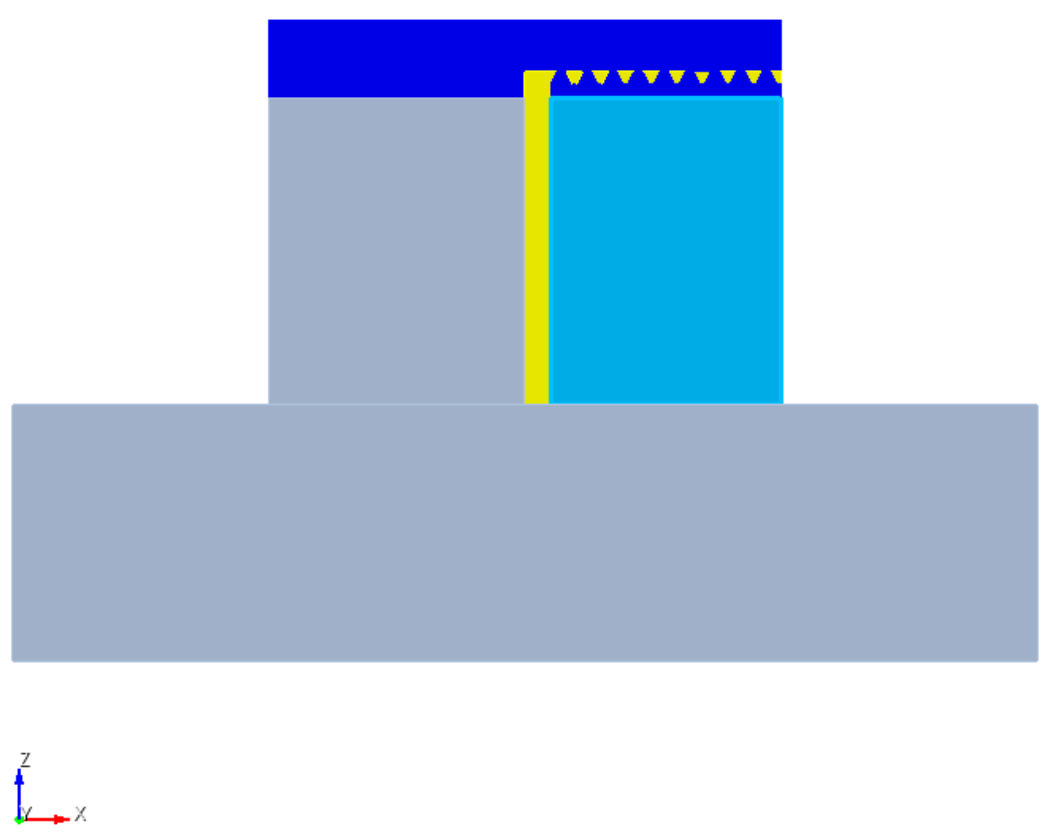

Figure 3.1 - Side View of Test 4

Each model consisted of a 40x30x10 [mm] base plate, a 20x10x15 [mm] part, and a 9x10x13 [mm] support structure under the overhanging feature. To reduce the computational cost, the first $12[\mathrm{~mm}]$ of the part and the support structure were modeled as consolidated substrate at the onset of the simulation with a nonuniform initial temperature distribution. The displacement of the bottom and sides of the 
base plate were fixed in the direction normal to each surface, and the initial stress was zero throughout the model. The bottom of the base plate was also assigned a fixed temperature of $303[\mathrm{~K}]$.

Four distinct material models were used and are represented by the dark blue, light blue, grey, and yellow regions in Figure 3.1. The grey region represents the base plate and the first $12[\mathrm{~mm}]$ of the part. The light blue region represents the first 12 [mm] of the support structure. The yellow region represents unconsolidated powder that is not consolidated during the simulation, and the dark blue region represents unconsolidated powder that is consolidated.

\subsection{Thermal Model}

Two distinct physical phenomena are represented in the thermal model $[16,17]$. The first is the balance of thermal energy and is described by the strong form of the governing equation for the temperature, $T$, and the associated boundary conditions:

$$
\begin{aligned}
\rho c_{p} \frac{\partial T}{\partial t} & =-\operatorname{div}(\mathbf{q})+r, \quad \text { in } \quad \Omega \\
T\left(\mathbf{x}_{\mathbf{T}}, t\right) & =\bar{T}, \quad \text { on } \quad \mathbf{x}_{\mathbf{T}} \in \Gamma_{T} \\
\mathbf{q}\left(\mathbf{x}_{\mathbf{q}}, t\right) & =\overline{\mathbf{q}}, \quad \text { on } \quad \mathbf{x}_{\mathbf{q}} \in \Gamma_{q} \\
T(\mathbf{x}, 0) & =T_{0}, \quad \text { on } \quad \Omega \cup \partial \Omega
\end{aligned}
$$

where $\rho$ is the density, $c_{p}$ is the specific heat, $t$ is the time, $r$ is a volumetric heat source, and $\mathbf{q}$ is the heat flux. $\Gamma_{T}$ and $\Gamma_{q}$ are sections of the domain boundary that correspond to the essential and natural boundary conditions, respectively. $T_{0}$ is the initial condition over the union of the domain, $\Omega$, and the boundary, $\partial \Omega$ [16]. 
The phase change model is given by the Stefan-Neumann equation:

$$
\begin{aligned}
T\left(\mathbf{x}_{\mathbf{p}}, t\right) & =T_{p}, \quad \text { on } \quad \mathbf{x}_{\mathbf{p}} \in \Gamma_{p} \\
\left(\mathbf{k}_{\mathbf{1}} \frac{\partial T_{1}}{\partial \mathbf{x}}-\mathbf{k}_{\mathbf{2}} \frac{\partial T_{2}}{\partial \mathbf{x}}\right) \bullet \mathbf{n} & =H \rho \frac{\partial \mathbf{x}_{\mathbf{p}}}{\partial t} \bullet \mathbf{n}, \quad \text { in } \quad \Omega
\end{aligned}
$$

where the phase change occurs along a internal surface, $\Gamma_{p}[16,18]$. The vector, $\mathbf{n}$, is the normal to the surface $\Gamma_{p}$ and the subscripts $(\bullet)_{1}$ and $(\bullet)_{2}$ correspond to the material to either side of the surface $\Gamma_{p}$. The thermal conductivity and the latent heat associated with the phase change are given by $\mathbf{k}$ and $H$, respectively $[16,18]$.

In [16] the heat input from the laser is modeled as a volumetric heat source using the model described by Gusarov et al. [15] given in Equations 3.7-3.9.

$$
\begin{aligned}
r\left(\xi_{1}, \xi_{2}, \xi_{3}\right) & =-\beta_{h} Q_{0} \frac{\partial u}{\partial \xi_{3}} \\
Q_{0} & =\hat{Q}\left(Q_{e}, r_{h}, R\right) \\
u & =\hat{u}\left(L, \rho_{h}, \exp \left(\xi_{3}\right)\right)
\end{aligned}
$$

where $\xi_{i}$ are the coordinates of the moving reference frame of the laser, $\beta_{h}$ is the extinction coefficient, $u$ is the intensity distribution, $r_{h}^{2}=\xi_{1}^{2}+\xi_{2}^{2}, R$ is the laser beam radius, $L$ is the layer depth, $\rho_{h}$ is the hemispherical reflectively of the powder, and $Q_{e}$ is the effective source magnitude that is scaled to account for heat rejection mechanism such as radiation and evaporation [16]. Figure 3.2 shows the laser radiation transfer in a powder layer where $z$ is the same as $\xi_{3}$ in the equations above and $I$ is the radiation intensity, similar to $u$. 


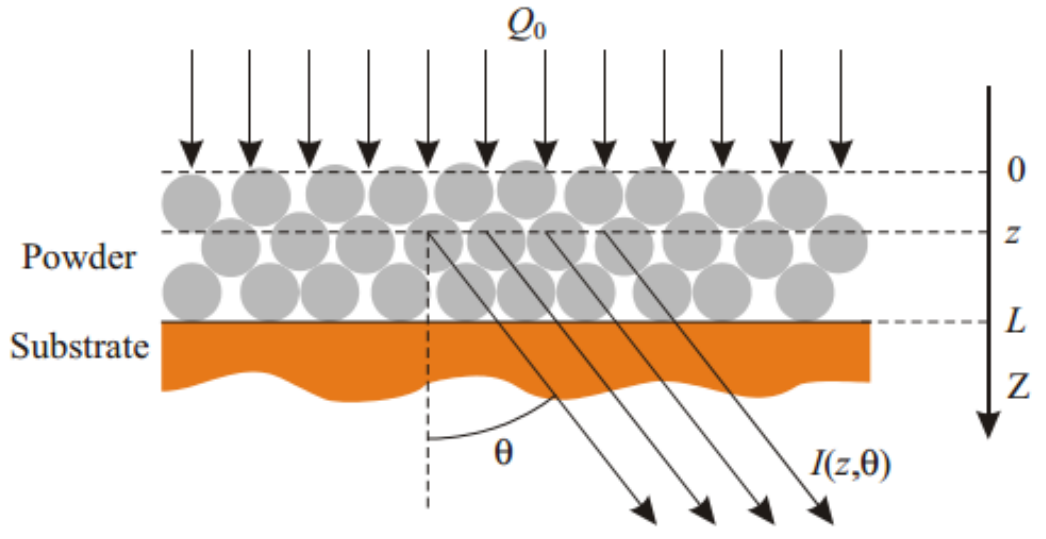

Figure 3.2 - Laser Radiation Transfer in a Powder Layer on a Substrate [15]

In the present study, a uniform heat source was used which is a simplification of the Gusarov et al. [15] model that assumed a constant intensity over the entire laser radius and depth. The uniform model is shown in Figure 3.3 and the volumetric heat source is given by:

$$
r=\hat{r}\left(Q_{e}, R, L, \rho_{h}, \beta_{h}\right)
$$

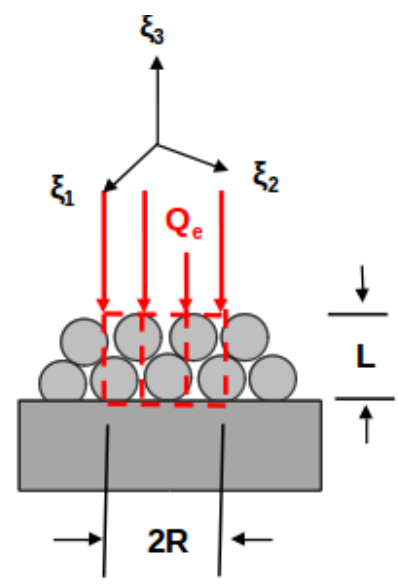

Figure 3.3 - Uniform Laser Heat Source Model 


\subsection{Thermo-Mechanical Model}

The solid mechanics model is based on satisfying the balance of mass, linear momentum, and angular momentum [16]. The solution to the balance of angular momentum results in the constraint that the Cauchy stress tensor, $\sigma$, must be symmetric, $\sigma=\sigma^{\mathbf{T}}$. The balance of mass and angular momentum and corresponding boundary conditions are described by:

$$
\begin{aligned}
\dot{\rho} & =(\mathbf{v}), \quad \text { in } \quad \Omega \\
\rho \dot{v} & =(\sigma)+\rho \mathbf{b}, \quad \text { in } \quad \Omega \\
\mathbf{u}\left(\mathbf{x}_{\mathbf{T}}, t\right) & =\overline{\mathbf{u}}, \quad \text { on } \quad \mathbf{x}_{\mathbf{u}} \in \Gamma_{u} \\
\mathbf{t}\left(\mathbf{x}_{\mathbf{T}}, t\right) & =\overline{\mathbf{t}}, \quad \text { on } \quad \mathbf{x}_{\mathbf{t}} \in \Gamma_{t} \\
\rho(\mathbf{x}, 0) & =\rho_{0}, \quad \text { on } \quad \Omega \cup \partial \Omega \\
\mathbf{u}(\mathbf{x}, 0) & =\mathbf{u}_{0}, \quad \text { on } \quad \Omega \cup \partial \Omega \\
\mathbf{t}(\mathbf{x}, 0) & =\mathbf{t}_{0}, \quad \text { on } \quad \Omega \cup \partial \Omega
\end{aligned}
$$

where $\rho$ is the density, $\mathbf{v}$ is the linear velocity, $\mathbf{u}$ is the displacement, the vector $\mathbf{t}$ is a surface traction, and the scalar $t$ is time. Similarly to the governing equations for the thermal model, $\Gamma_{u}$ and $\Gamma_{t}$ are sections of the domain boundary that correspond to the essential and natural boundary conditions, respectively [16].

Due to the coupling of the thermal and solid mechanics, solving for the Cauchy stress is not trivial. Hodge et al. [16] describe several mechanisms that couple the thermal and mechanical problems; however, the extent of the coupling is best demonstrated through inspection of the constitutive relationships discussed in Section 3.4. 


\subsection{Material Models}

Four material models were used to simulate different phases and properties of $316 \mathrm{~L}$ stainless-steel. The four materials are shown in Figure 3.4 where the grey, dark blue, light blue, and yellow regions correspond to material 1,2,3, and 4 respectively.

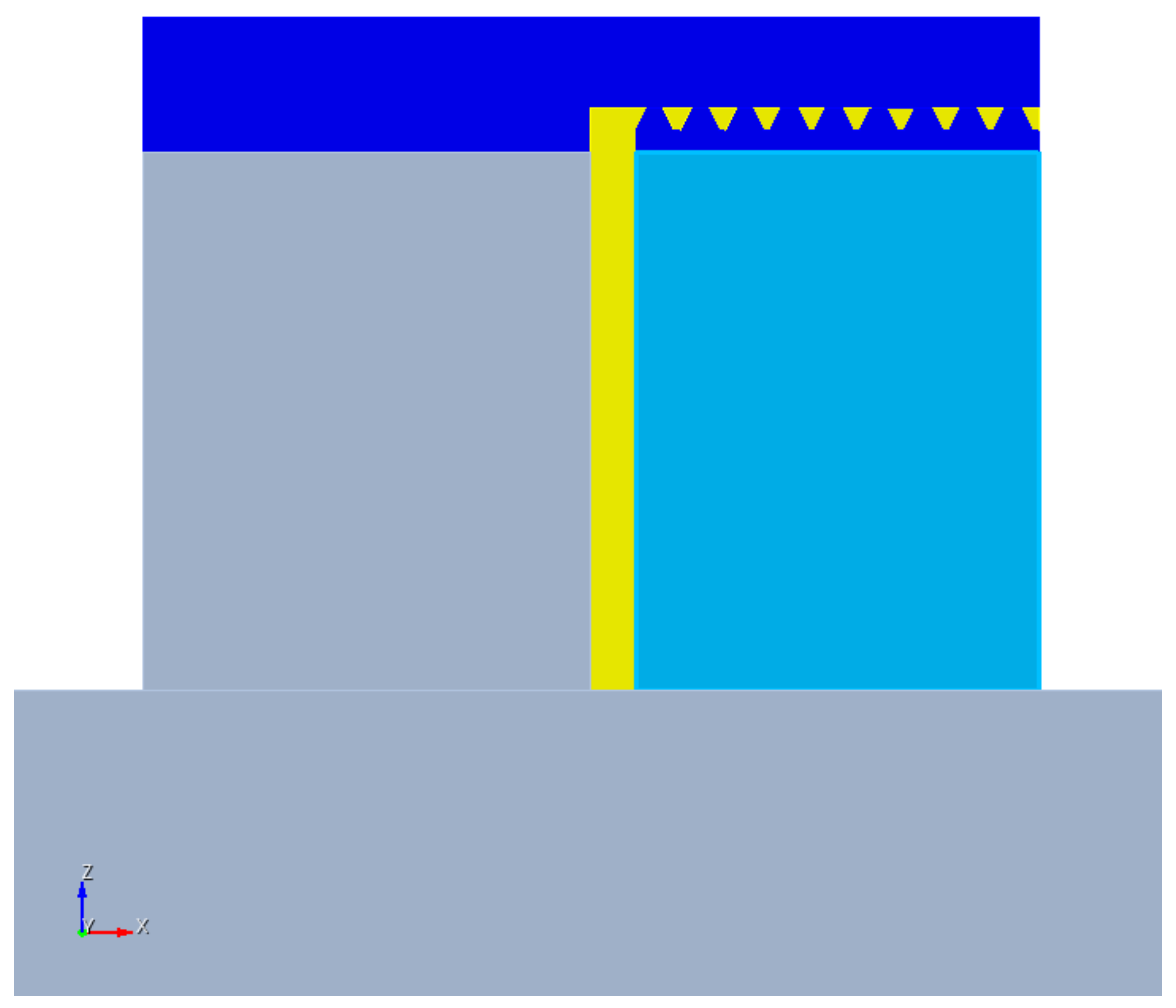

Figure 3.4 - Side View of Test 4

\subsubsection{Material Model 1}

The thermal model for material 1 is isotropic conduction with temperature-dependent properties and phase change [46]. The mechanics model for material 1 is isotropic elastic-plastic material with temperature-dependent properties and isotropic hardening. The stress constitutive equation: for material 1 is given by: 


$$
\begin{aligned}
\hat{\sigma}_{\mathbf{n}+\mathbf{1}} & =\hat{\sigma}_{\mathbf{n}}+\Delta \mathbf{D}: \epsilon_{n, \text { elastic }}+\mathbf{D}_{n+\frac{1}{2}}:\left(\Delta \epsilon-\Delta \epsilon_{\text {thermal }}-\Delta \epsilon_{\text {plastic }}\right) \\
\Delta \mathbf{D} & =\left.\frac{\partial \mathbf{D}}{\partial E}\right|_{n+\frac{1}{2}}\left(E_{T_{n+1}}-E_{T_{n}}\right)+\left.\frac{\partial \mathbf{D}}{\partial \nu}\right|_{n+\frac{1}{2}}\left(\nu_{T_{n+1}}-\nu_{T_{n}}\right) \\
\epsilon_{\text {thermal }} & =\alpha_{T}\left(T-T_{\text {ref }}\right) \mathbf{I} \\
\epsilon_{n, \text { elastic }} & =\mathbf{D}^{-1}: \hat{\sigma}_{n}
\end{aligned}
$$

where, $E$ is the Young's Modulus, $T_{r e f}$ is the reference temperature where there is no thermal strain, and $\alpha_{T}$ is the secant coefficient of thermal expansion [46]. The subcript $(\bullet)_{n+1 / 2}$ denotes quantities calculated at time, $t_{n+1 / 2}$ to account for temperature dependence. $\mathbf{D}$ is a fourth order tensor of material properties defined such that:

$$
\sigma^{+}=\mathbf{D}: \mathbf{d}
$$

where $\sigma^{+}$is an objective Cauchy stress rate and $\mathbf{d}$ is the rate-of-deformation tensor $[16,46]$.

Figures 3.5 - 3.10 show the temperature dependent material properties for material 1. The material data was taken from [14]. Note, the change in material properties at $T=1700[\mathrm{~K}]$ accounts for the phase change from solid to liquid since phase, $\phi$, is used to describe the transition from the powder phase to the consolidated phase. 


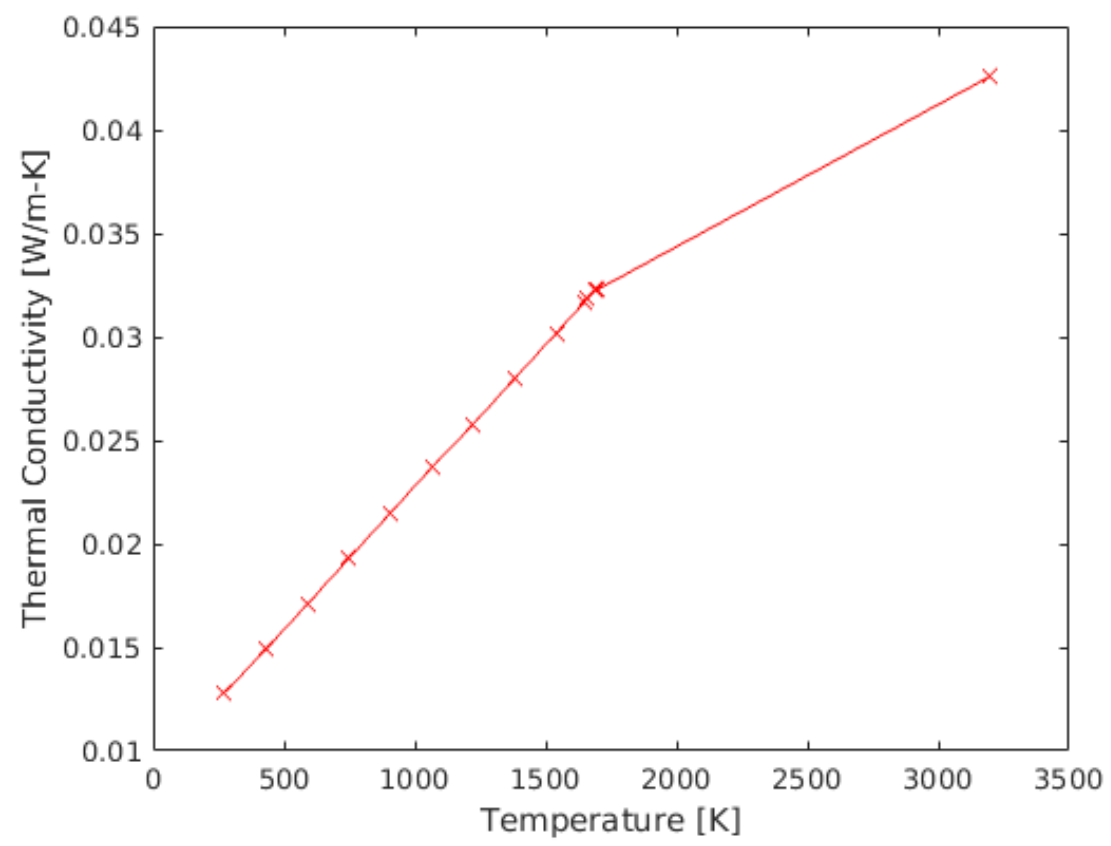

Figure 3.5 - Material 1 Temperature Dependent Thermal Conductivity [14]

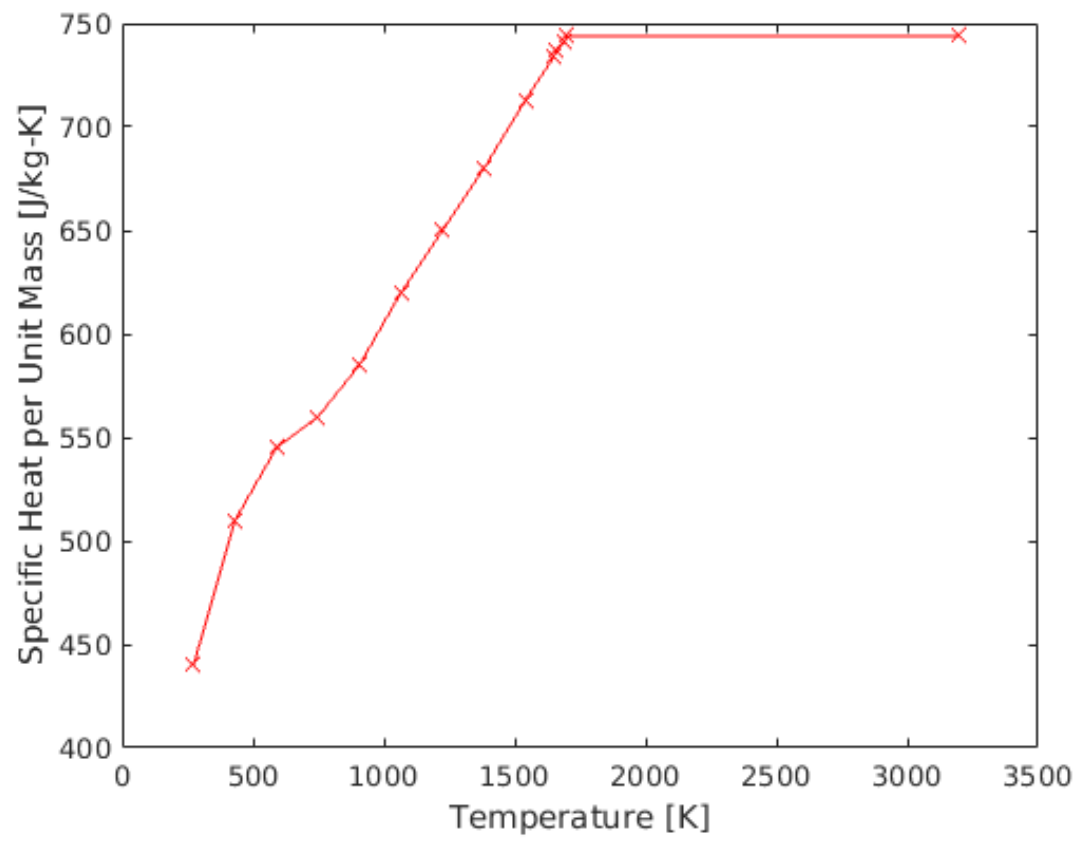

Figure 3.6 - Material 1 Temperature Dependent Specific Heat [14] 


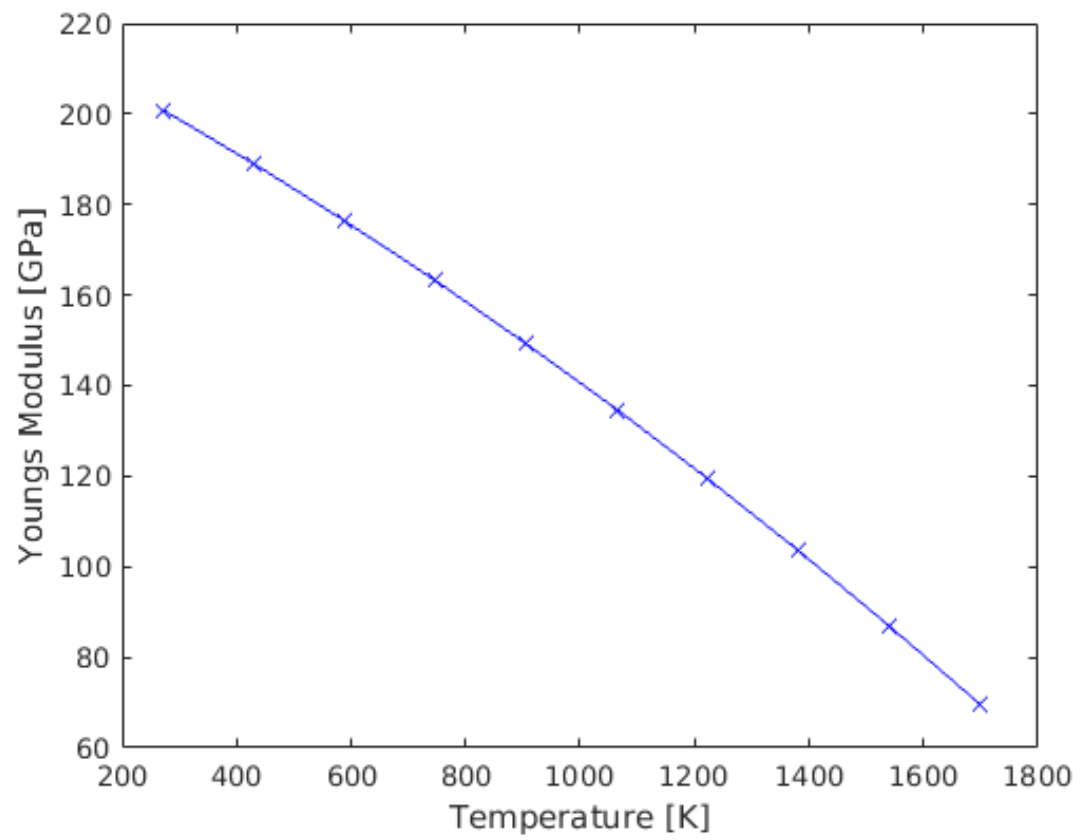

Figure 3.7 - Material 1 Temperature Dependent Young's Modulus [14]

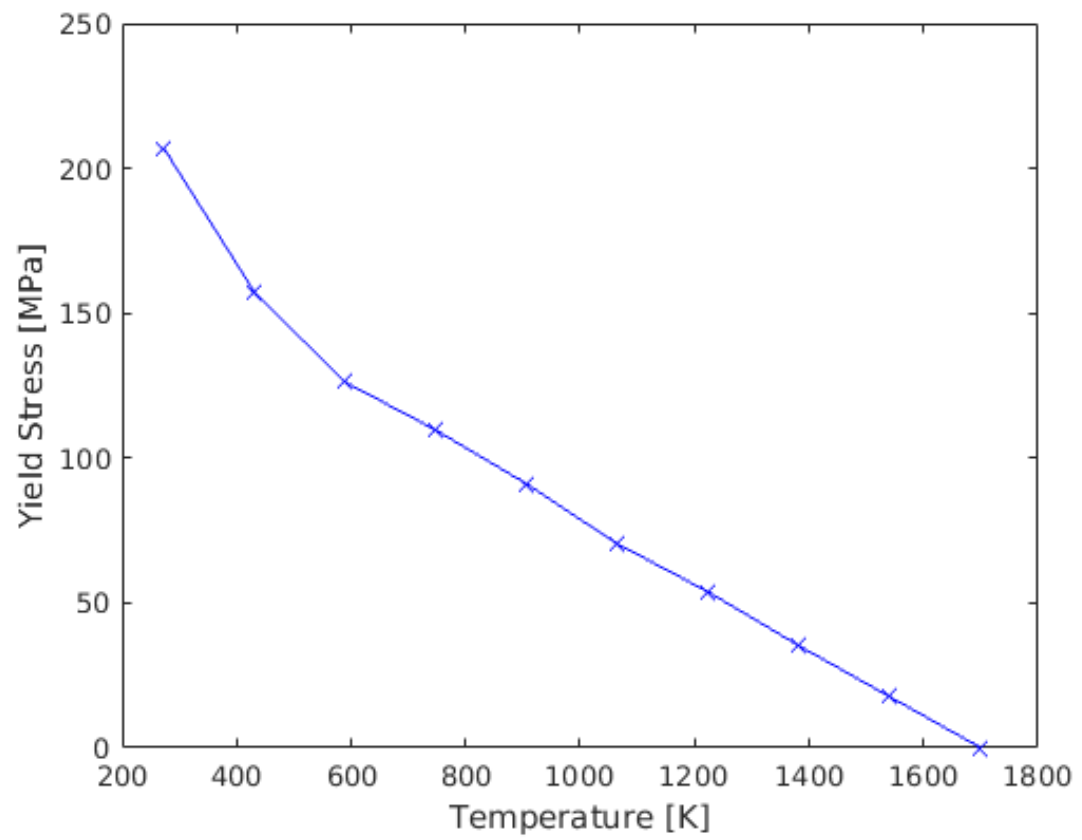

Figure 3.8 - Material 1 Temperature Dependent Yield Stress [14] 


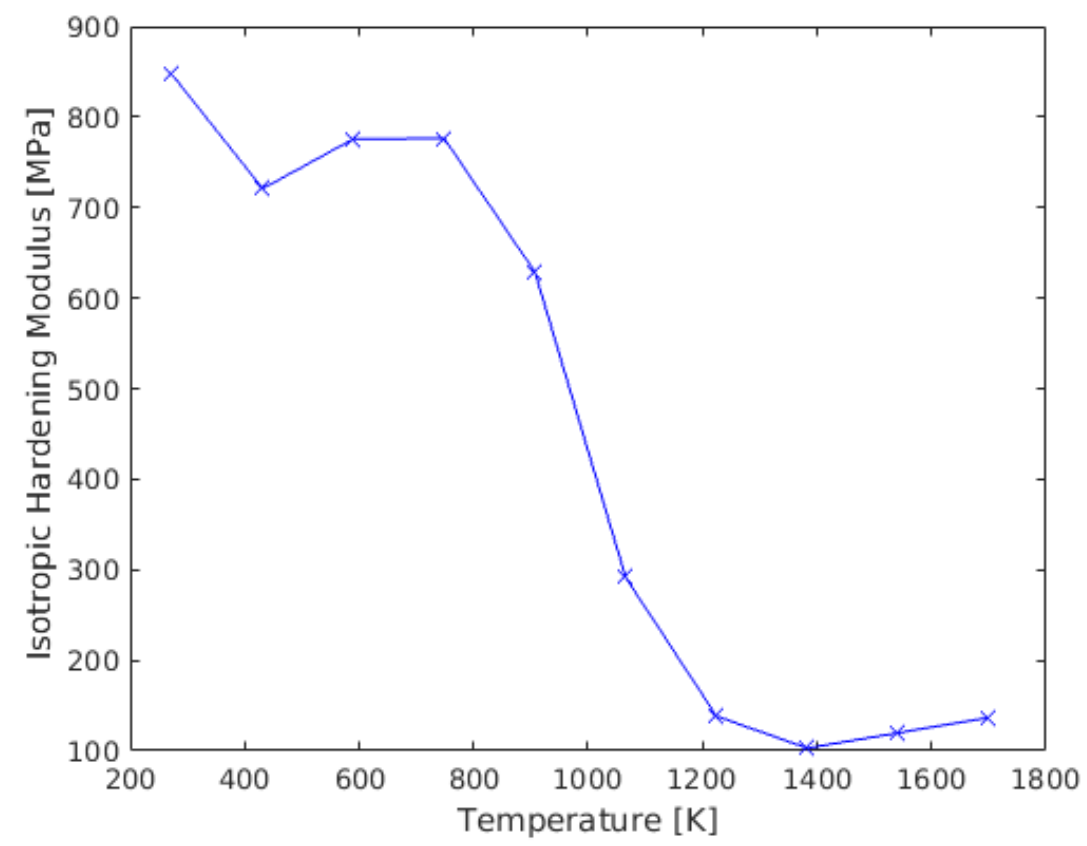

Figure 3.9 - Material 1 Temperature Dependent Isotropic Hardening Modulus [14]

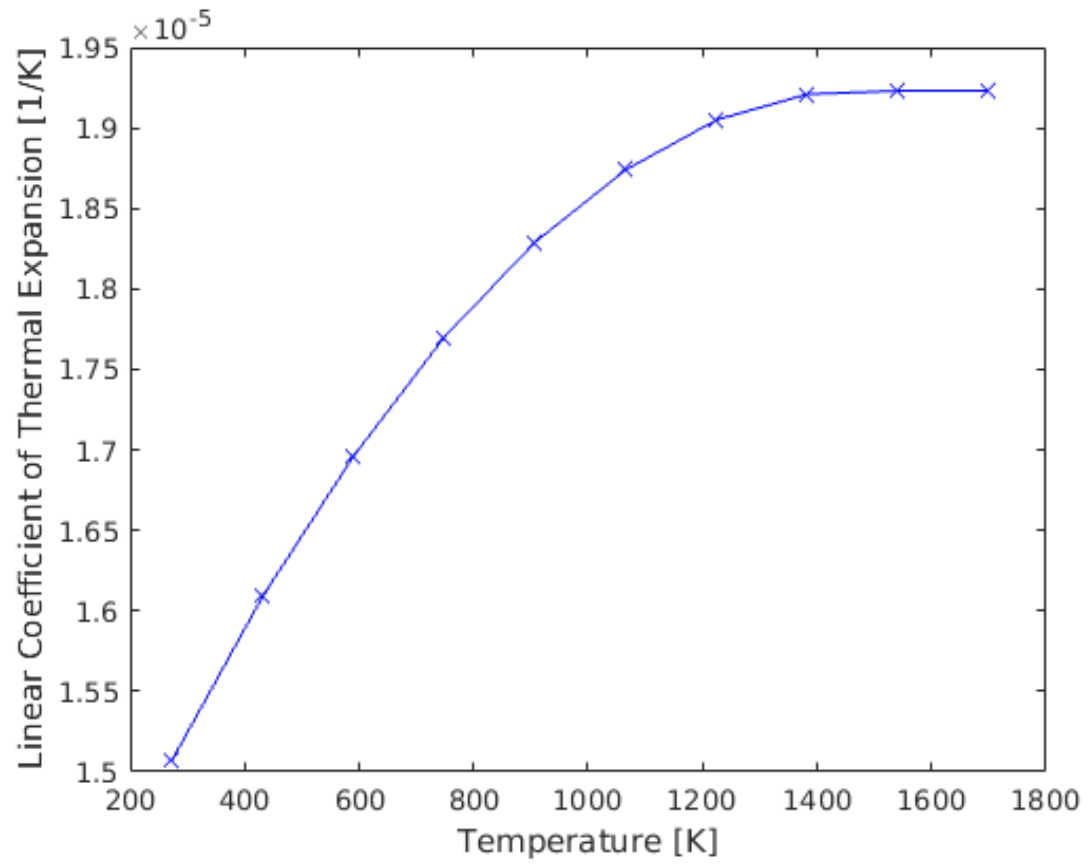

Figure 3.10 - Material 1 Temperature Dependent Coefficient of Thermal Expansion [14] 


\subsubsection{Material Model 2}

Material 2 is the same as material 1, except it accounts for the phase change from the powder phase to the consolidated phase. Accounting for phase change from powder to consolidate material results in the following stress constitutive equation [46]:

$$
\begin{aligned}
\hat{\sigma}_{\mathbf{n}+\mathbf{1}} & =\hat{\sigma}_{\mathbf{n}}+\Delta \mathbf{D}: \epsilon_{n, \text { elastic }}+\mathbf{D}_{n+\frac{1}{2}}:\left(\Delta \epsilon-\Delta \epsilon_{\text {thermal }}-\Delta \epsilon_{\text {phase }}-\Delta \epsilon_{\text {plastic }}\right) \\
\Delta \mathbf{D} & =\left.\frac{\partial \mathbf{D}}{\partial E}\right|_{n+\frac{1}{2}}\left(E_{T_{n+1}}-E_{T_{n}}\right)+\left.\frac{\partial \mathbf{D}}{\partial \nu}\right|_{n+\frac{1}{2}}\left(\nu_{T_{n+1}}-\nu_{T_{n}}\right) \\
\epsilon_{\text {thermal }} & =\left(\phi_{1} \alpha_{1}\left(T-T_{\text {ref } 1}\right)+\phi_{2} \alpha_{2}\left(T-T_{\text {ref } 2}\right)\right) \mathbf{I} \\
\epsilon_{\text {phase }} & =\left(\phi_{1} \beta_{1}+\phi_{2} \beta_{2}\right) \mathbf{I} \\
\epsilon_{n, \text { elastic }} & =\mathbf{D}^{-1}: \hat{\sigma}_{n}
\end{aligned}
$$

Figures 3.11 - 3.16 show the temperature dependent material properties for both the consolidated phase and the powder phase of material 2. Once the powder temperature exceeds $1700[\mathrm{~K}]$, the powder becomes consolidate material therefore the material properties for the powder are truncated at $T=1700[\mathrm{~K}]$ in the following figures. It is worth noting that the thermal conductivity of the powder increases considerably as the temperature reaches approximately $1690[\mathrm{~K}]$ due to increased contact between the particles. 


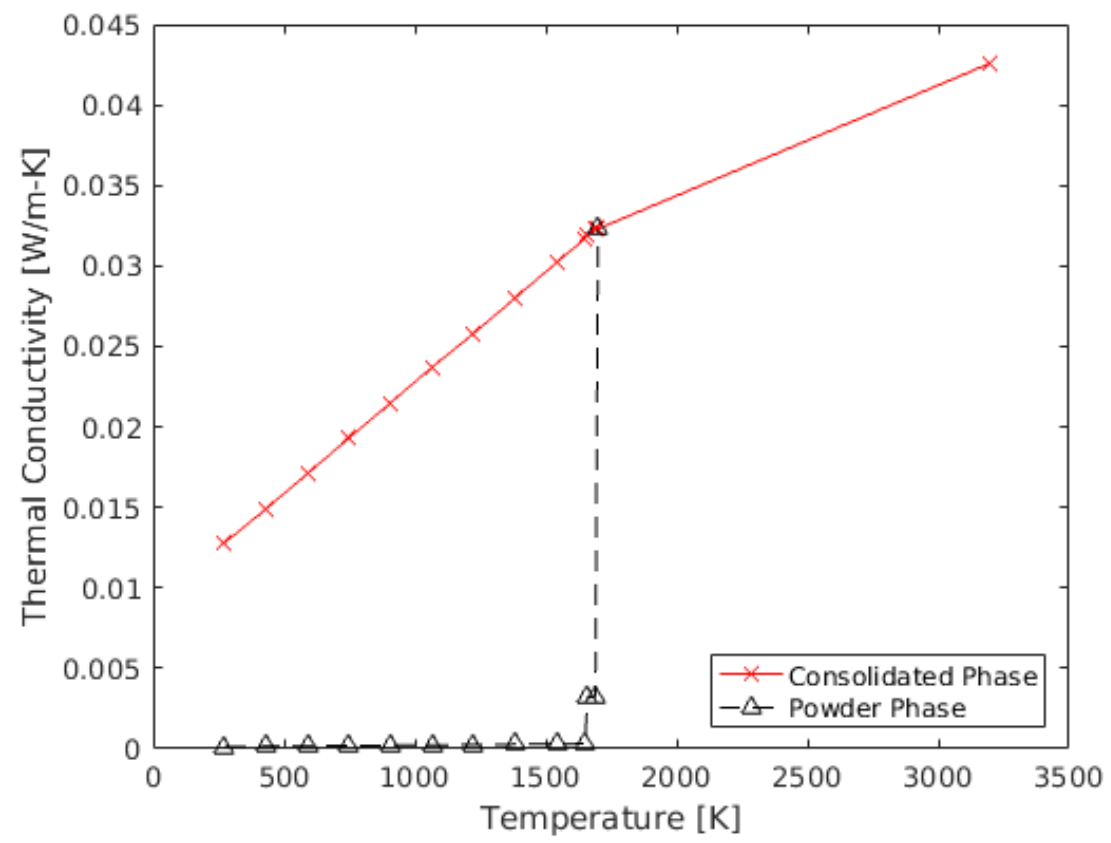

Figure 3.11 - Material 2 Temperature Dependent Thermal Conductivity [14]

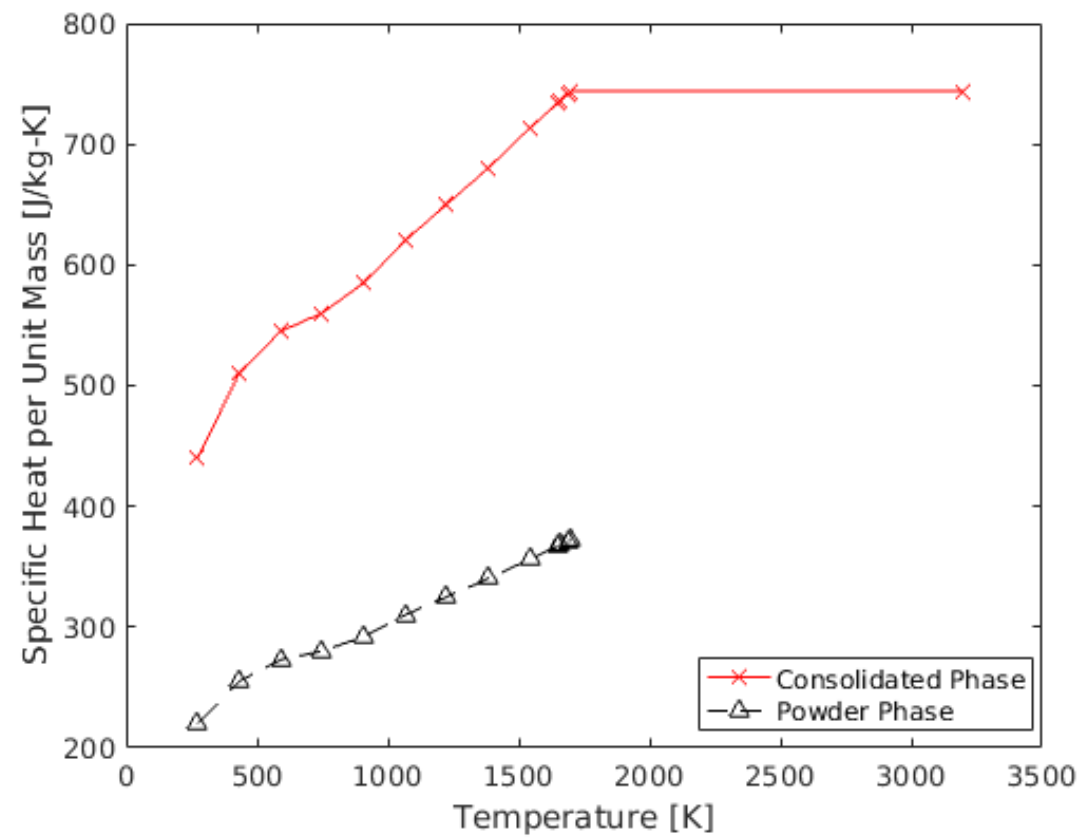

Figure 3.12 - Material 2 Temperature Dependent Specific Heat [14] 


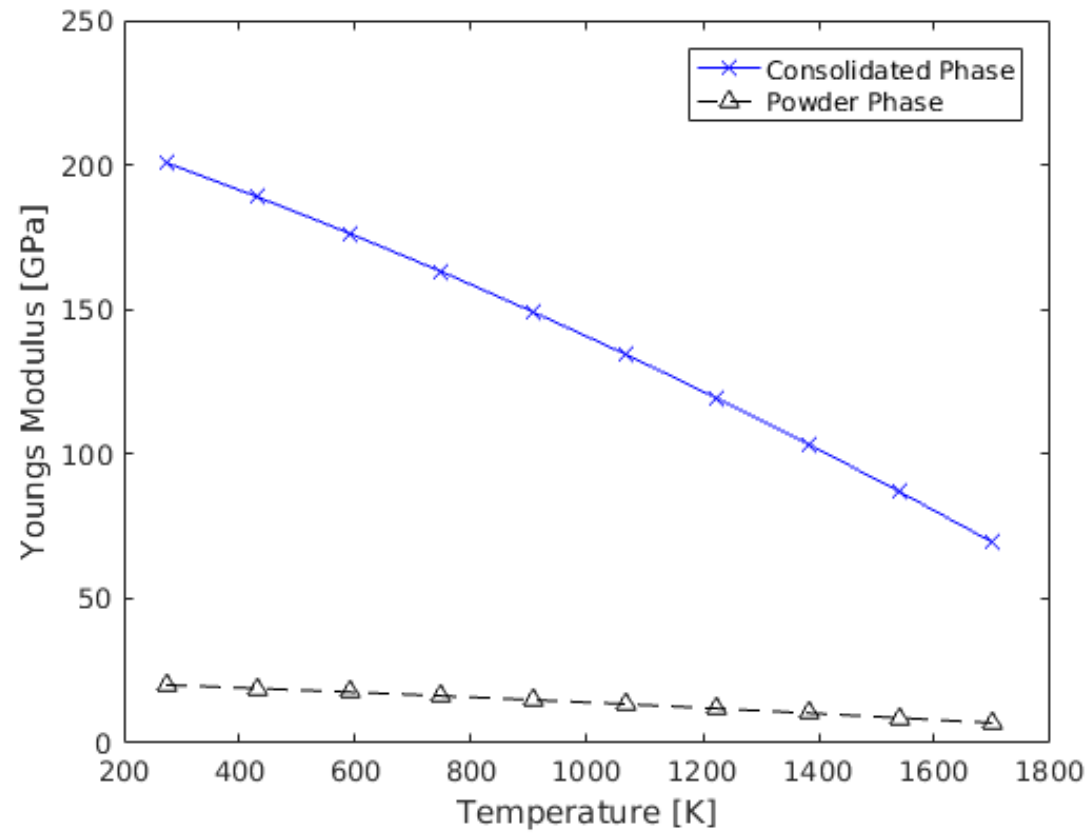

Figure 3.13 - Material 2 Temperature Dependent Young's Modulus [14]

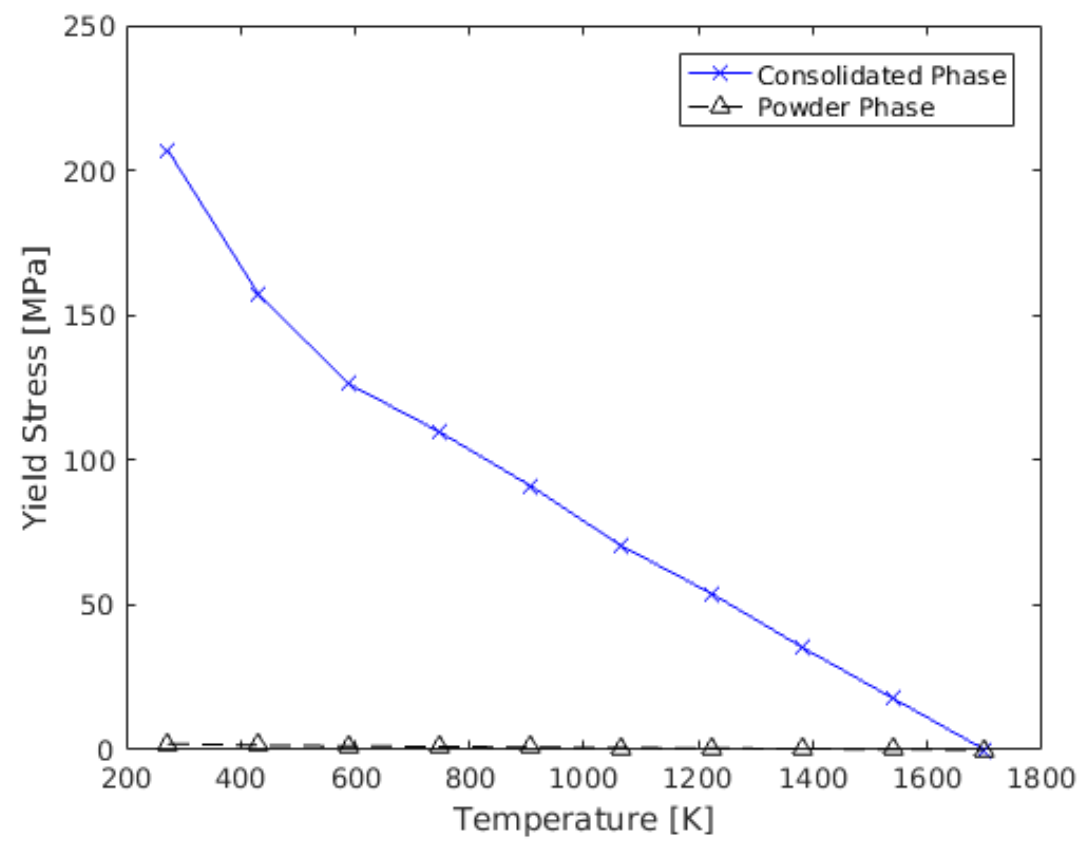

Figure 3.14 - Material 2 Temperature Dependent Yield Stress [14] 


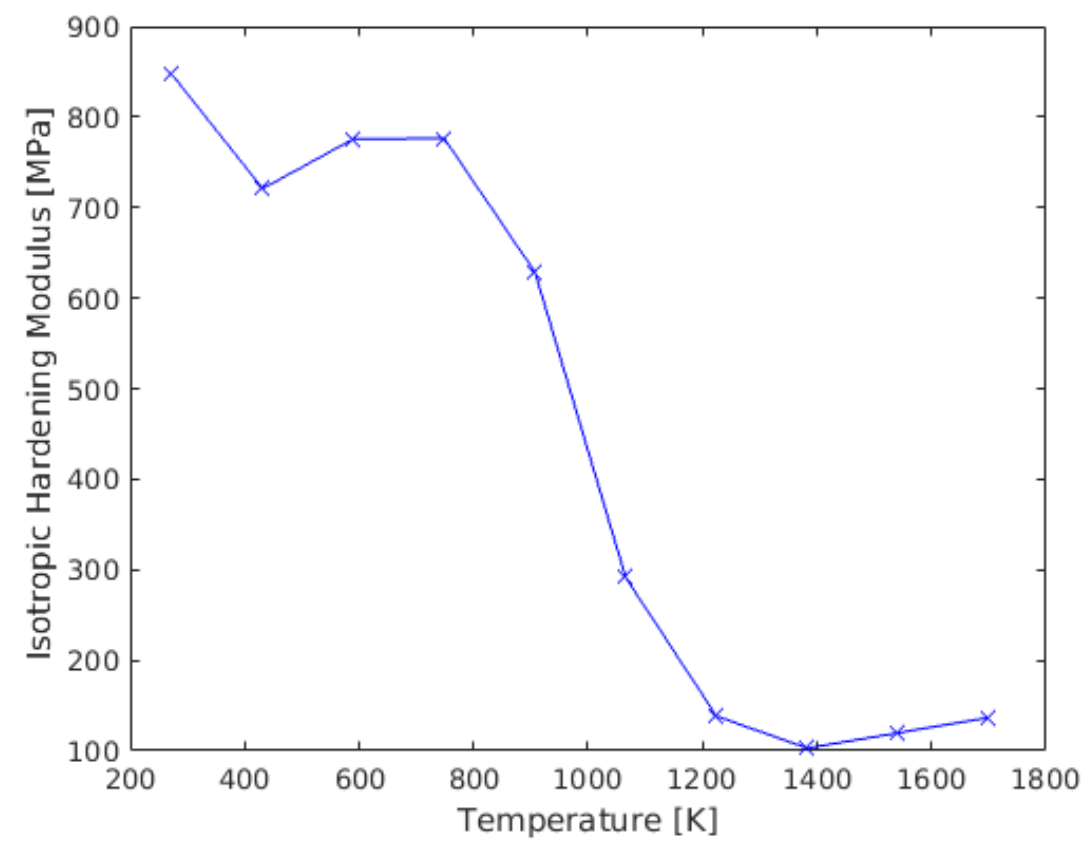

Figure 3.15 - Material 2 Temperature Dependent Isotropic Hardening Modulus [14]

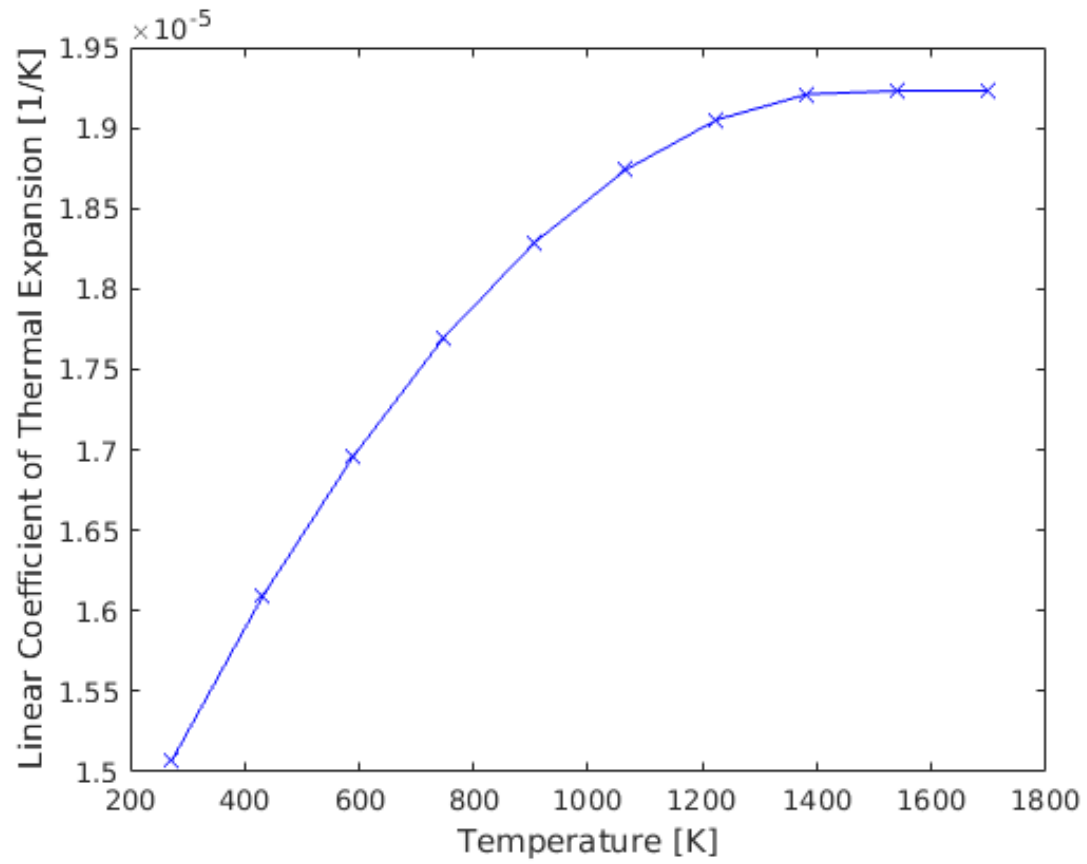

Figure 3.16 - Material 2 Temperature Dependent Coefficient of Thermal Expansion [14] 


\subsubsection{Material Model 3}

Material 3 is identical to material 1 except it used volume effective material properties for thermal conductivity, specific heat, Young's Modulus, and yield stress. In order to reduce the complexity of the model and the associated computational cost, the block support structure cross section shown in Figure 3.17 was replaced with the crosssection shown in 3.18 with volume effective material parameters. This approach is discussed in detail in $[62,64]$ and was also used in $[27,61]$.

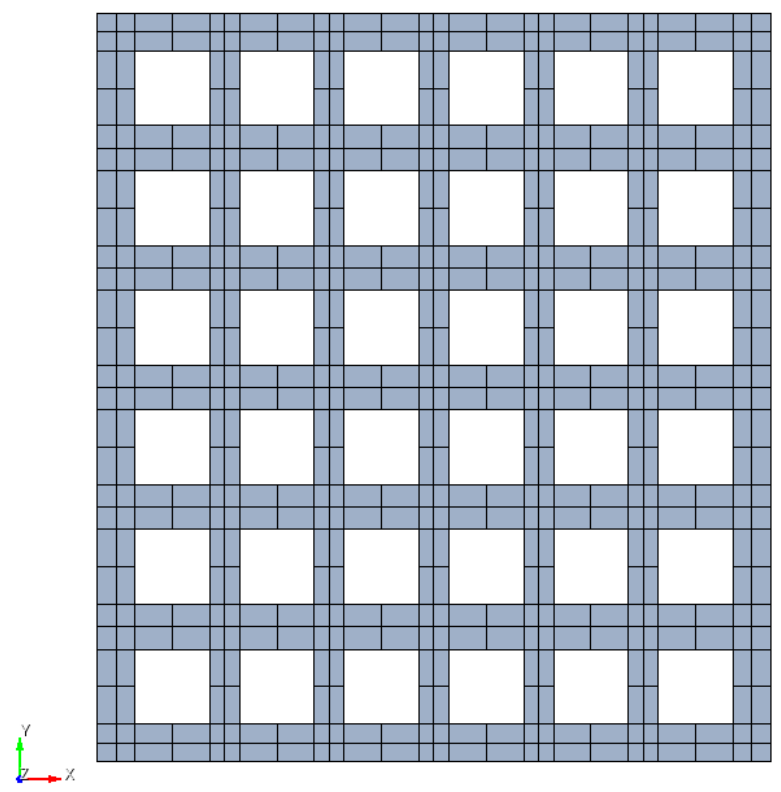

Figure 3.17 - Block Support Structure Meshed Cross Section 


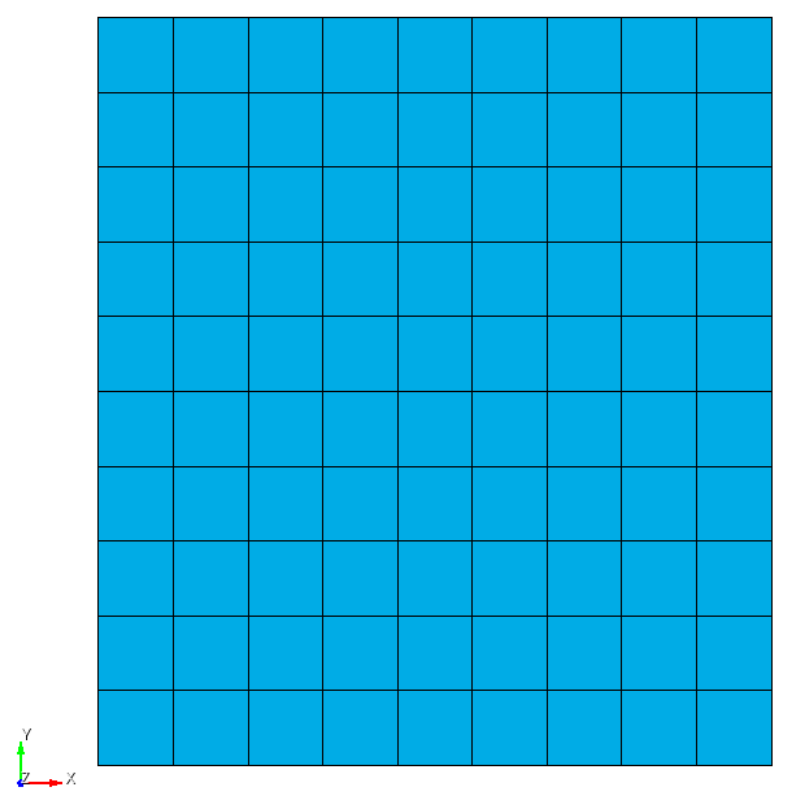

Figure 3.18 - Volume Effective Support Structure Meshed Cross Section

The equation for calculating the volume effective material properties is given by [62]:

$$
\Psi_{\text {effective }}=\phi \Psi_{\text {solid }}+(1-\phi) \Psi_{\text {powder }}
$$

where $\Psi$ is a material property and $\phi$ is the volume fraction of the consolidated support which is the same as the phase defined previously.

To validate the use of effective material properties, the two support structures shown above were subjected to the loading conditions shown in Figure 3.19. Figures 3.20 and 3.21 compare the temperature and displacement of the two support structures. There is no appreciable difference between the average temperatures, and the difference in displacement is relatively small compared to the overall magnitude of the displacement. 


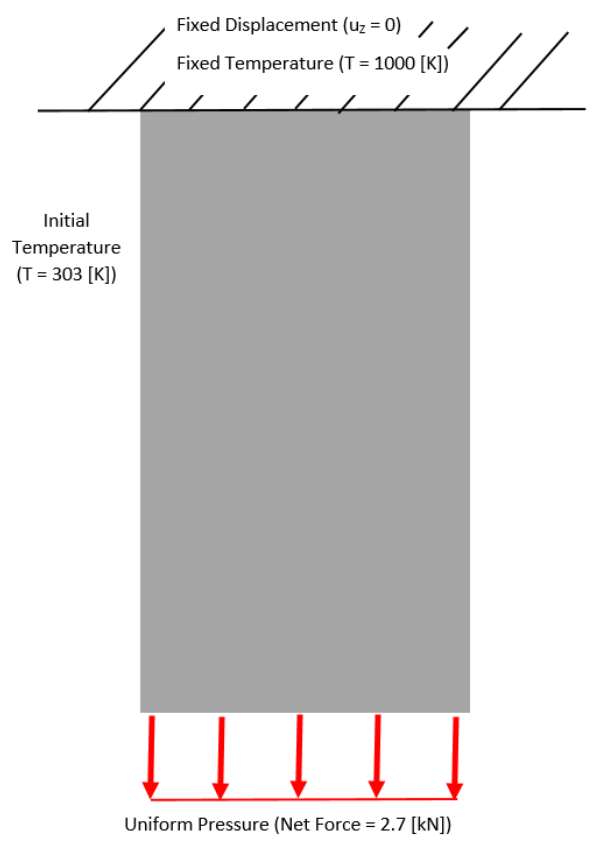

Figure 3.19 - Volume Effective Validation Loading Case

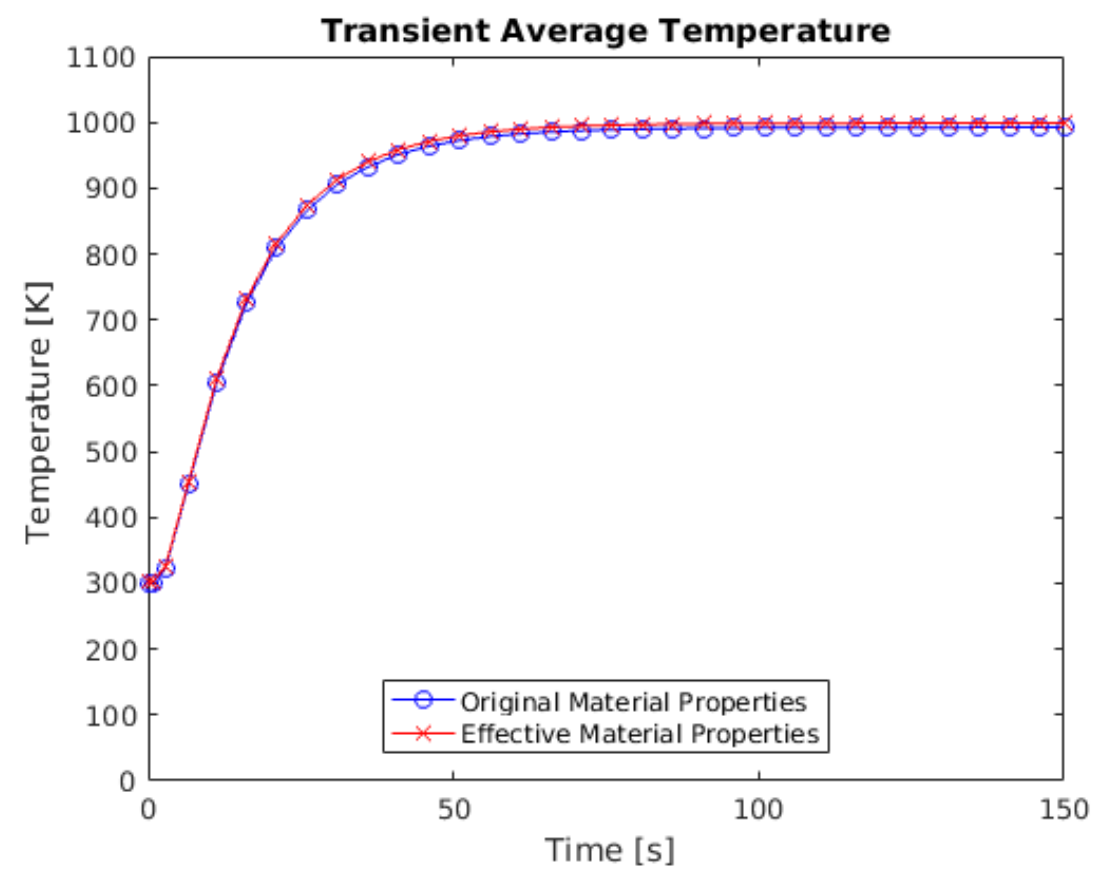

Figure 3.20 - Volume Effective Validation Average Bottom Surface Temperature 


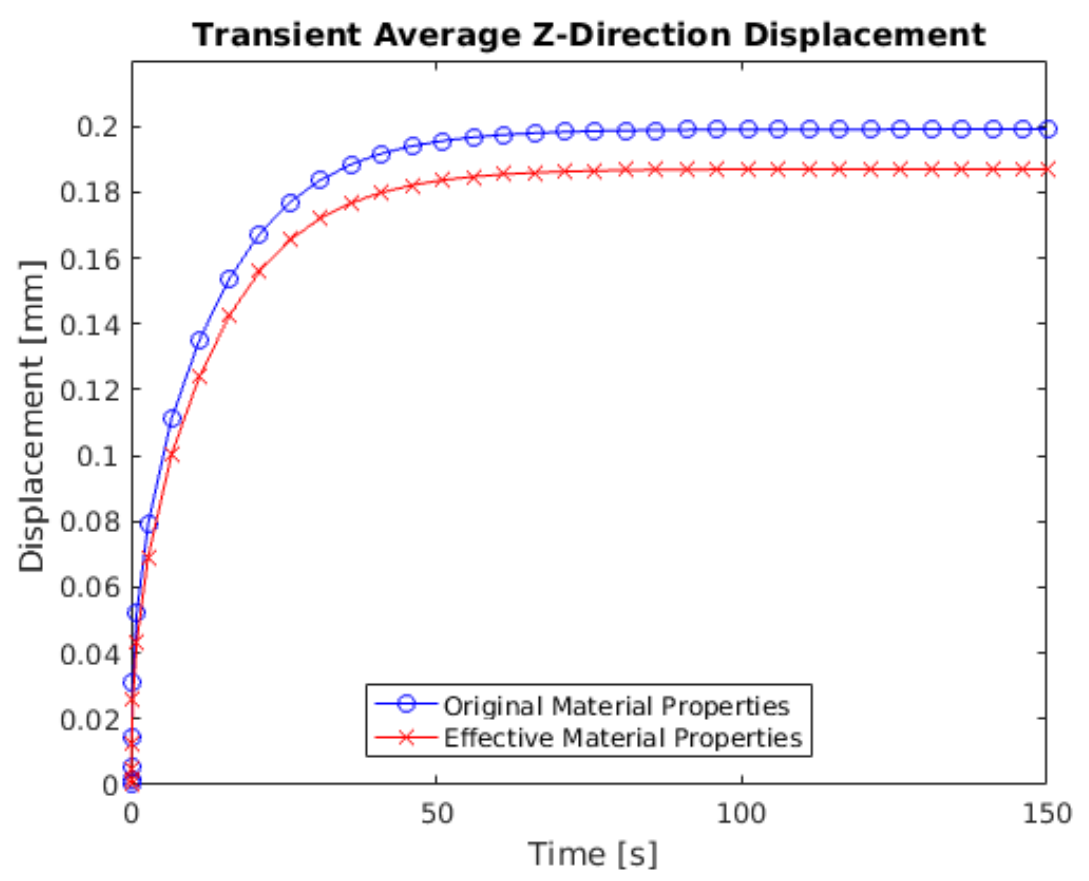

Figure 3.21 - Volume Effective Validation Average Bottom Surface Displacement

\subsubsection{Material Model 4}

The thermal conductivity of the powder trapped between the part and the support structure is much smaller than the consolidated thermal conductivity as seen in Figure 3.11, therefore it has little effect on the overall heat transfer. Due to the negligible effect of the powder's thermal conductivity the thermal model for material 4 is isotropic conduction where the thermal conductivity is set to $1 \%$ of the consolidate material's thermal conductivity and the heat capacity is the same.

Similarly, since the powder cannot sustain any tensile loads the stress material model is isotropic elastic, where the Young's modulus is set to $0.1 \%$ of the consolidate material's Young modulus and the Poisson's ratio is the same. 


\subsection{Numerical Implementation}

\subsubsection{Thermal Numerical Implementation}

The solution to the thermal model employs the weight residual formulation [16] which is discussed in great detail in $[34,65]$ and therefore will not be discussed here. Time integration for the thermal solution uses the generalized trapezoidal with $\alpha=1$, also known as backwards Euler method.

The phase of an element, $\phi$, can have two different meanings in the context of the SLM process. The first being the state of the matter: solid, liquid, or gas. The second ranges from the "powder phase" to the "consolidated phase" [16]. The "powder phase" represents unconsolidated solid powder while the "consolidated phase" represents both solid and liquid consolidated material. This definition of the phase is used to enforce unidirectional phase change from unconsolidated powder to consolidated substrate and the state phase change is addressed by varying material properties based on the temperature of the material [16]. For the purposes of this model the second description will be used where the subscripts $(\bullet)_{1}$ and $(\bullet)_{2}$ denote the powder phase and the consolidated phase, respectively.

The melt pool surrounding the laser heat source is modeled by tracking elements which contain the phase change interface, $\Gamma_{p}$. A detailed description of the phase change numerical implementation and validation is available in [16].

Similar to other part scale models $[17,31,61]$, the present work incorporates a global model of the SLM process where the model powder layer depth $(L)$ was considerably larger than the physical powder layers. To account for the increase in layer thickness and to further reduce the computational cost the speed $(v)$, power $(p)$, and radius $(R)$ of the laser heat source also differed from typical machine parameters. 
All of the models in this work used the following setting:

$$
\begin{aligned}
L & =0.5[\mathrm{~mm}] \\
v & =23[\mathrm{~mm} / \mathrm{s}] \\
R_{1} & =1[\mathrm{~mm}] \\
R_{2} & =0.35[\mathrm{~mm}]
\end{aligned}
$$

The powder layer depth is an order of magnitude larger than typical physical layer depths, the model velocity is an order of magnitude less the typical laser velocities, and the two radii are an order of magnitude larger than the typical maximum and minimum laser radii.

The laser heat source was implemented in the simulations using two mesh independent body loads (MIBL). In either MIBL, a cylindrical volume defined by $L$ and $R_{1}$ or $R_{2}$ with a uniform volumetic heat input followed a laser path, similar to the one shown in Figure 3.22.

The first MIBL, which used the larger radius $\left(R_{1}\right)$, was applied to the entire model, except for the contact area teeth because $R_{1}$ is considerably larger than the Top Lengths. The second MIBL, with radius $\left(R_{2}\right)$, modeled the heat source which melted the powder to form the contact area teeth. For each layer, the first MIBL traced the perimeter of the layer and then completed parallel passes which overlap by one radius. The second MIBL completed single passes centered over each tooth, and then repeated all the passes. After each layer, the part was allowed to cool for a downtime of $70[\mathrm{sec}]$ based on a downtime measured in the experimental validation build. Both MIBLs used laser powers that were tuned to ensure that the powder melted fully while keeping the maximum temperature below $9000[\mathrm{~K}]$. 


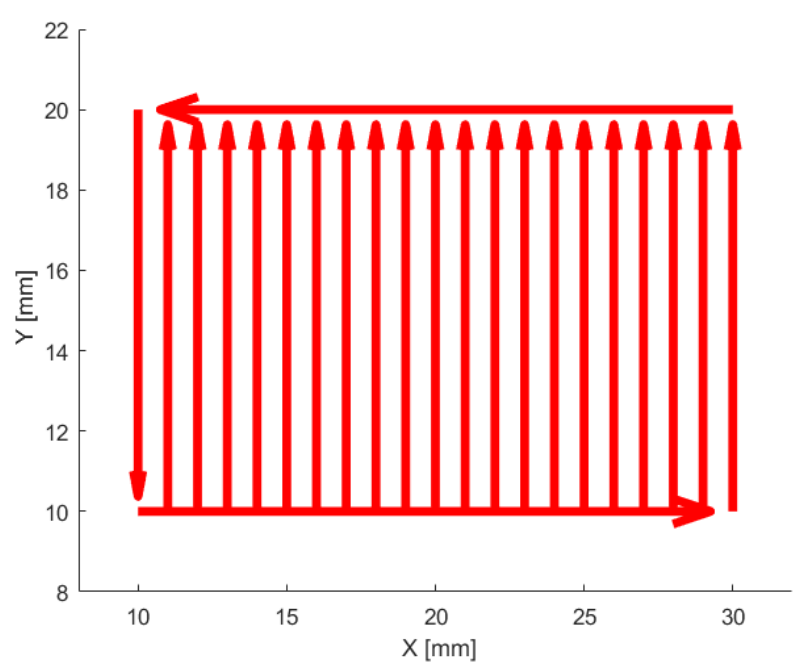

Figure 3.22 - Global Model Laser Path

\subsubsection{Thermal Initial/Boundary Conditions}

\subsubsection{Initial condition}

In order to reduce the computational cost, the first $12[\mathrm{~mm}]$ of the part and support structure were modeled as consolidated substrate. To account for the heat accumulation in the first $12[\mathrm{~mm}]$ of the part and support structure the nonuniform temperature profile shown in Figure 3.23 was assigned as an initial condition. The temperature profile was generated using the average layer temperatures from a simulation of the first $12[\mathrm{~mm}]$ of the part using $L=1[\mathrm{~mm}]$. The initial temperature profile corresponds to the time just prior to the addition of the next layer in the full part model. 


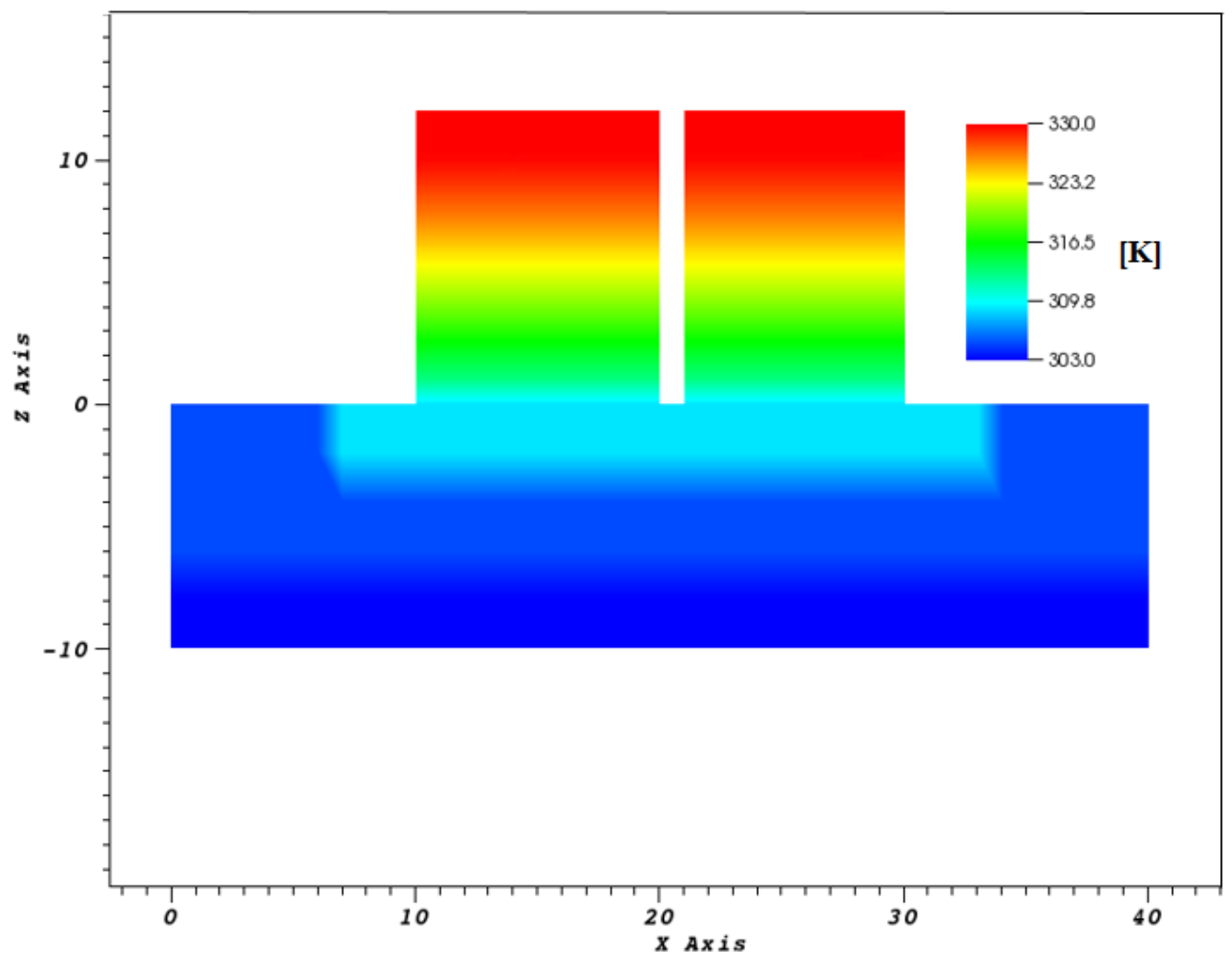

Figure 3.23 - Initial Temperature Distribution

\subsubsection{Boundary conditions}

A dirchlet boundary condition, a constant temperature $T=303[K]$, was assigned at the boundary, $\Gamma_{T}$, which is the bottom surface of the base plate. The effects of radiation and evaporative cooling along the top of the solidifying layer can be modeled in Diablo [14]; however, this increases the computational cost considerably. The models in this study assumed all surfaces, except $\Gamma_{T}$ were adiabatic. The effects of radiation and evaporative cooling are accounted for by the tuned laser powers. 


\subsubsection{Thermo-Mechanical Numerical Implementation}

A detailed discussion on the solution to the Equations 3.11 - 3.16 is available in [66]. Time stepping in the solid mechanics model utilizes the Newmark method and the staggered coupling between the thermal and solid mechanics is shown in Figure 3.24 $[16]$.

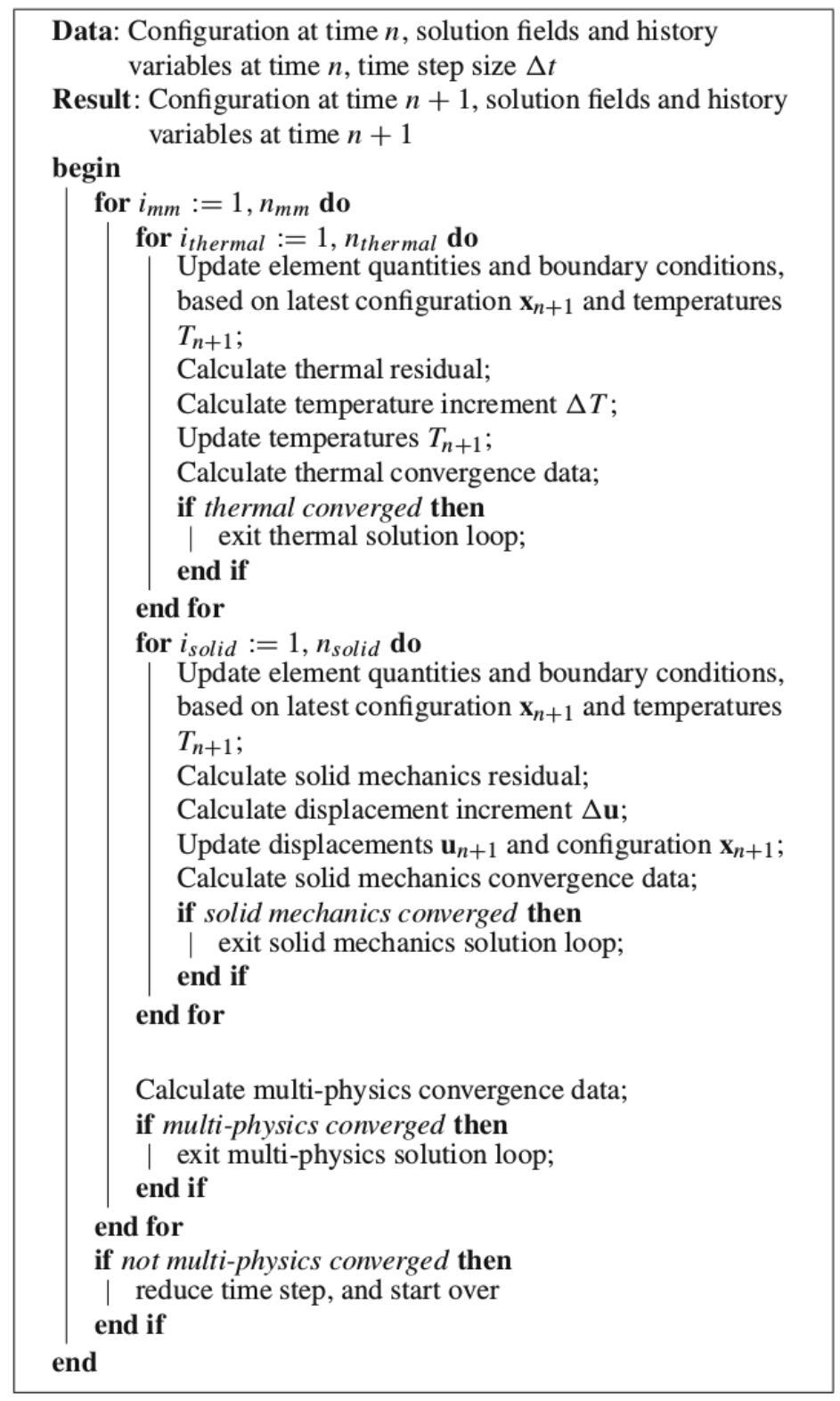

Figure 3.24 - Thermo-Mechanical Coupling Algorithm [16] 


\subsubsection{Solid Mechanics Initial/Boundary Conditions}

\subsubsection{Initial condition}

The first $12[\mathrm{~mm}]$ of the part and support modeled as consolidated substrate would have accumulated residual stresses during the SLM process; however, the stress in the lower part and support were not of interest in this study so the entire model was initially stress free, traction free, and had no deformation.

\subsubsection{Boundary conditions}

The portion of the boundary corresponding to displacement boundary conditions, $\Gamma_{u}$, is the bottom and sides of the base plate. The displacement in the direction normal to each surface was fixed. This allows for the base plate to expand and contract with the thermal load, but restricted the base plate from moving.

\subsection{Domain Discretization}

The meshed domain of Test 4 is shown in Figure 3.25. The domain was discretized to ensure high quality elements, as measured by the scaled jacobian. To obtain elements with minimal distortion from the ideal hex element shape, surfaces parallel to the $\mathrm{X}$ Y plane were meshed. These meshes were then swept through the Z-direction. The nodes in the Z-direction were spaced $2[\mathrm{~mm}]$ apart in the base plate, $1[\mathrm{~mm}]$ apart in the lower part, and $0.5[\mathrm{~mm}]$ apart in the contact area and overhanging feature. The spacing of nodes in the X-direction can be seen in Figure 3.1 and the nodes in the Y-direction were spaced $1[\mathrm{~mm}]$ apart as shown in Figure 3.27.

The meshes for Tests 1-3 are very similar to the mesh of Test 4, therefore these meshes are not pictured. 


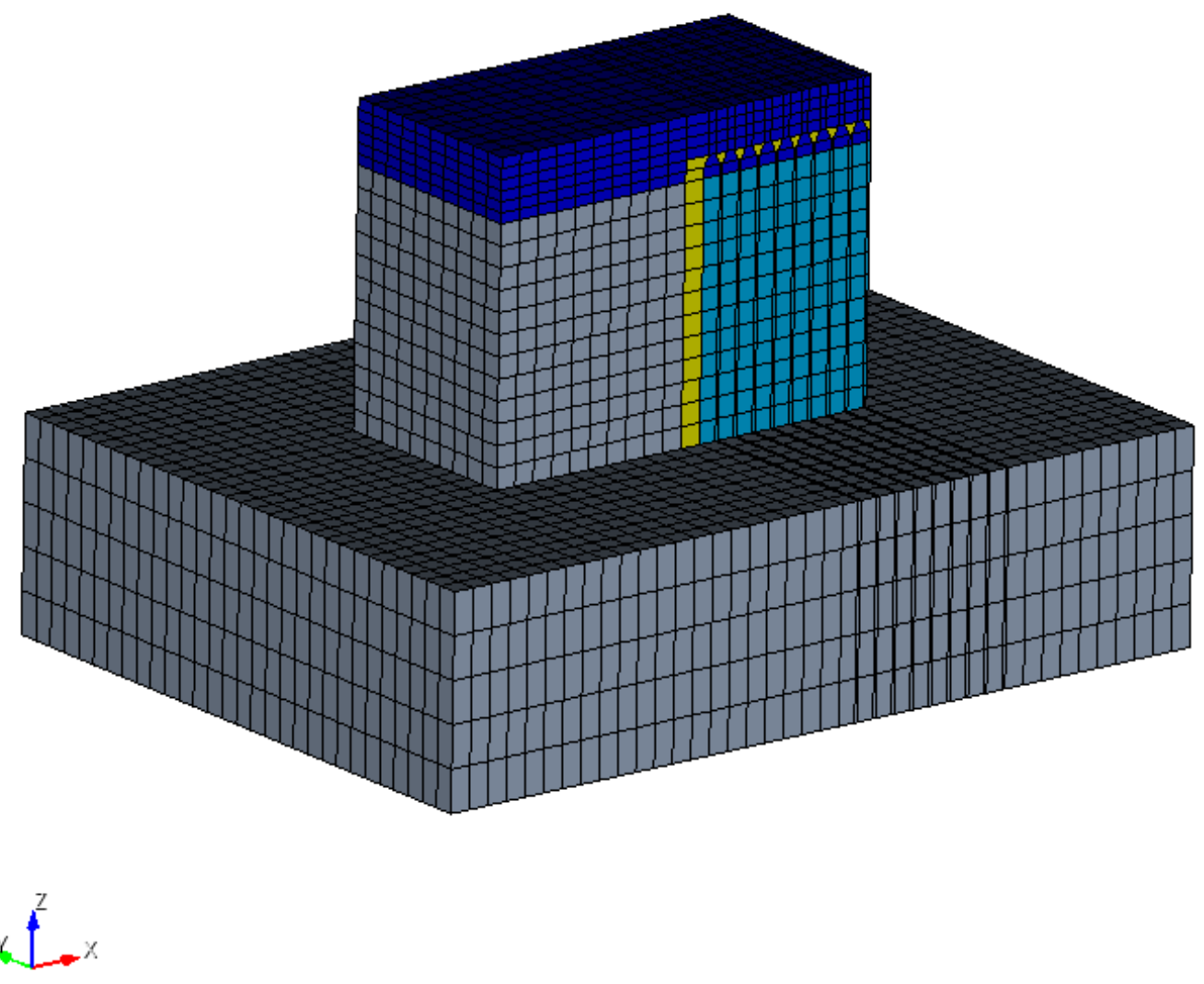

Figure 3.25 - Isometric View of Test 4 Mesh 


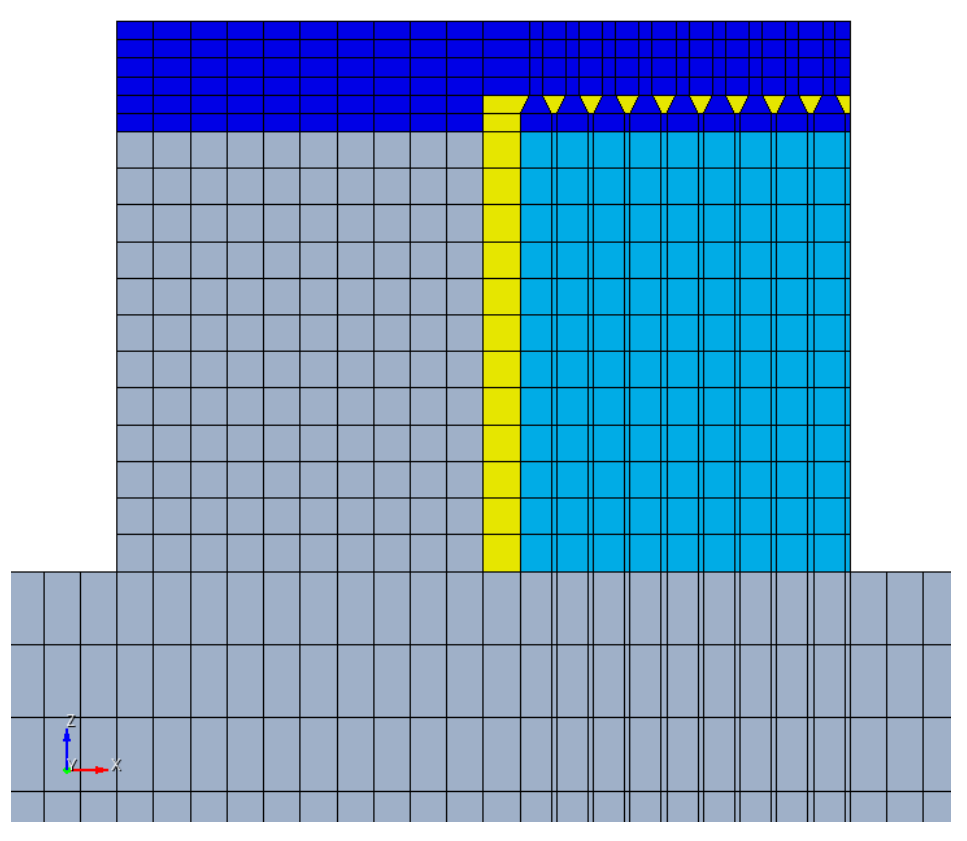

Figure 3.26 - Side View of Test 4 Mesh

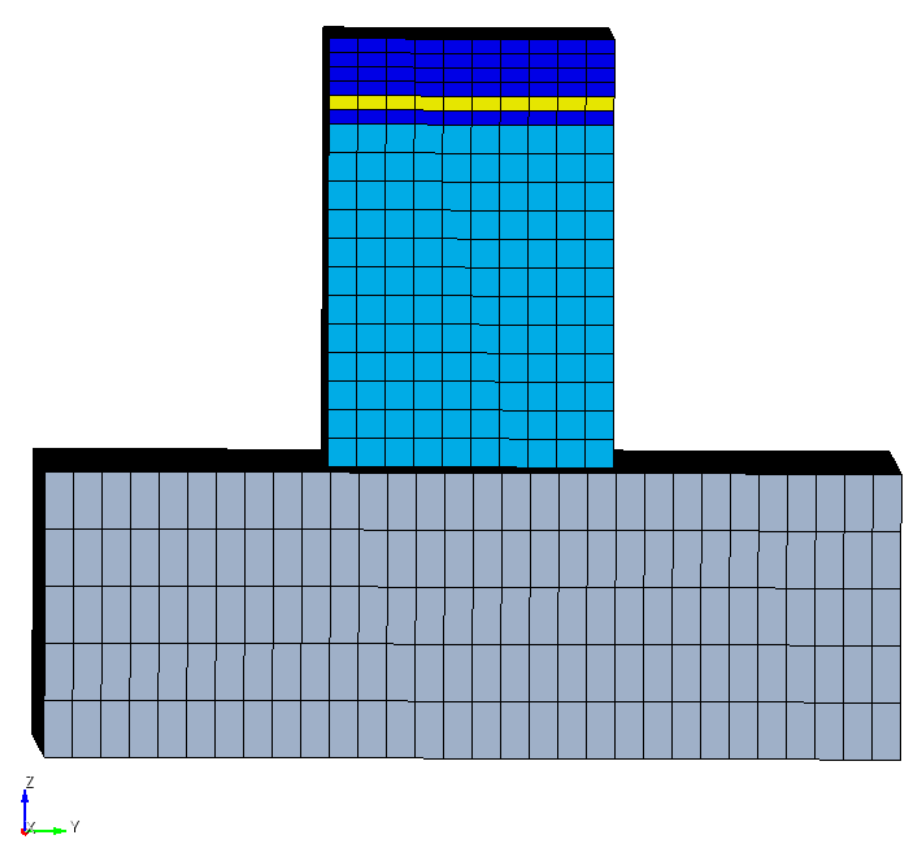

Figure 3.27 - Front View of Test 4 Mesh 


\subsection{Mesh Dependence}

Prior to modeling the SLM process for any of the four test cases, the mesh dependence of the solution was determined. Figures 3.28 and 3.29 show the mesh dependence of the thermal solution in the part and the support structure, respectively. The model used for this mesh dependence study simulated the fabrication of a single layer of the part and support structure. The part consisted of hex elements with side lengths of $0.5[\mathrm{~mm}], 1[\mathrm{~mm}]$, and $2[\mathrm{~mm}]$ which correspond to $h_{1}, h_{2}$, and $h_{3}$ respectively. The cross-section shown in Figure 3.17 was meshed based on line segment intervals, and then swept in the Z-direction with $1[\mathrm{~mm}]$ hex elements. The mesh shown in Figure 3.17 corresponds to an interval of 2 elements per line segment. In Figure $3.29 h_{1}, h_{2}$, and $h_{3}$ correspond to intervals of 4,2 , and 1 element(s) per line segment, respectively.

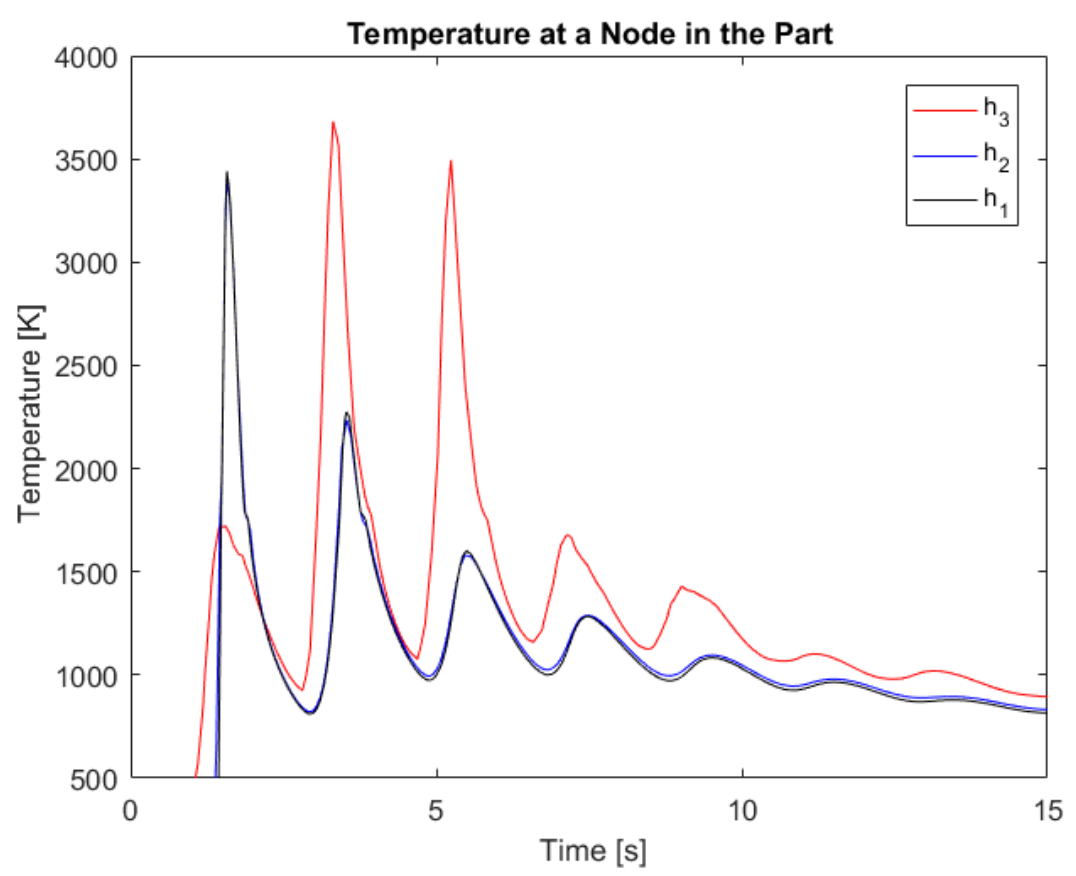

Figure 3.28 - Thermal Mesh Dependence in the Part 


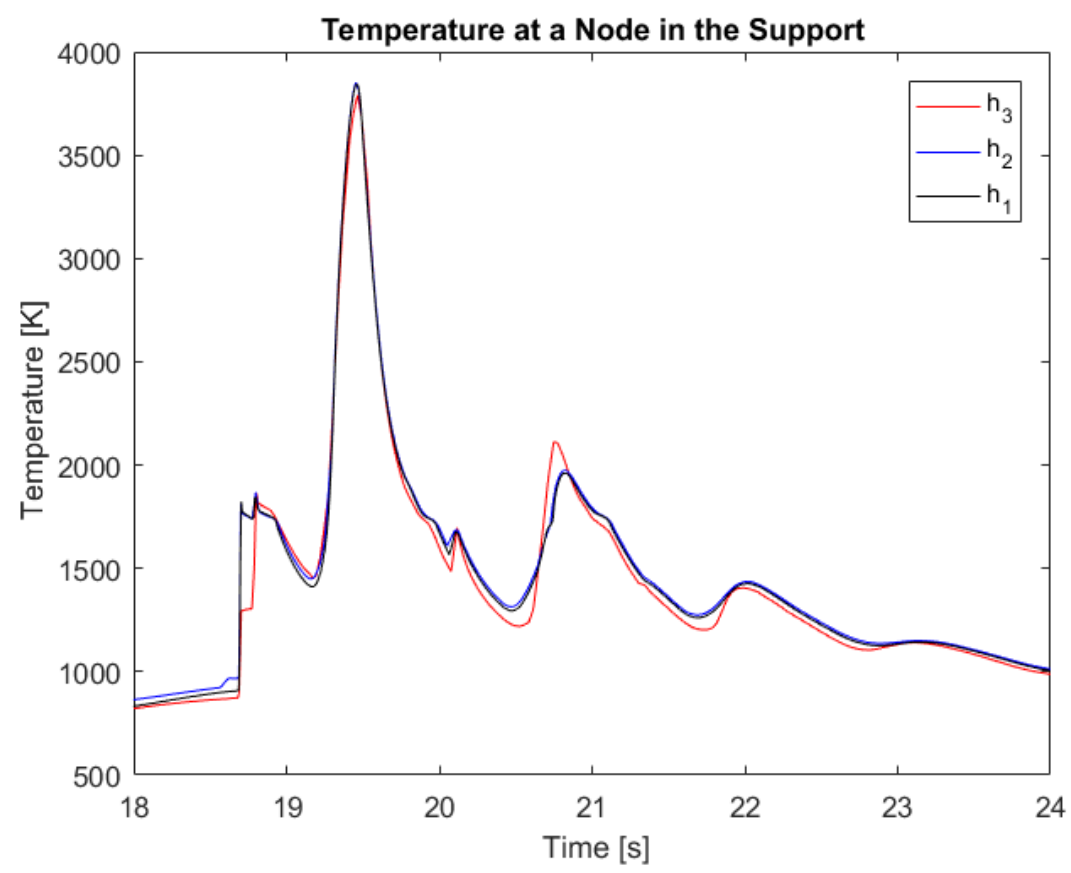

Figure 3.29 - Thermal Mesh Dependence in the Support

Figure 3.30 shows the average percent change of the nodal temperatures in both the part and the support as a function of time. The values of $\Delta T^{32}$ and $\Delta T^{21}$ were calculated with Equations 3.28 and 3.28. Using the percent change from one mesh size to another as a quantitative measure of the mesh dependence, the meshes corresponding to $h_{2}$ are considered to be converged since the average value of $\Delta T^{21}$ is $1.36 \%$.

$$
\begin{aligned}
\Delta T^{32}(t) & =\frac{1}{N} \sum_{n=1}^{N} 100 \% \frac{T_{n}^{3}(t)-T_{n}^{2}(t)}{T_{n}^{3}(t)} \\
\Delta T^{21}(t) & =\frac{1}{N} \sum_{n=1}^{N} 100 \% \frac{T_{n}^{2}(t)-T_{n}^{1}(t)}{T_{n}^{2}(t)}
\end{aligned}
$$




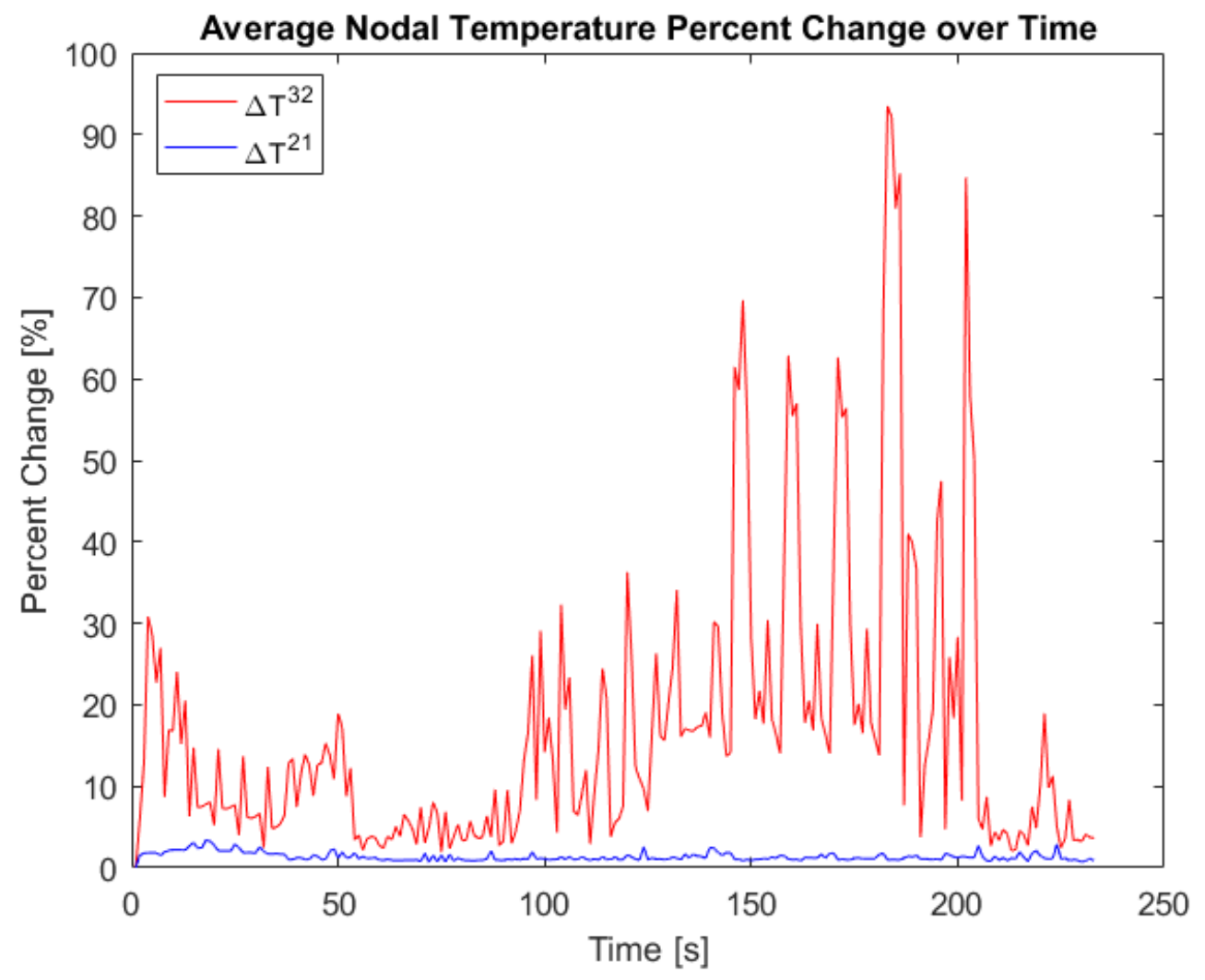

Figure 3.30 - Quantitative Measure of Thermal Mesh Dependence

Figures 3.31-3.33 show the mesh dependence of the stress in the $\mathrm{X}, \mathrm{Y}$, and $\mathrm{Z}$ directions under a uniaxial tensile load. Each mesh consisted of hex elements with side length ranging from $2[\mathrm{~mm}]$ to $0.25[\mathrm{~mm}]$. 


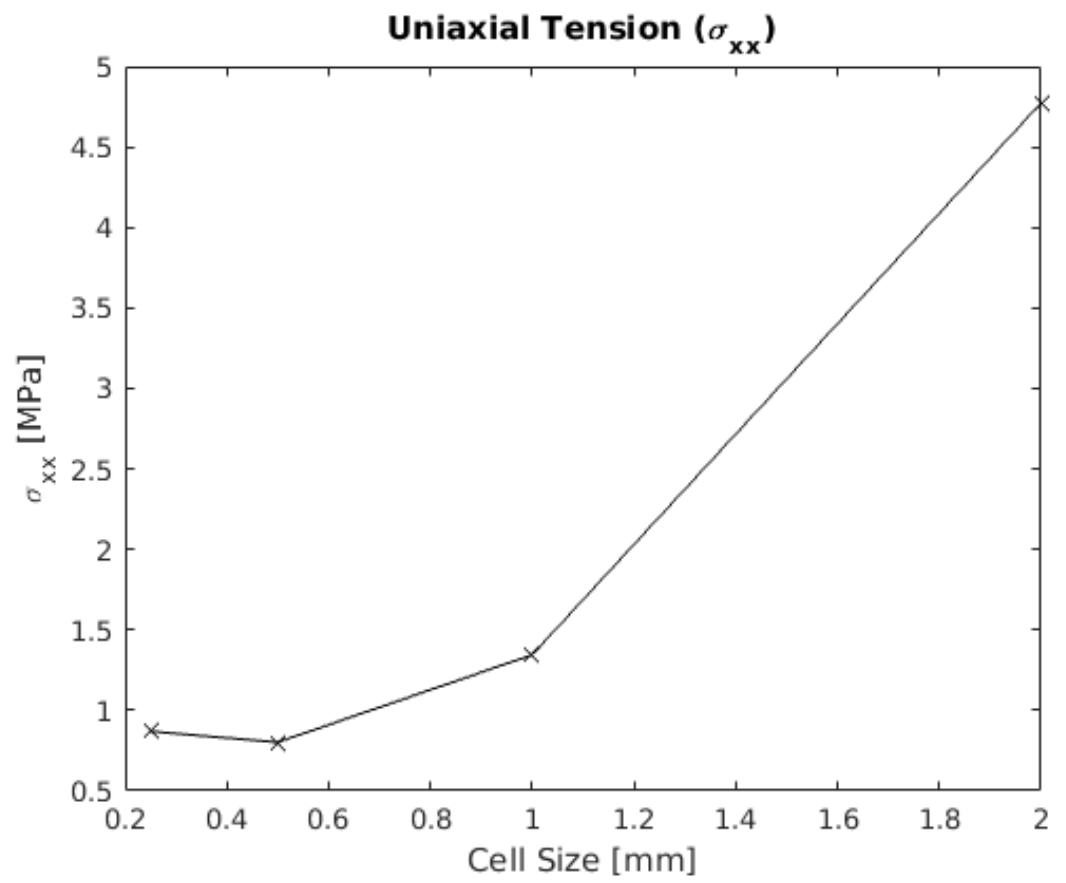

Figure 3.31 $-\sigma_{x x}$ Mesh Dependence Under Tensile Load

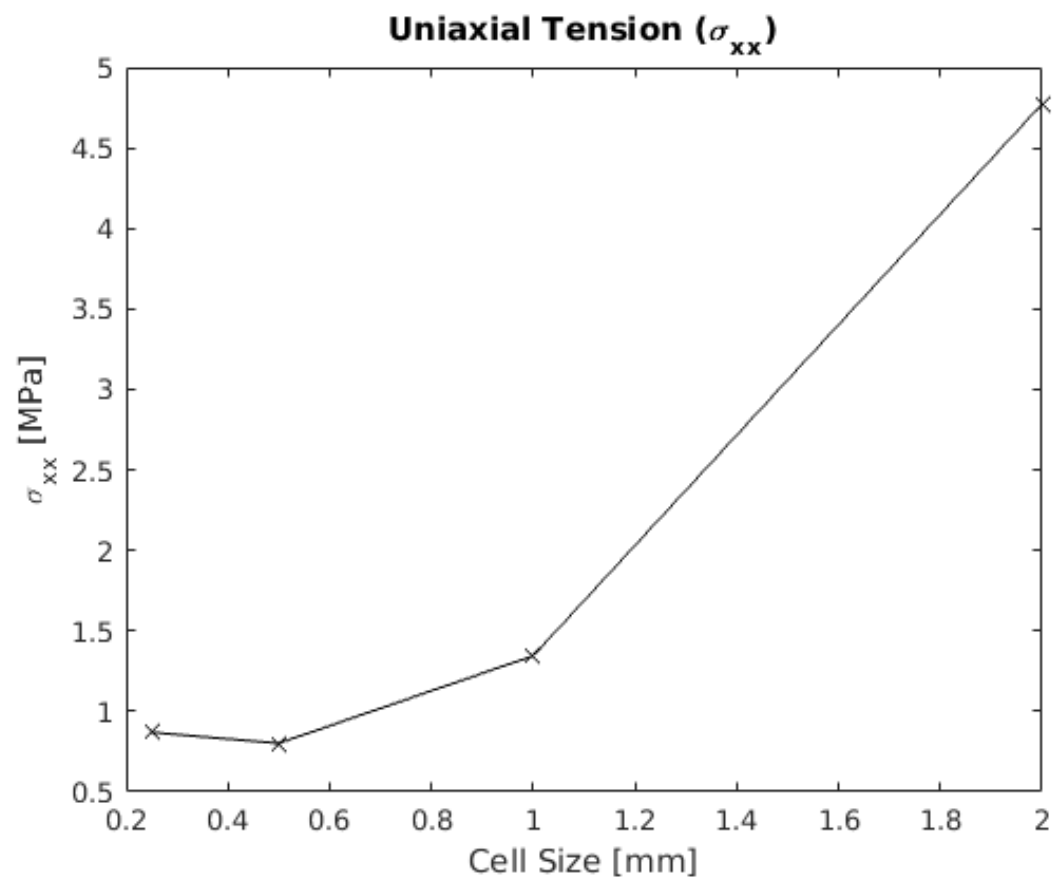

Figure 3.32 $-\sigma_{y y}$ Mesh Dependence Under Tensile Load 


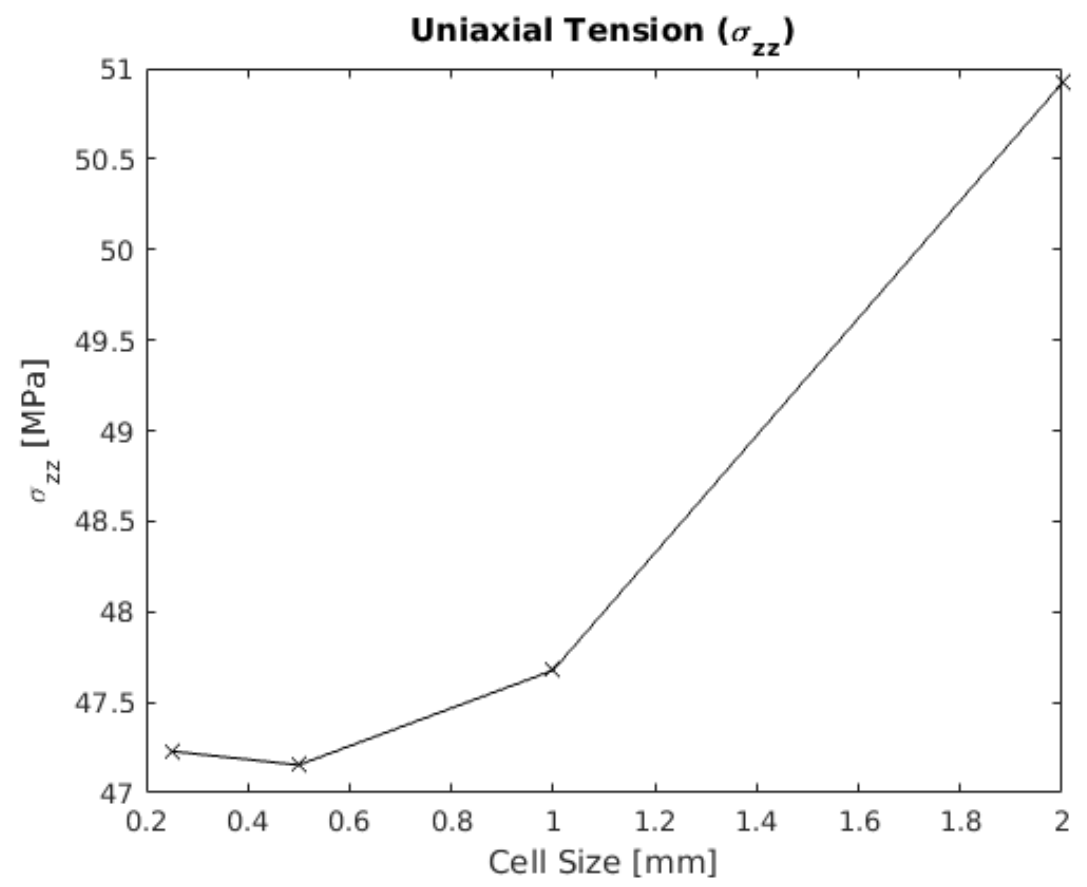

Figure 3.33 $-\sigma_{z z}$ Mesh Dependence Under Tensile Load

Figure 3.34 shows the residual stress in the Z-direction in the bottom of the part after seven layers have been consolidated on the base plate. In this model the hex element cell sizes ranged from $2[\mathrm{~mm}]$ to $0.5[\mathrm{~mm}]$. From visual inspection, the thermo-mechanical solution was assumed to be converged at an average cell size of 1 $[\mathrm{mm}]$. 


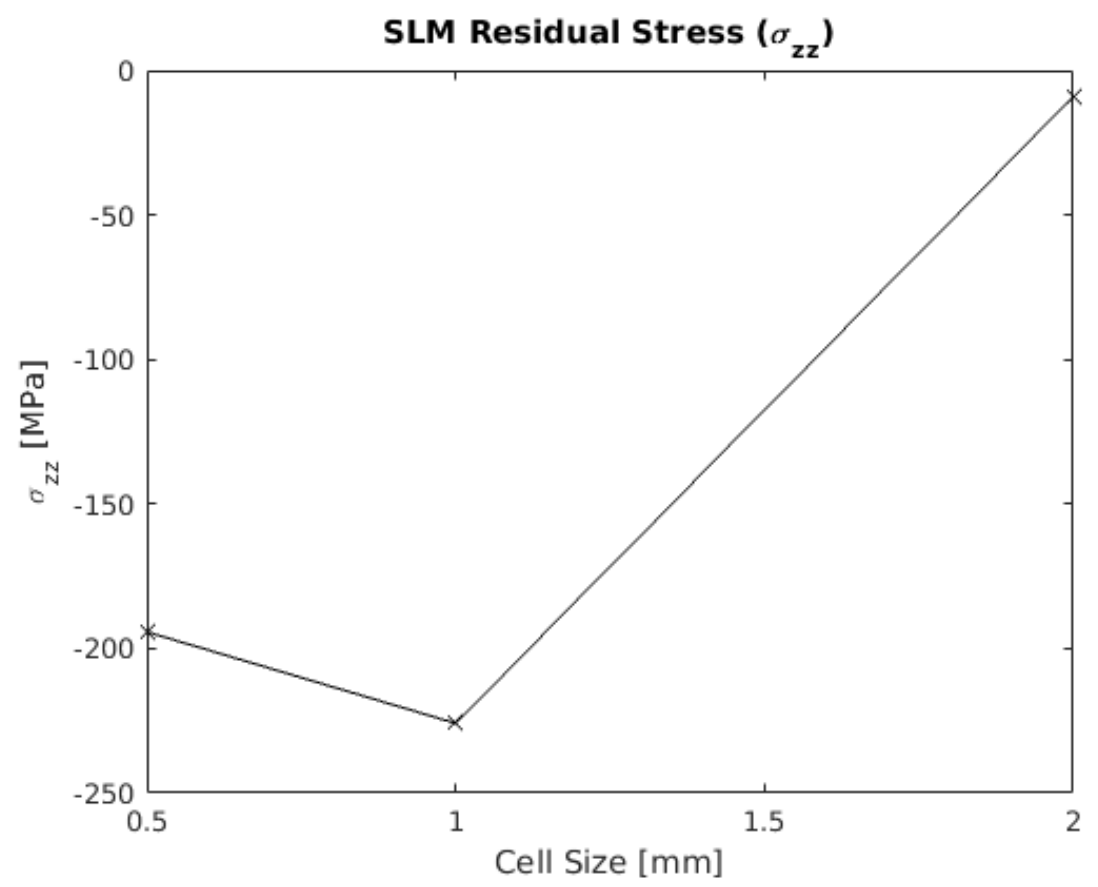

Figure 3.34 - Residual Stress Mesh Dependence

\subsection{Experimental Validation}

Figure 3.35 is a top view of parts on a SLM-125 build plate. Fourteen overhang parts with seven different support structures were built using an average velocity of 600 $[\mathrm{mm} / \mathrm{s}]$ and an average power of $200[\mathrm{~W}]$. Two of the parts in the build used the support from Test 1 and two of the parts used the support from Test 2, the specific support parameters for Tests 1-4 can be seen in Table 3.1. The height of each part, in the Z-direction, was measured with a Brown Sharpe PFX coordinate measuring machine (CMM) before and after the support structure was detached from the base plate. Figure 3.36 is a picture of a part after the support has been separate from the base plate using wire electrical discharge machining (EDM). 


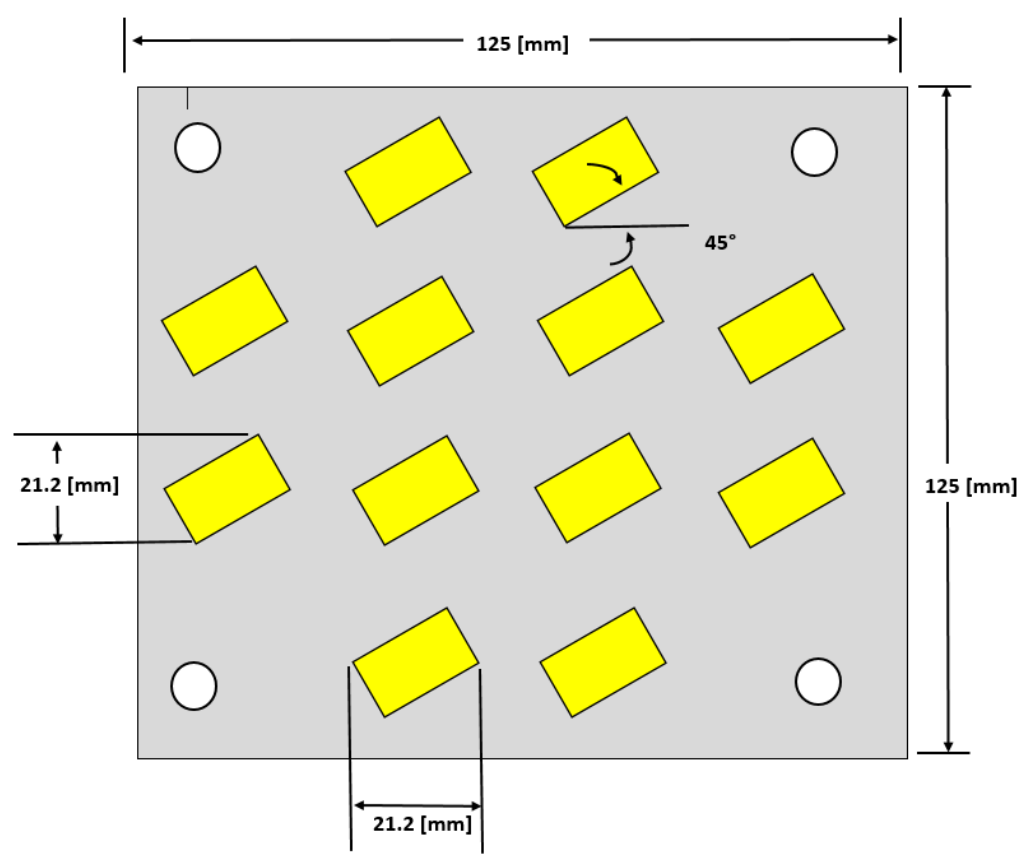

Figure 3.35 - Experimental Build Plate Layout

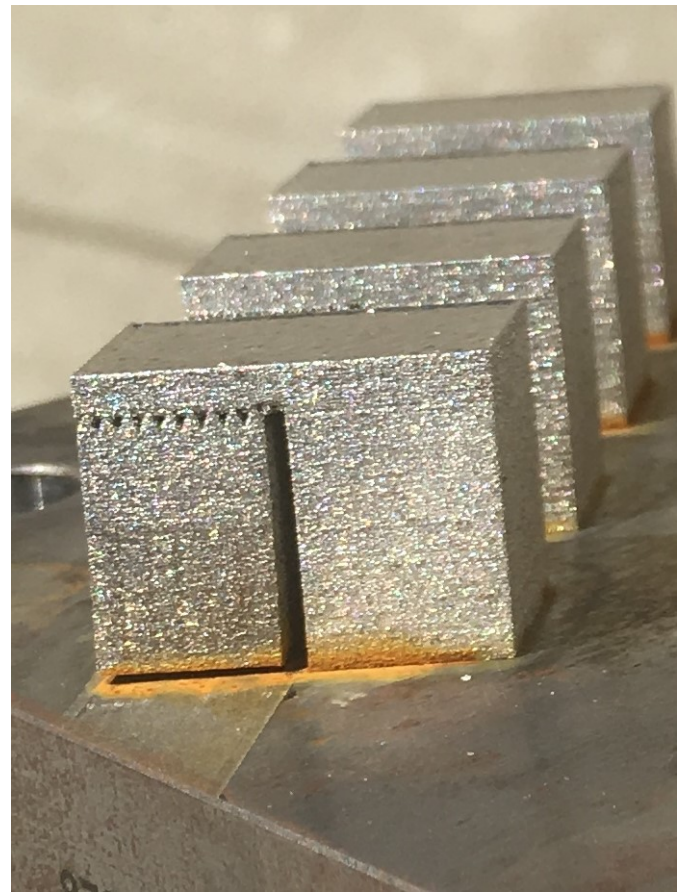

Figure 3.36 - Detached Support 
Approximately 100 sample points were taken along the top of each surface. The deflection of the surface, after the detachment of the support structure, was compared to the deflection of the overhang in the model after the support structure elements were deactivated. A comparison between the experimental and model deflection at a particular Y-value can be seen in Figures 3.37 and 3.38. Comparisons between the experimental and model deflections at other Y-values can be seen in Appendix A. While the model over-predicted the deflection at the end of the overhang for Test 1, the model does capture the trend and the relative magnitude of the deflections. Considering the complexity of the SLM process, the experimental data and the model results show relatively good agreement. The model deflections were calculated from the nodal displacements on the down-skin side of the overhang, therefore negative deflection values between $20<X<24$ [mm] were removed from the data set. The negative deflection at these locations was caused by large unsupported sections on the down-skin surface and do not reflect the deflections at the top surface.

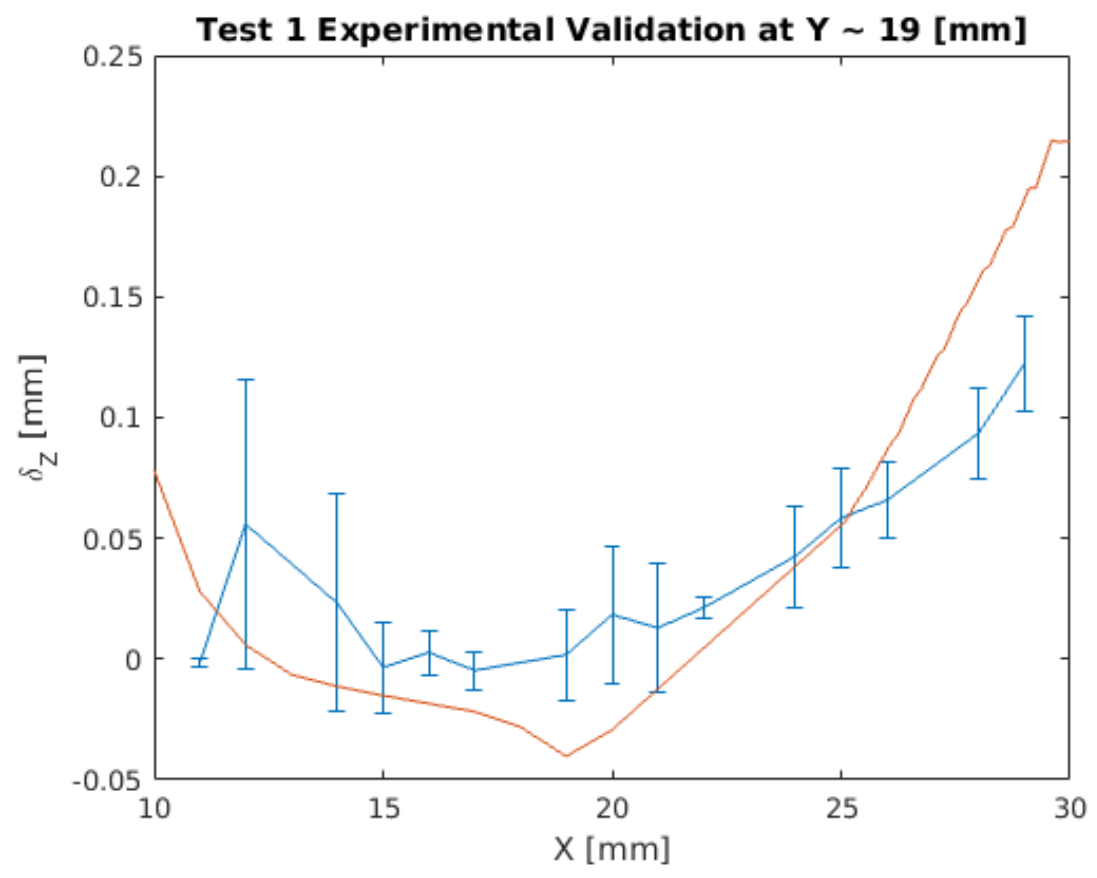

Figure 3.37 - Test 1 Experimental Validation at $\mathrm{Y} \approx 19[\mathrm{~mm}]$ 
Test 2 Experimental Validation at $Y \sim 19[\mathrm{~mm}]$

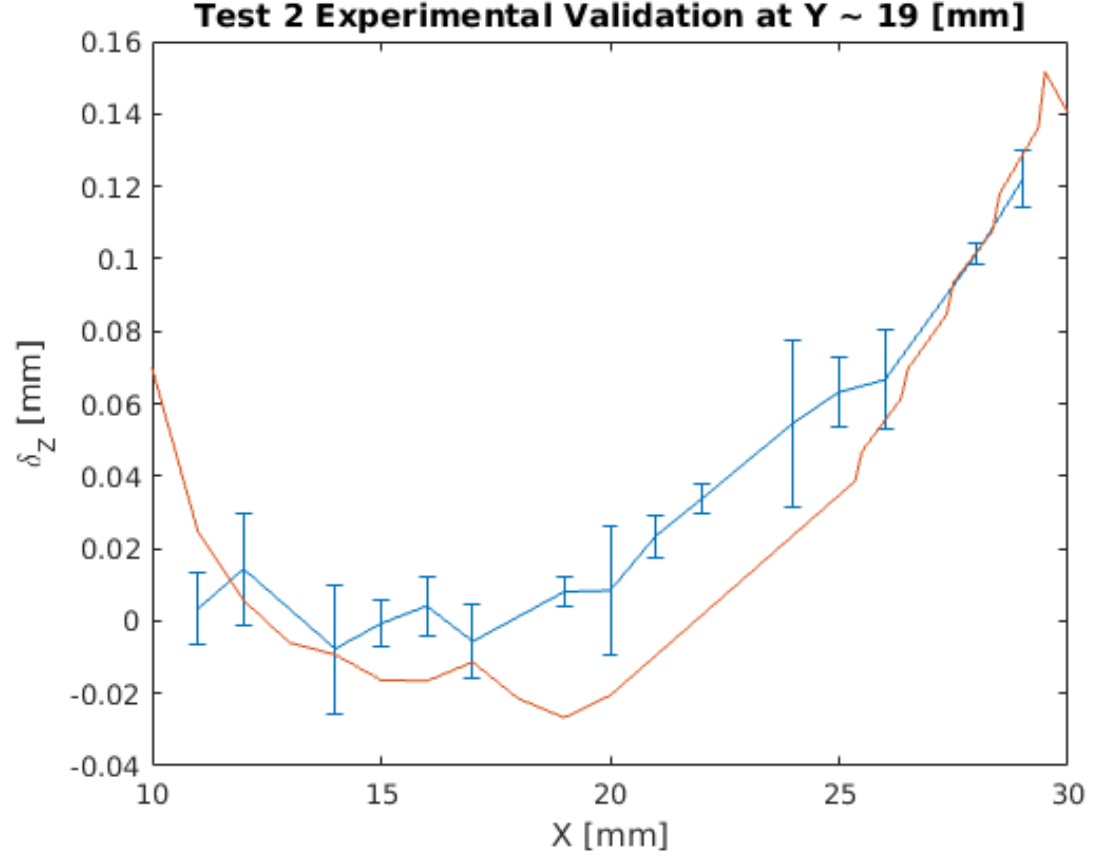

Figure 3.38 - Test 2 Experimental Validation at $\mathrm{Y} \approx 19[\mathrm{~mm}]$ 


\section{CHAPTER 4}

\section{Results \& Discussion}

This section presents the simulation results for the four support structure configurations describe in Table 3.1 and a discussion on the significance of the results. This chapter consists of three sections; in-plane residual stresses, axial residual stresses, and the effect of the contact area parameters on the residual stresses. Figure 4.1 provides the dimensions for the overhang region plots which are shown throughout the chapter. For reference, the unsupported overhang region will be used to describe the region $20[\mathrm{~mm}] \leq X \leq 21[\mathrm{~mm}]$.

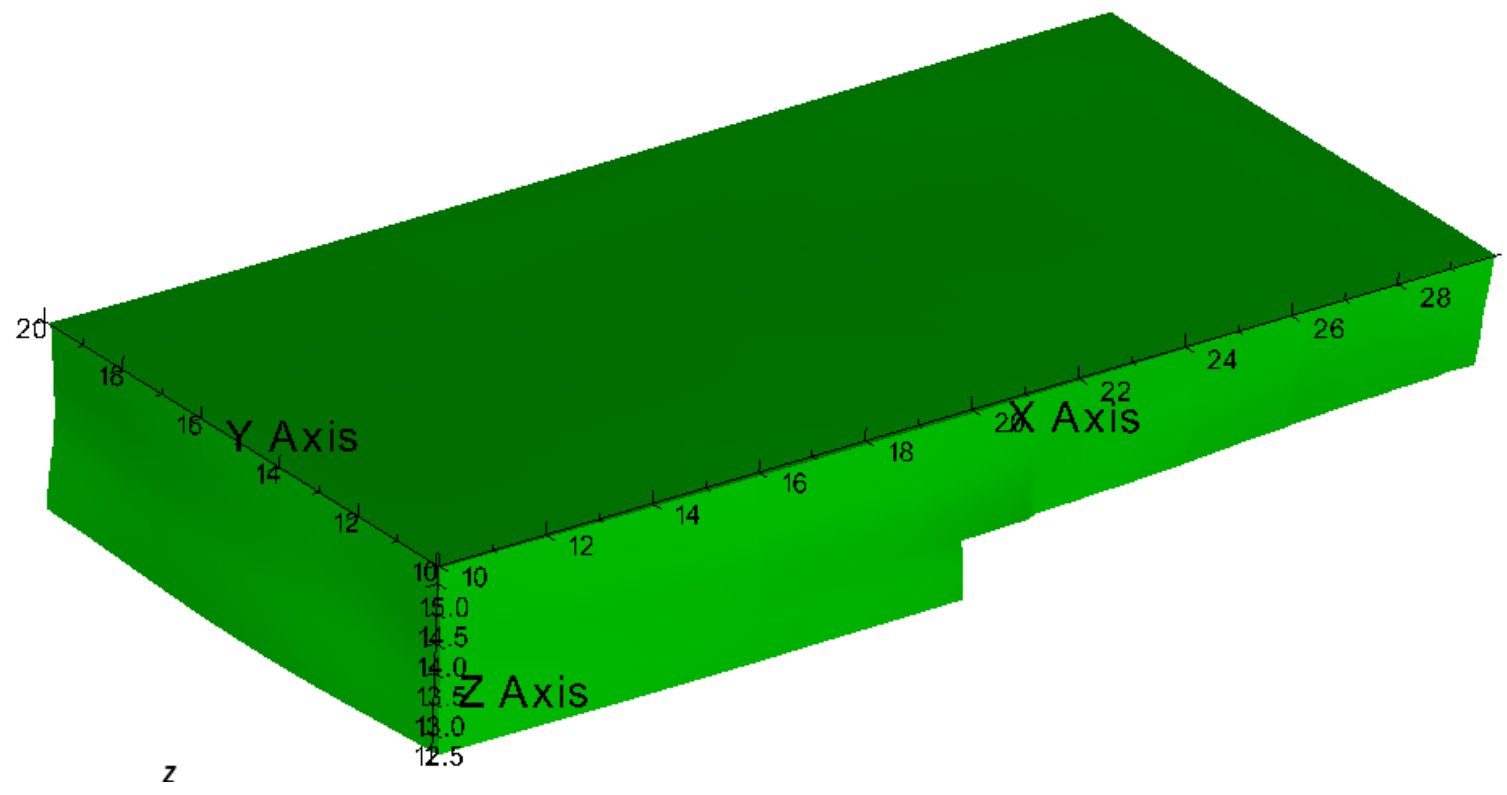

Figure 4.1 - Overhang Section Dimensions 


\subsection{In-Plane Residual Stresses}

\subsubsection{Results}

Figures 4.2 and 4.3 show the in-plane residual stresses in Test 2 after the part has cooled fully. The stress distribution is similar for all other test cases and the maximum and minimum stresses occur in the same, or adjacent, elements in all of the test cases. It should be noted that Hodge et al. [17] used a similar mesh density for a geometrically simpler part and found that the model over-predicted the residual stresses; therefore, the following results will be interpreted from a qualitative point of view.

The maximum value for the stress in the X-direction $\left(\sigma_{x x}\right)$ occurs in the middle of the unsupported section just above the down-skin surface. The minimum $\sigma_{x x}$ occurs on the up-skin surface above the first support tooth adjacent to the unsupported overhang section. The average tensile stress in the lower portion of the unsupported overhang section is $\sigma_{x x} \approx 525[\mathrm{MPa}]$, and the average compressive stress in the upper portion is $\sigma_{x x} \approx-225[\mathrm{MPa}]$, .

The maximum and minimum stresses in the Y-direction occur in the same location as their X-direction counterparts. The average tensile stress in the lower portion of the unsupported overhang section is $\sigma_{y y} \approx 225[\mathrm{MPa}]$, and the average compressive stress in the upper portion is $\sigma_{y y} \approx-125[\mathrm{MPa}]$, .

The contour ranges in Figures 4.2 and 4.3 are truncated to $-200[\mathrm{MPa}$ ] to 200

$[\mathrm{MPa}]$, for visual comparison and to highlight the stress distribution throughout the part. 

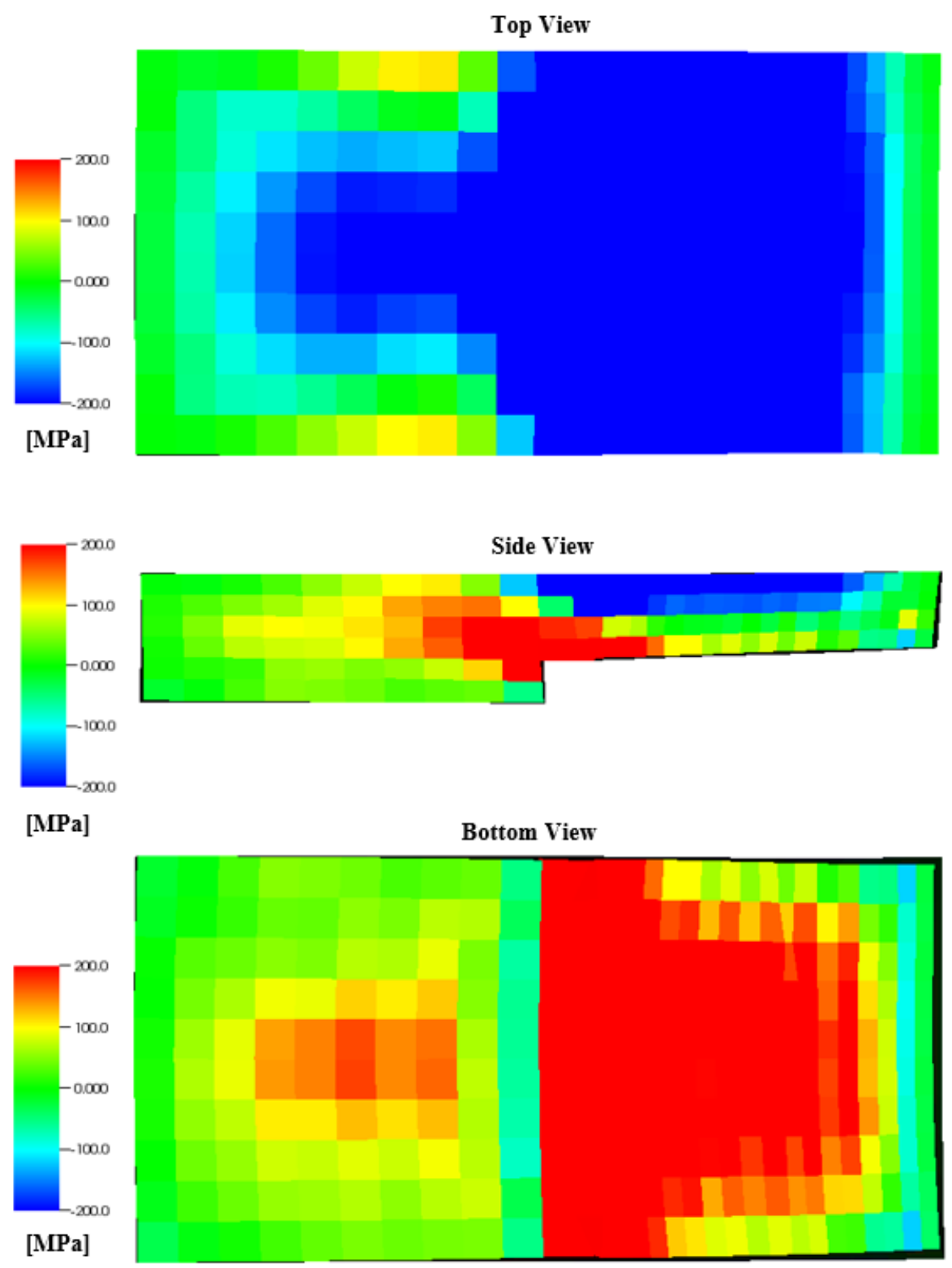

Figure 4.2 - Test $1 \sigma_{x x}$ 

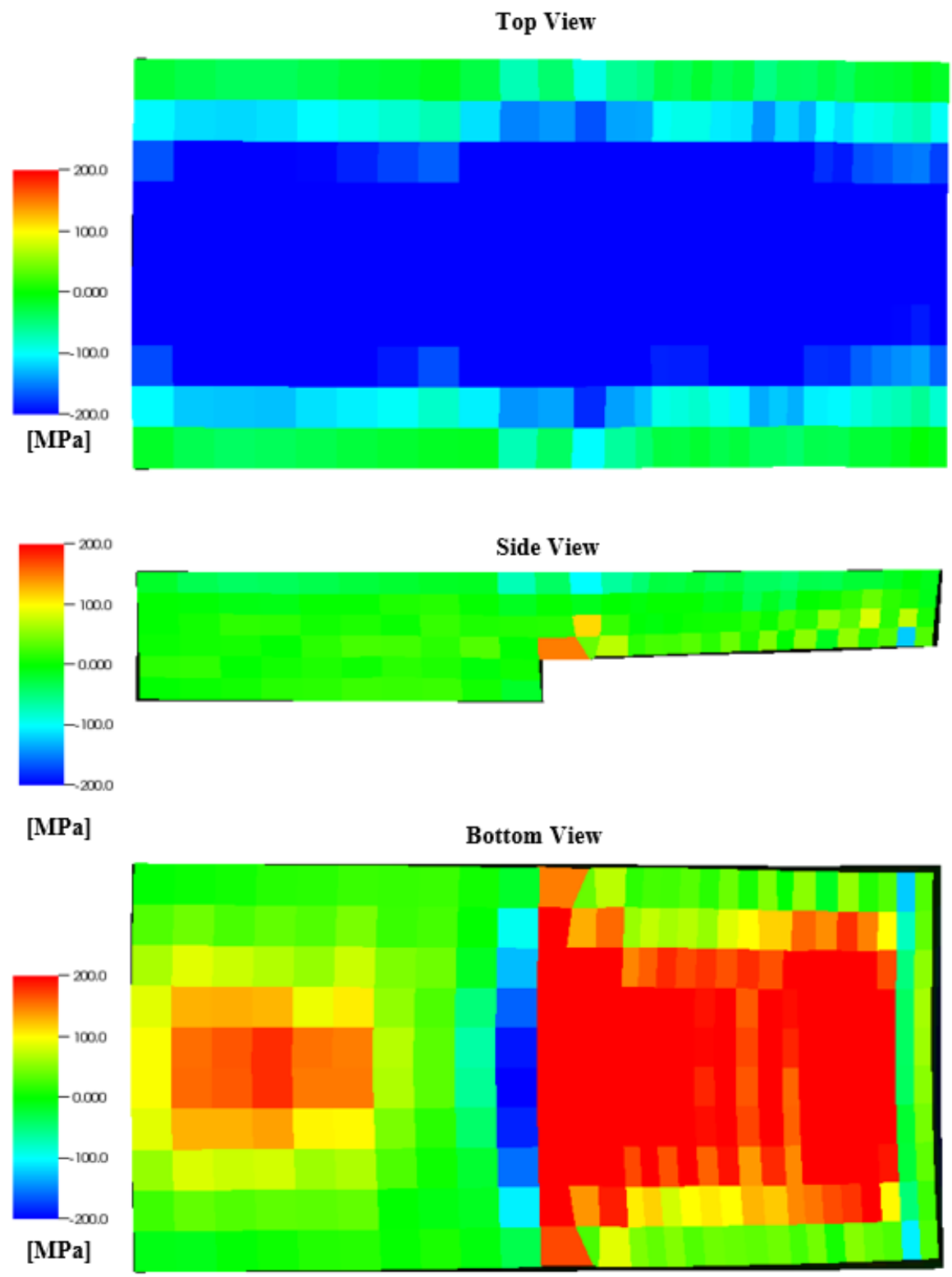

Figure 4.3 - Test $1 \sigma_{y y}$

\subsubsection{Discussion}

Figure 4.4 shows the in-plane residual stress distribution in the upper and lower regions of the part. $\sigma_{x x, l o w e r}$ is the stress along a line from point $(11,15,12.5)$ to point $(16,15,12.5)$ and $\sigma_{x x, \text { upper }}$ is the stress along a line from point $(11,15,14.5)$ to 
point $(16,15,14.5) . \sigma_{y y, l o w e r}$ is the stress along a line from point $(15,11,12.5)$ to point $(15,19,12.5)$ and $\sigma_{x x, u p p e r}$ is the stress along a line from point $(15,11,14.5)$ to point $(15,19,14.5)$. In the lower region the maximum stress occurs in the middle of the part, and in the upper region the minimum stress occurs in the middle of the part. This stress distribution would result in a "peeling away" effect discussed by Wu et al. [58].

Additionally, the magnitude of the residual stresses in the Y-direction are larger than the residual stresses in the X-direction. While $\sigma_{x x}$ is larger than $\sigma_{y y}$ in the unsupported overhang section, the residual stress magnitudes are larger in the Ydirection everywhere else. For example, in the layer $(14[\mathrm{~mm}] \leq Z \leq 14.5[\mathrm{~mm}])$ of Test $1, \sigma_{x x, a v g}=-29.9[\mathrm{MPa}]$ and $\sigma_{y y, a v g}=-99.1[\mathrm{MPa}]$. This result suggests that residual stress parallel to the scan vector, $\sigma_{y y}$, are typically larger in magnitude than the residual stress perpendicular to the scan vector, $\sigma_{x x}$, which agrees with the literature.

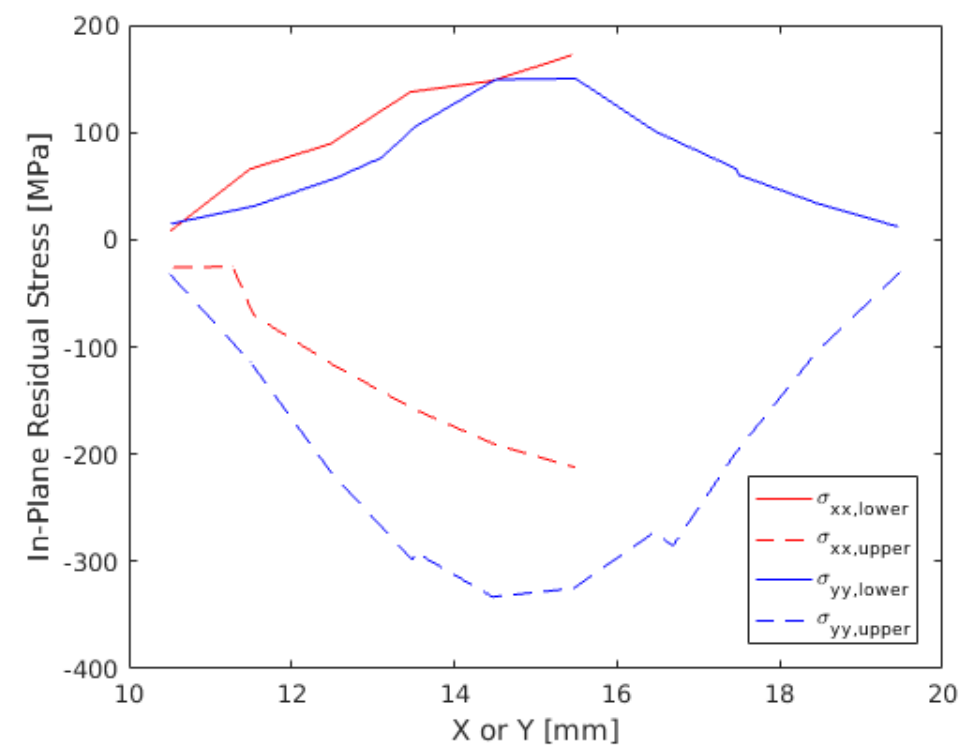

Figure 4.4 - In-Plane Residual Stress Distribution 


\subsection{Axial Residual Stresses}

\subsubsection{Results}

Figure 4.5 shows the axial residual stress in Test 2 after the part has cooled fully. Like the in-plane residual stresses, the axial stress is similar in all four test cases. The maximum tensile stress also occurs in the unsupported overhang section and has an average value of $\sigma_{z z} \approx 175[\mathrm{MPa}]$. The maximum compressive stress occurs on the down-skin surface just above the last tooth at the end of the overhang; however, large compressive residual stresses are also present in the edge of the part at $X \approx 19[\mathrm{~mm}]$. In the compressed region of the part near the beginning of the overhang feature, the average axial residual stress is $\sigma_{z z} \approx-100[\mathrm{MPa}]$. 

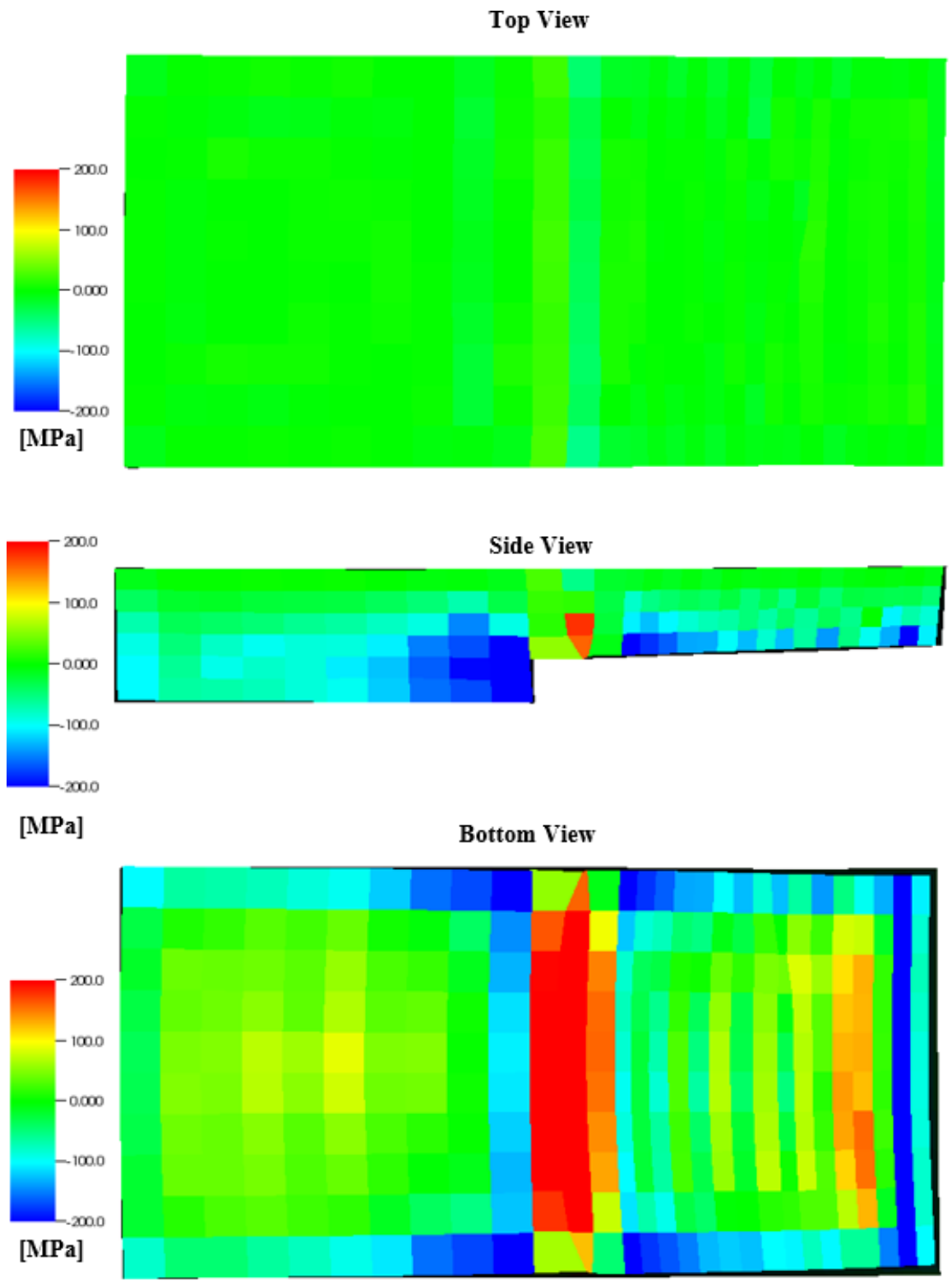

Figure 4.5 - Test $1 \sigma_{z z}$

\subsubsection{Discussion}

Contrary to the results, an axial stress distribution with high tensile stress in the outer region of the part and compressive stress in the center of the part was expected. The disagreement between the model results and the literature is a result of the particular 
global scanning strategy used in the model. Unlike the scan strategy used by Hodge et al. [17], the perimeter of the part was solidified before the interior of the part and the scan speed was lower. As a result of the particular scan strategy, the interior elements solidified after the exterior elements. Additionally, larger thermal gradients between the interior and exterior elements developed during each successive layer addition.

Figures 4.6 and 4.7 show the temperature contour over a section of the part as the layer $(12.5[\mathrm{~mm}] \leq Z \leq 13[\mathrm{~mm}])$ is solidified. At $t=82.9[\mathrm{~s}]$, both elements A and B are molten, shown in Figure 4.8. At $t=83.6[\mathrm{~s}]$, element A has solidified, while element $\mathrm{B}$ is still molten. After $t \approx 85[\mathrm{~s}]$, both elements are solid and continue to cool down to the ambient temperature. The difference in solidification time introduces residual stress in the two elements via the molten layer cool-down mechanism which is illustrated in Figure 4.9.

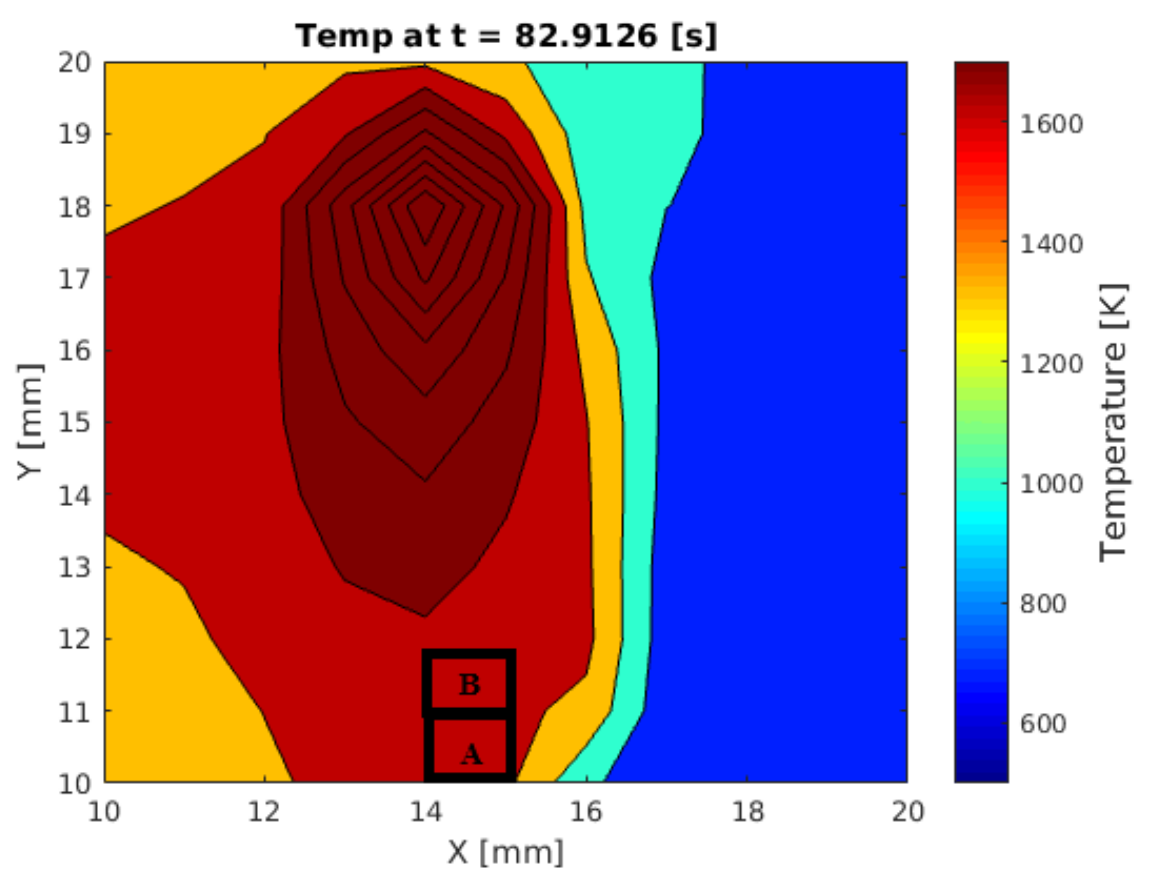

Figure 4.6 - Part Temperature Contour 1 


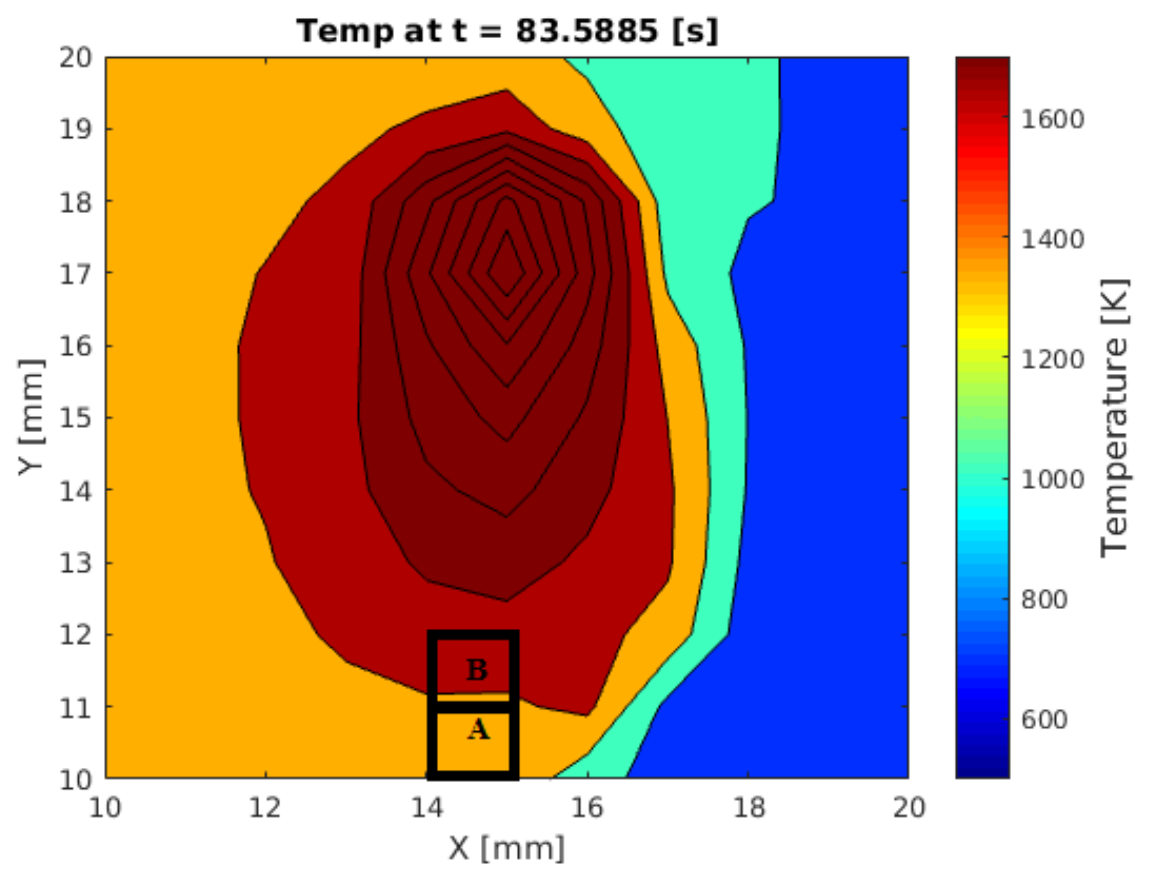

Figure 4.7 - Part Temperature Contour 2

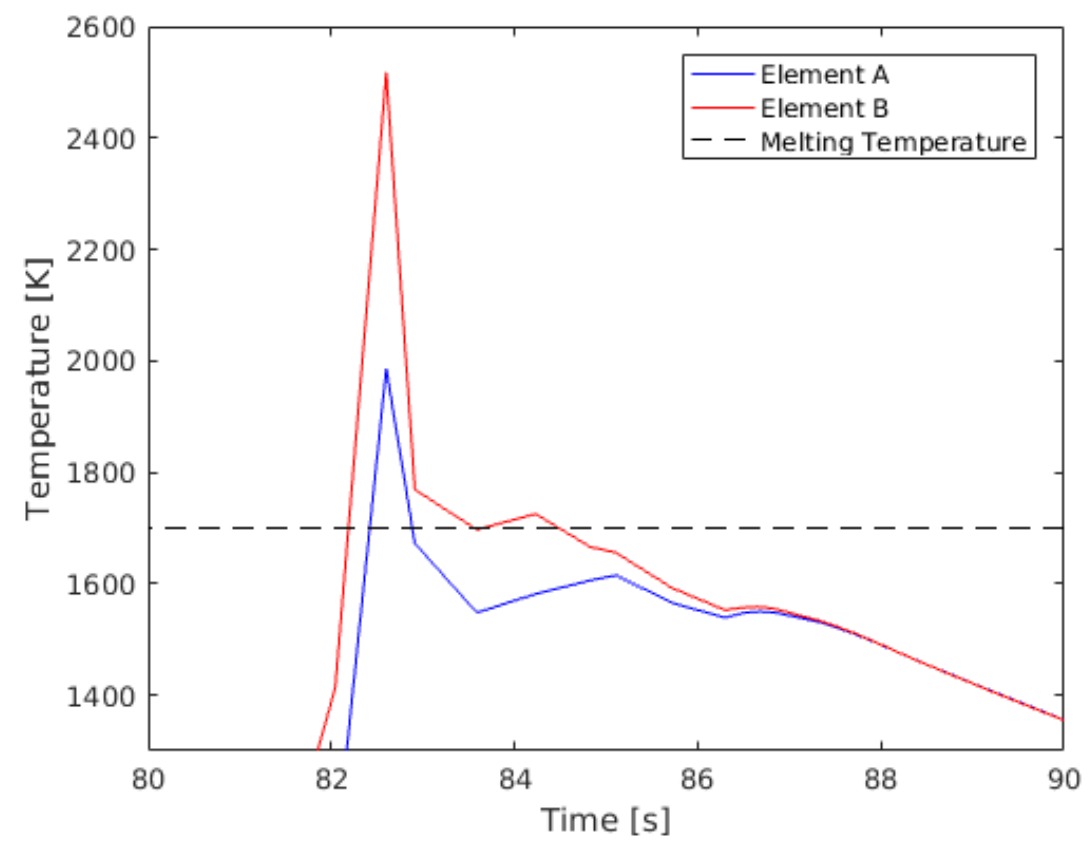

Figure 4.8 - Temperature in Elements A and B 
When element A solidifies, it is at height $Z_{A, S}$ and temperature $T_{m}$, the melting temperature. After element A solidifies, it continues to contract as it's temperature drops below $T_{m}$. When element $\mathrm{B}$ solidifies it is at the height $Z_{B, S}$ and temperature $T_{m}$. Once both elements are solid, they quickly reach an equilibrium temperature and cool-down to the ambient temperature. Since both elements are the same material, the thermal contraction from melting to ambient temperature that would result in a stress-free state is the same; however, the starting heights, $Z_{A, S}$ and $Z_{B, S}$, are different. As a result the elements $\mathrm{A}$ and $\mathrm{B}$ reach an equilibrium height $Z_{f}$ at $T_{a m b}$. The final height is less than $Z_{A, 0}$ and greater than $Z_{B, 0}$ which are the stress-free heights for elements A and B, respectively. The resulting strain in elements A and B apply a tensile load to element A and a compressive load to element B. An example of the molten layer cool-down mechanism is shown in Figure 4.10, where the initial tensile load in the solidified outer layer, element A, is reduced from approximately 20 $[\mathrm{MPa}]$ to $-4[\mathrm{MPa}]$. 

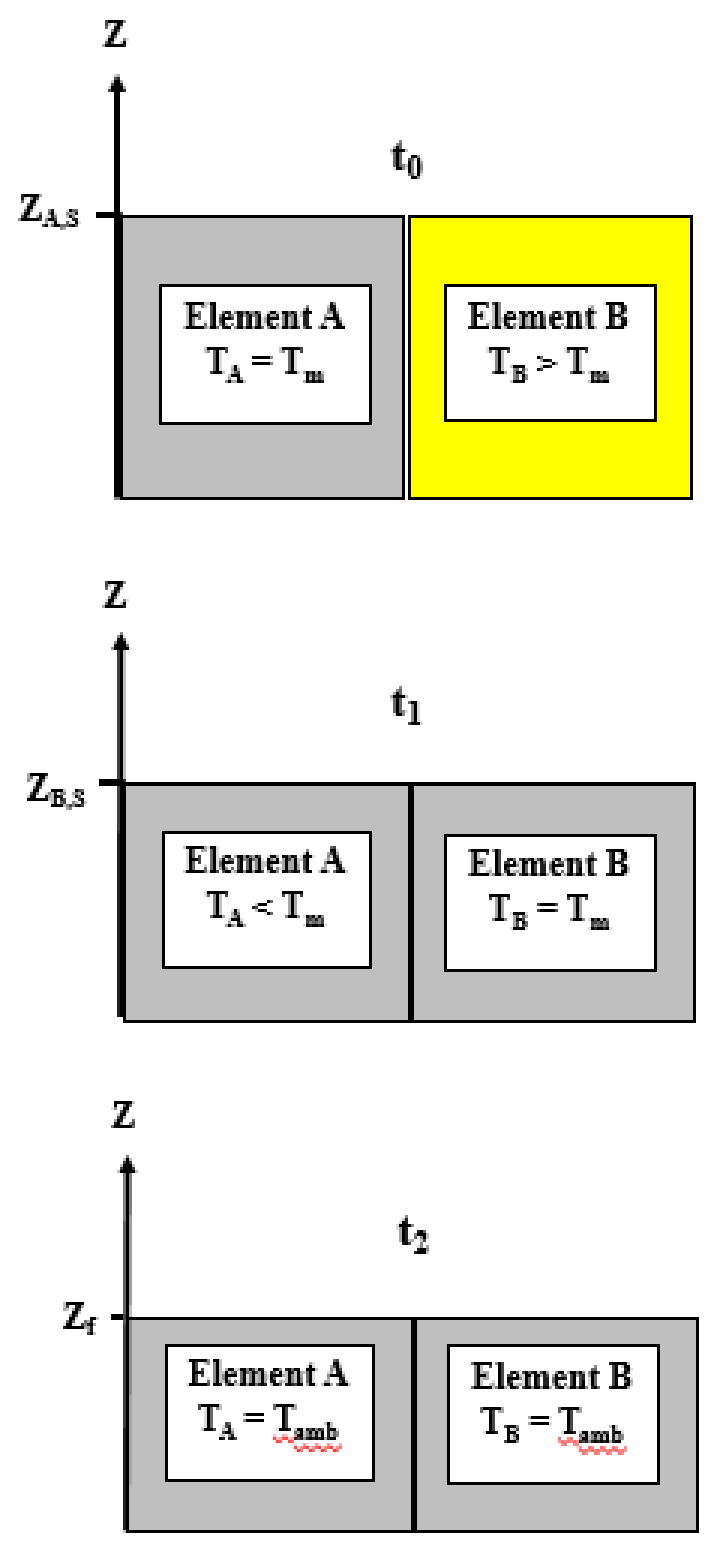

Figure 4.9 - 1D Illustration of the Molten Layer Cool-Down Mechanism

Figure 4.10 shows the axial residual stress in elements $\mathrm{A}$ and $\mathrm{B}$ throughout the entire simulation starting at $t=80.0[\mathrm{~s}]$ when the layer is added to the model. It should be noted that the stress profile was sampled every 10 seconds in the simulation while the element temperature was calculated from nodal values sampled every 100 timesteps, typically every 0.5 seconds during heating. Despite the large stress 
sampling timestep, Figure 4.10 shows that over time the stress in element A decrease while the stress in element B increases. The change in residual stresses over time are a result of the TGM. When a new layer is added, a larger temperature difference is produced between elements A and B. The expansion of element B is restricted by element A, and the higher temperature of element B results in a lower yield stress. Thus, element B is plastically deformed during each layer addition. Returning to the 1D example, the plastic deformation in element B effectively reduces the stress-free height $Z_{B, 0}$, which results in higher compressive loads in element $\mathrm{A}$ and higher tensile loads in element B.

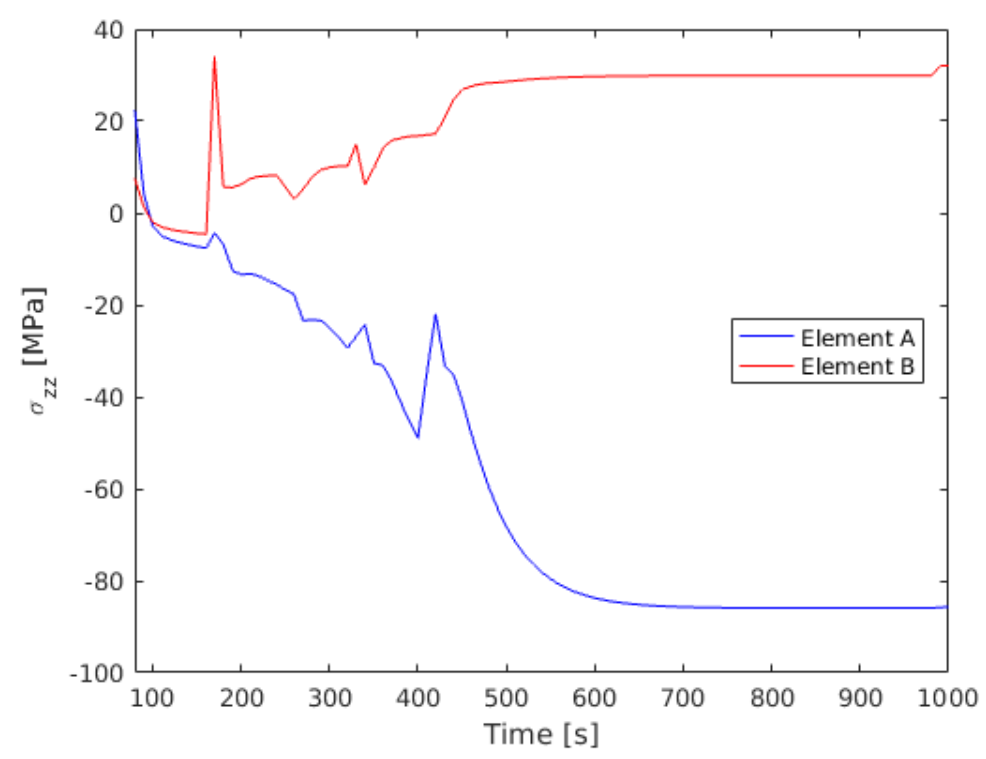

Figure 4.10 - Axial Residual Stress in Elements A and B

There is a similar effect in the unsupported section of the overhang, where the cooling rates are much lower than the adjacent elements. Figure 4.11 shows the temperature gradient that results from the reduced cooling rates in the unsupported overhang section. The elements in the unsupported overhang section cool down slower than the adjacent elements which results in the large tensile residual stress above the unsupported overhang region and the large compressive stress in the part adjacent to 
the unsupported overhang region. The same mechanism results in the large compressive stress in the elements above the support structure teeth and the tensile stress in the adjacent unsupported elements.

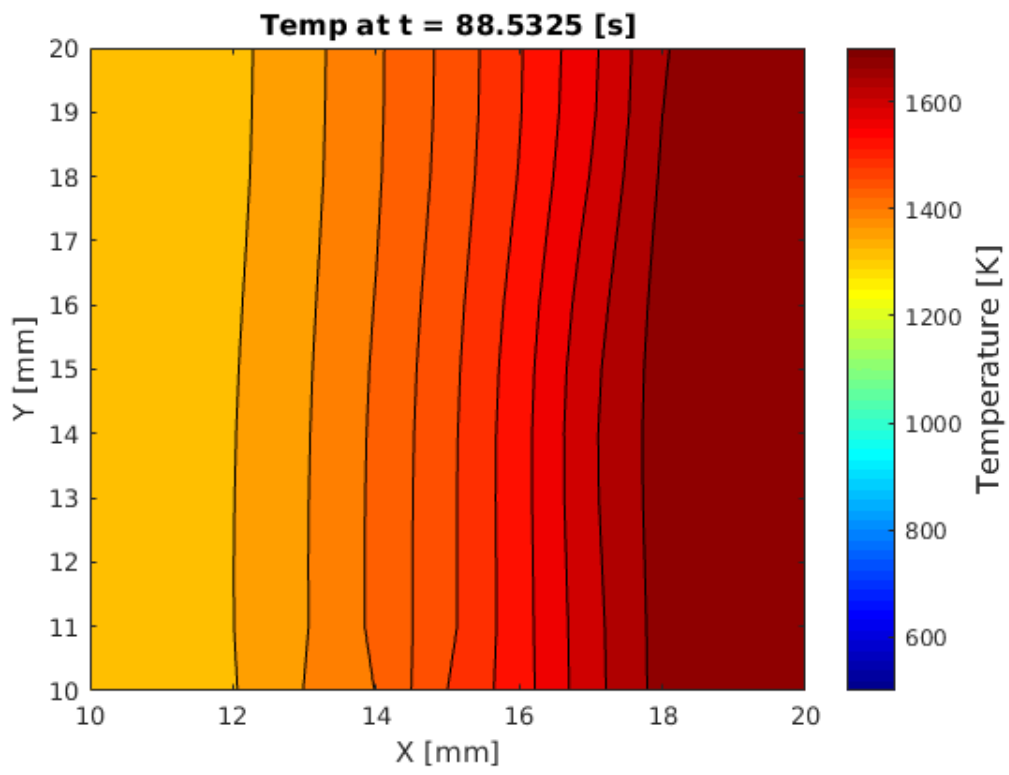

Figure 4.11 - Part Temperature Contour 3

\subsection{Effect of Contact Area Parameters}

\subsubsection{Results}

The maximum and minimum values for all of the residual stresses are tabulated in Table 4.1. The table also includes the total contact area between the overhang downskin surface and the tops of the support structure teeth. 
Table 4.1 - Factorial Study Maximum Residual Stress Results

\begin{tabular}{|c|c|c|c|c|c|c|c|}
\cline { 2 - 8 } \multicolumn{1}{c|}{} & Contact Area & \multicolumn{2}{c|}{$\sigma_{x x}[\mathrm{MPa}]$} & \multicolumn{2}{c|}{$\sigma_{y y}[\mathrm{MPa}]$} & \multicolumn{2}{c|}{$\sigma_{z z}[\mathrm{MPa}]$} \\
\cline { 2 - 8 } \multicolumn{1}{c|}{} & {$\left[\mathrm{mm}^{2}\right]$} & Max & Min & Max & Min & Max & Min \\
\hline Test 2 & 13.5 & 860 & -560 & 463 & -404 & 324 & -365 \\
\hline Test 1 & 27.0 & 872 & -483 & 445 & -363 & 269 & -297 \\
\hline Test 4 & 31.5 & 912 & -532 & 487 & -380 & 278 & -296 \\
\hline Test 3 & 63.0 & 936 & -526 & 479 & -372 & 308 & -237 \\
\hline
\end{tabular}

Figures 4.12 - 4.17 show the effects of varying the Top Length and Tooth Spacing on the residual stresses. All of the stress values are normalized to the maximum residual stress, the compressive stress in the X-direction. The only definitive correlation that can be drawn from the Figures 4.12 and 4.13 is that the Top Length has an adverse effect on the tensile stress in the X-direction, most notably at the unsupported section of the overhanging feature.
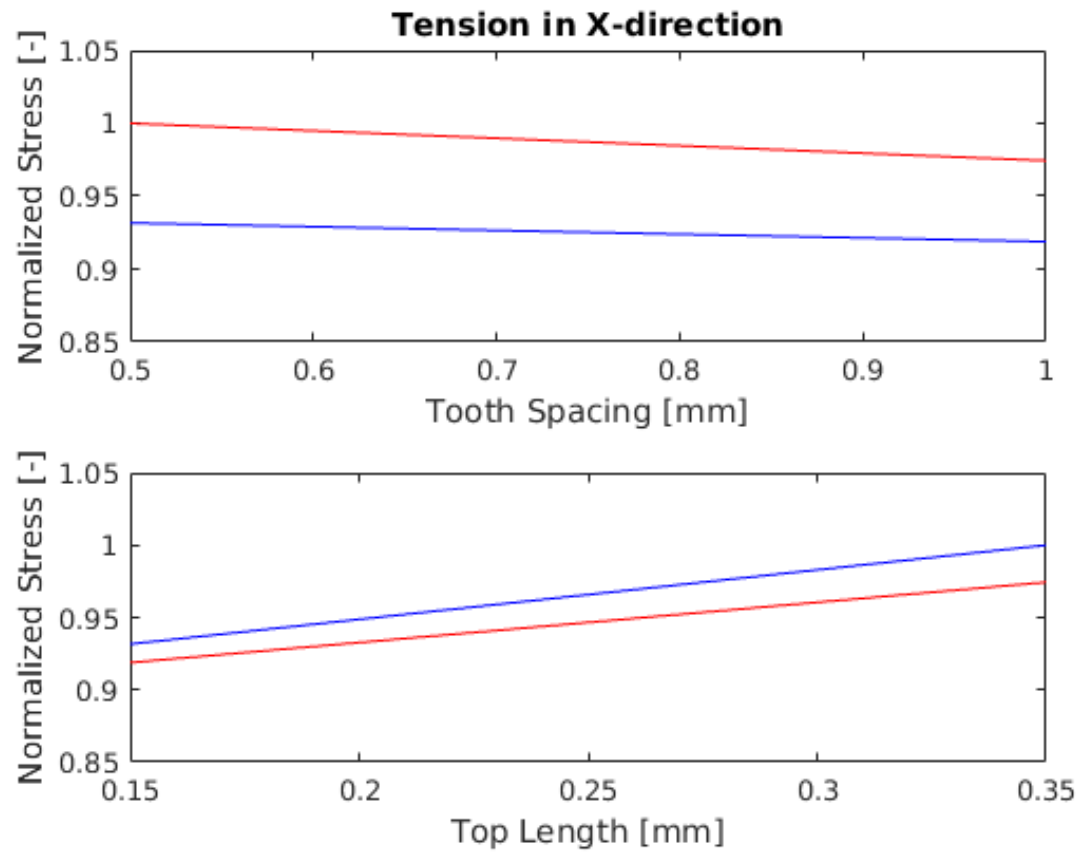

Figure 4.12 - Top Length and Tooth Spacing Effect on Tensile $\sigma_{x x}$ 

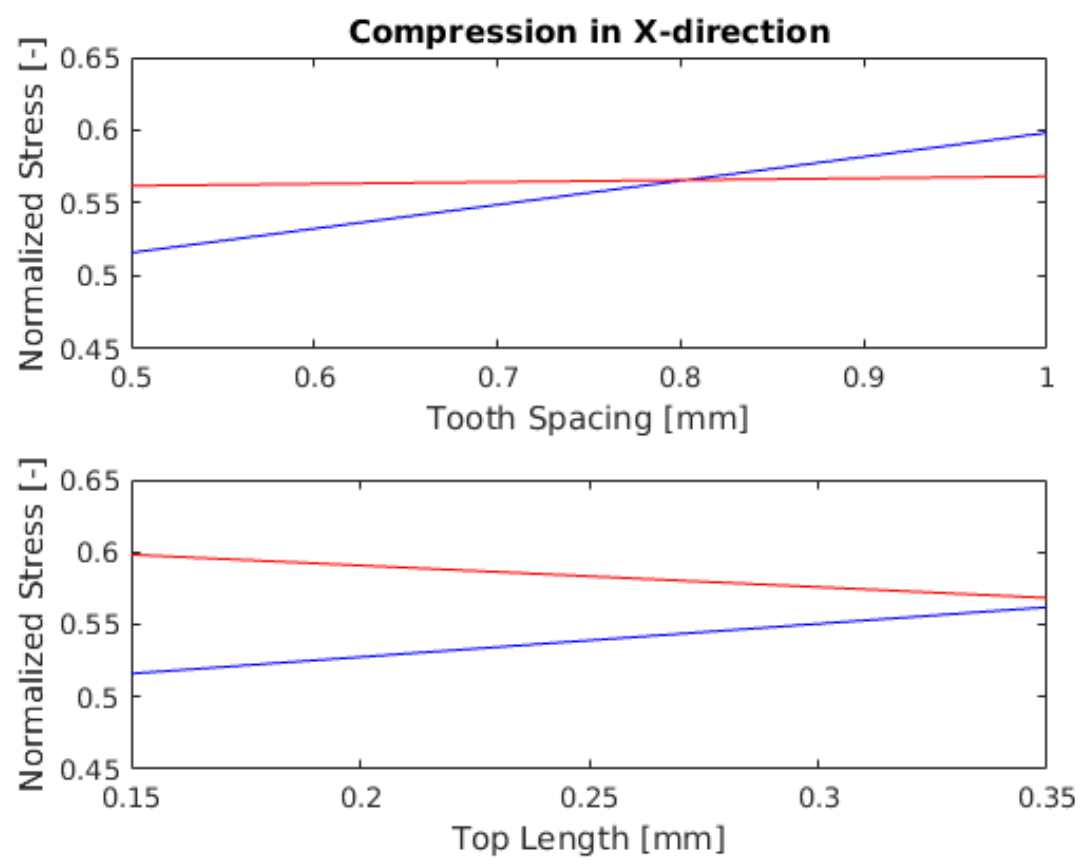

Figure 4.13 - Top Length and Tooth Spacing Effect on Compressive $\sigma_{x x}$

Figures 4.14 and 4.15 show the effects of varying the Top Length and Tooth Spacing on the residual stresses in the Y-direction. Again, the only definitive correlation is the inverse effect between the Top Length and the tensile residual stress. 

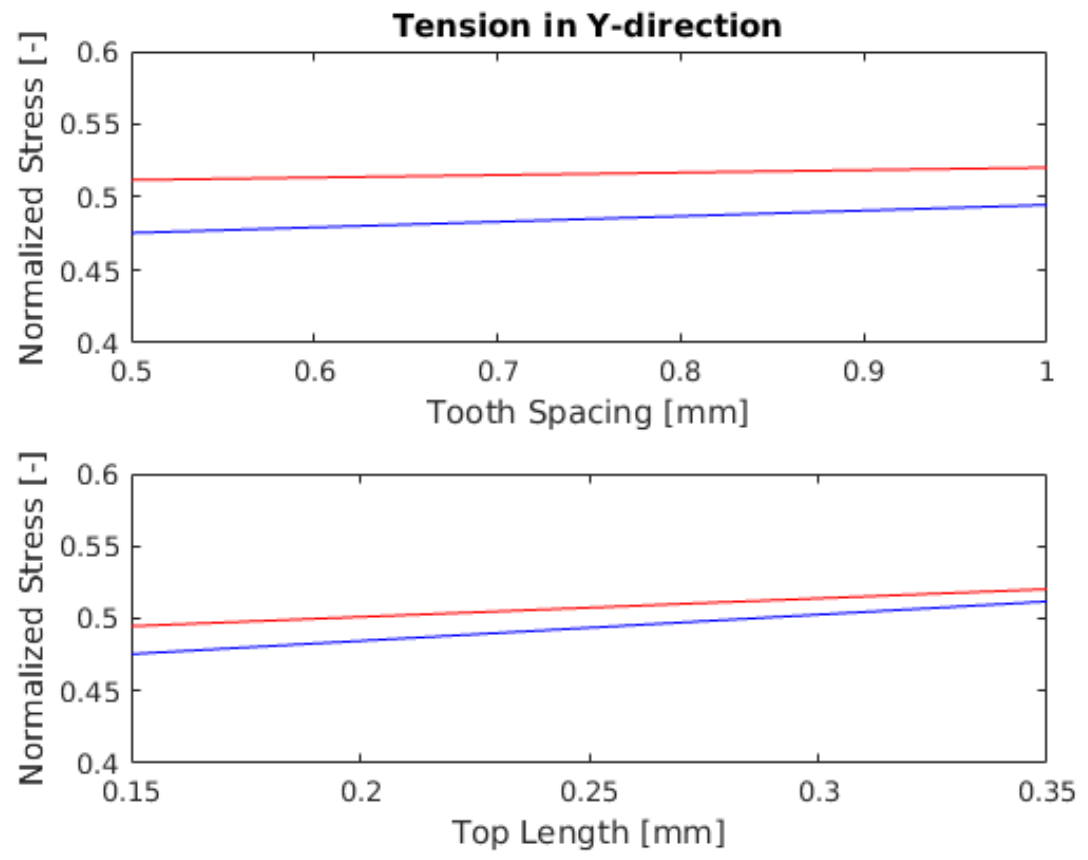

Figure 4.14 - Top Length and Tooth Spacing Effect on Tensile $\sigma_{y y}$
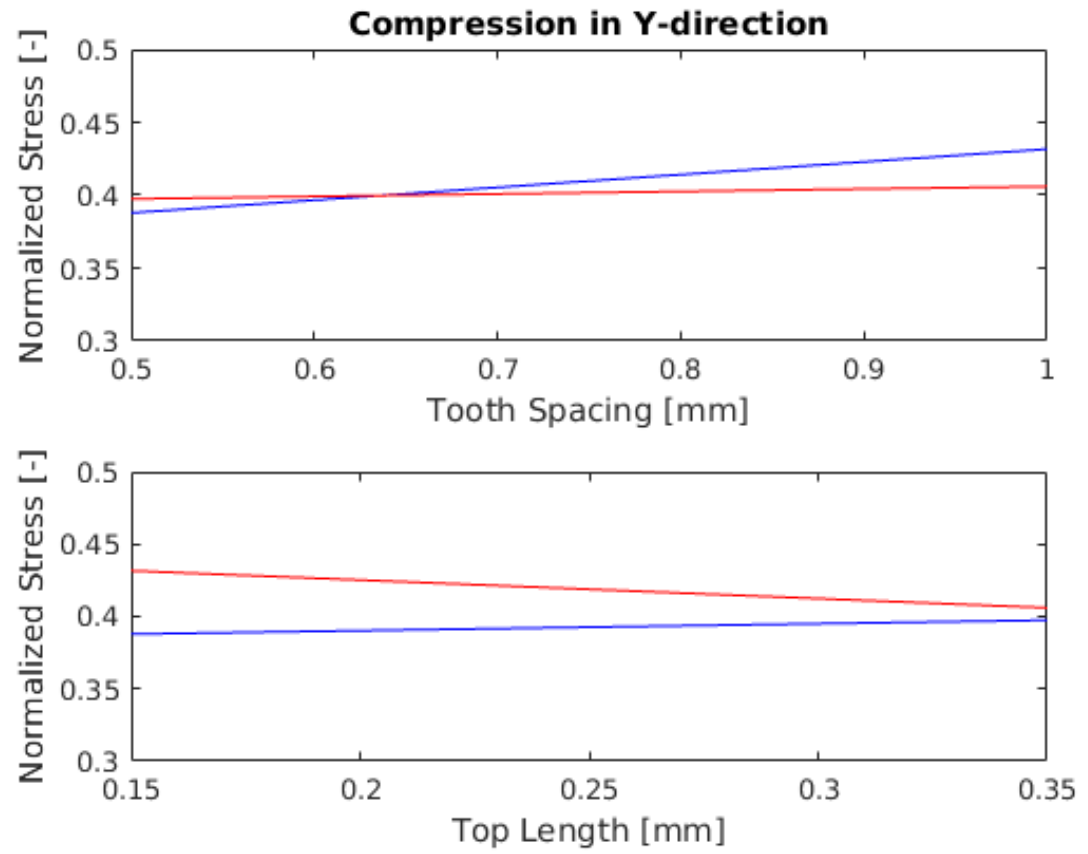

Figure 4.15 - Top Length and Tooth Spacing Effect on Compressive $\sigma_{y y}$ 
It can be seen in Figure 4.17 that increasing the Top Length reduces the magnitude of the compressive stress in the Z-direction. Figure 4.17 also shows the correlation between Tooth Spacing and the compressive stress in the Z-direction. Reducing the spacing between the contact points results in lower residual stress in the overhanging feature.
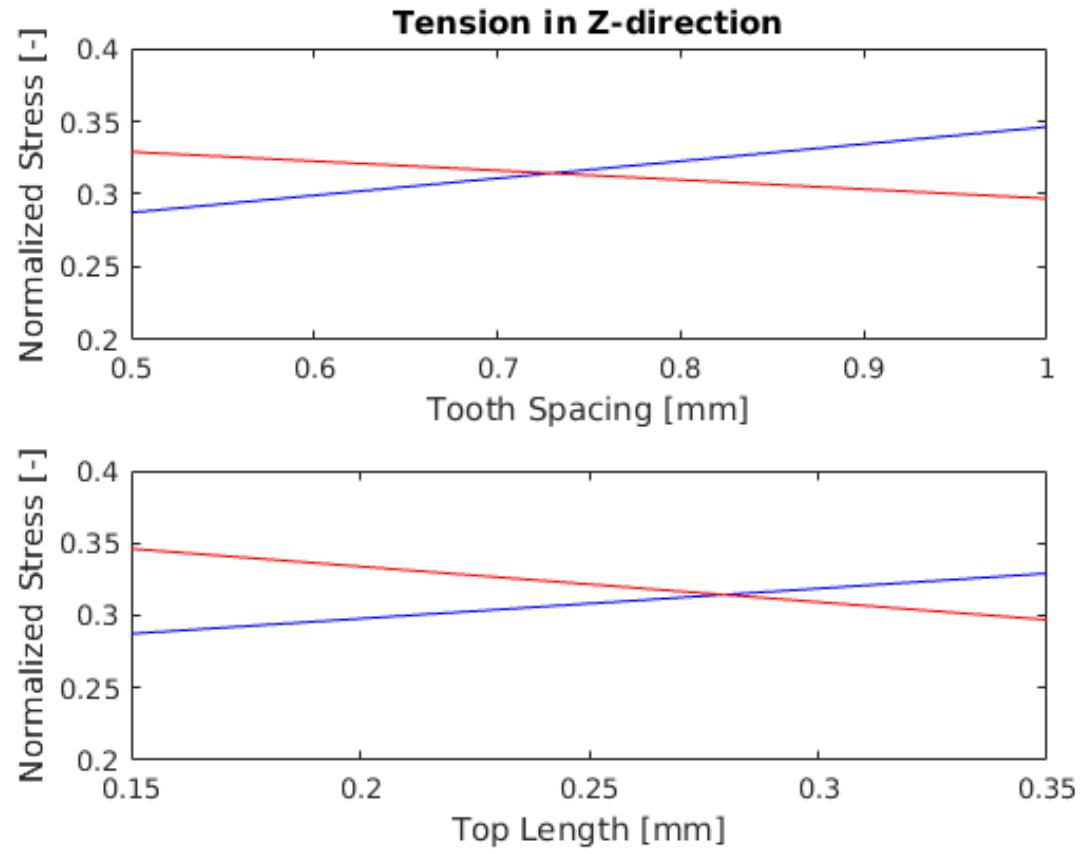

Figure 4.16 - Top Length and Tooth Length Effect on Tensile $\sigma_{z z}$ 

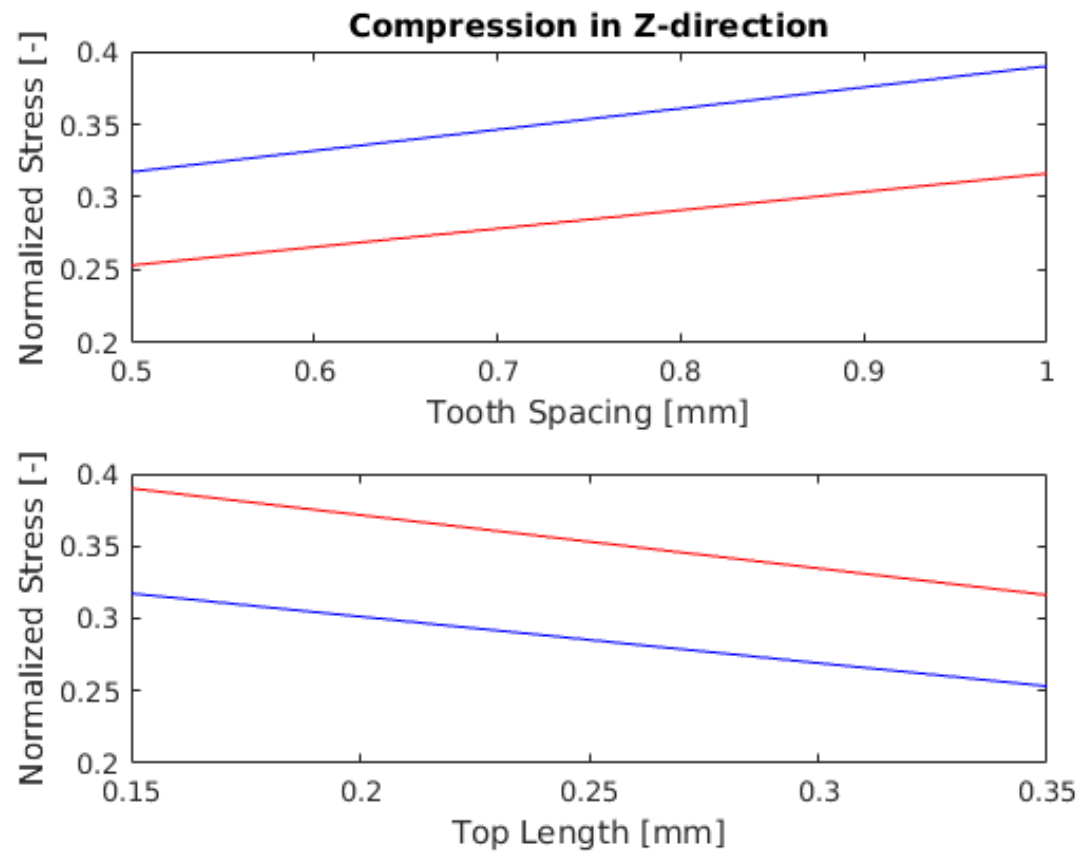

Figure 4.17 - Top Length and Tooth Length Effect on Compressive $\sigma_{z z}$

\subsubsection{Discussion}

From the parameter interaction plots above, it can be concluded that increasing the Top Length reduces the axial compressive residual stresses and increases the in-plane tensile residual stresses while the Tooth Spacing only has a noteworthy effect on the

compressive axial residual stress. Since the Top Length and the Tooth Spacing are proportional to the total contact area between the overhang and the support structure, the extreme values of the residual stresses are plotted against the total contact area in Figure 4.18. Figure 4.18 shows the relative magnitudes of the residual stresses in each direction and Figure 4.19 shows the same data normalized with the maximum values. The normalized data shows that only the maximum compressive stress in the Z-direction and the maximum tensile stress in the X-direction correlate to the total contact area well. 


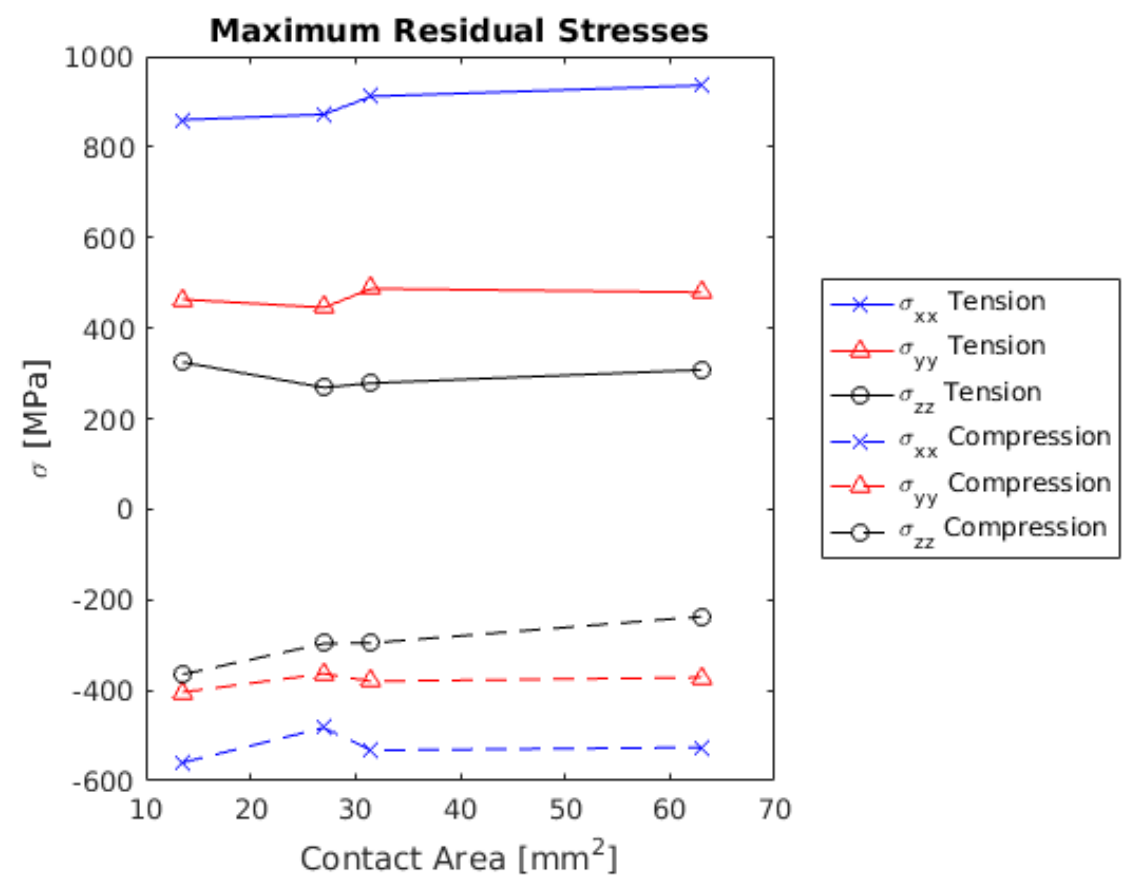

Figure 4.18 - Maximum Tensile and Minimum Compressive Residual Stresses

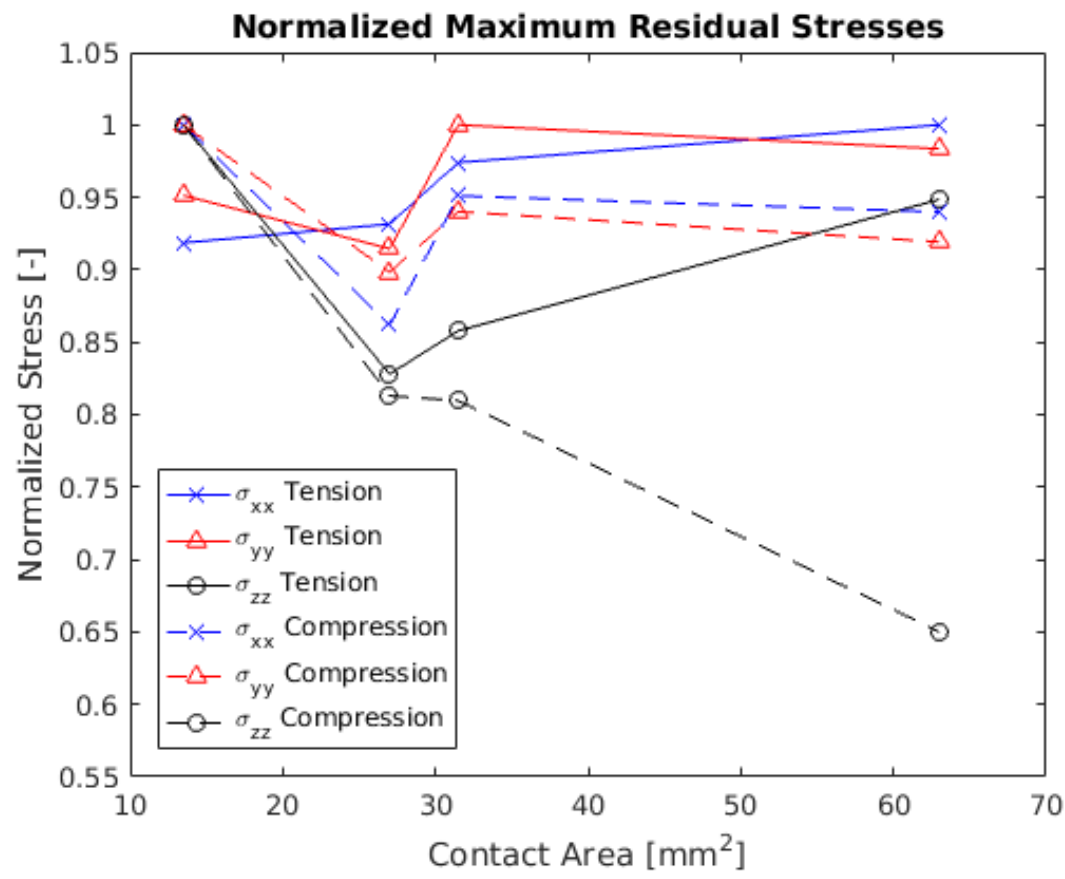

Figure 4.19 - Normalized Maximum Tensile and Minimum Compressive Residual Stresses 
The maximum compressive stress in the Z-direction and the maximum tensile stress in the X-direction are both a result of large temperature gradients in the overhang area. By increasing the contact area, the average cooling rate in the overhang area is increased, which decreases the compressive stress in the Z-direction. Increasing the average cooling rate in the overhang causes the overhang area adjacent to the unsupported section to solidify faster, which increases the maximum tensile stress in the X-direction over the unsupported section. Therefore, increasing contact area increases the tensile stress in the unsupported overhang section between the part and the support structure.

These findings are related by the fact that reducing the cooling rate gradient, the variation in maximum cooling rates across the build plane, reduces the magnitude of the resulting residual stresses. 


\title{
CHAPTER 5
}

\author{
Conclusion
}

\subsection{Summary}

The goal of this research was to investigate the effects of two contact area parameters, the tooth spacing and top length, on the residual stresses in overhanging features. To achieve this goal, a thermo-mechanical model of the SLM process was implemented in Diablo, a multi-physics finite element code developed by LLNL. The thermal model, which accounted for phase change from powder to consolidated material, was coupled to the mechanical model using a staggered scheme. The bulk material, 316 stainless steel, was modeled as either powder or consolidated elastic-plastic material with temperature dependent material properties and isotropic work hardening. To reduce the computational cost, a global heating strategy and volume effective material properties were used in the model. Deflections predicted by the model were compared to experimentally observed deflections and the resulting residual stresses were compared to results in the literature. While there were discrepancies in the residual stress distribution in some regions the experimental validation showed a reasonable agreement between the trend and relative magnitude of the deflections. Additionally, the source of the residual stress distribution discrepancies can be explained by examining the global scanning strategy.

The results of the $2^{2}$ factorial study are summarized below:

1. The tooth spacing only effects the compressive axial residual stress. 
2. Increasing the top length reduced the magnitude of the compressive axial residual stresses and increased the magnitude of the tensile in-plane residual stresses.

3. In general, reducing the in-plane cooling rate gradients reduces residual stresses.

\subsection{Future Work}

The SLM process is relatively new and it is still in development, therefore it provides many research opportunities. While it has been addressed by some studies, there is still a need for research pertaining to residual stresses in overhanging features which could lead to general support structure design guidelines. Additionally, part-scale models of the SLM process provide insight that is difficult to gain from experiments; however, there are a limited number of part-scale models. The following topics should be considered for future work:

1. The effect of reducing in-plane cooling rate gradients,

2. Additional studies on the effects of support structure parameters such as contact spacing, top length, tooth height, and tooth base interval,

3. Novel support structures, such as the heat sink proposed by [6],

4. Experimental validation of the use of volume effective material properties,

5. Improved global scanning strategy. 


\section{BIBLIOGRAPHY}

[1] ASTM International. Standard terminology for additive manufacturing technologies : designation F2792-12a. ASTM International, West Conshohocken, PA, 2012.

[2] Lourdes D. Bobbio, Shipin Qin, Alexander Dunbar, Panagiotis Michaleris, and Allison M. Beese. Characterization of the strength of support structures used in powder bed fusion additive manufacturing of Ti-6al-4v. Additive Manufacturing, 14:60-68, March 2017.

[3] F. Brückner, D. Lepski, and E. Beyer. Modeling the Influence of Process Parameters and Additional Heat Sources on Residual Stresses in Laser Cladding. Journal of Thermal Spray Technology, 16(3):355-373, 2007.

[4] F. Calignano. Design optimization of supports for overhanging structures in aluminum and titanium alloys by selective laser melting. Materials $\& 6$ Design, 64:203-213, December 2014.

[5] Luke N. Carter, Christopher Martin, Philip J. Withers, and Moataz M. Attallah. The influence of the laser scan strategy on grain structure and cracking behaviour in SLM powder-bed fabricated nickel superalloy. Journal of Alloys and Compounds, 615:338-347, December 2014.

[6] Bo Cheng and Kevin Chou. Thermal Stresses Associated with Part Overhang Geometry in Electron Beam Additive Manufacturing: Process Parameter Effects. In Proc. Annu. Int. Solid Freeform Fabr. Symp, volume 25, page 1076, 2014. 
[7] Bo Cheng and Kevin Chou. Geometric consideration of support structures in part overhang fabrications by electron beam additive manufacturing. ComputerAided Design, 69:102-111, December 2015.

[8] M. Cloots, A. B. Spierings, and K. Wegener. Assessing new support minimizing strategies for the additive manufacturing technology SLM. In 24th International SFF Symposium-An Additive Manufacturing Conference, Austin, USA, University of Texas at Austin, pages 631-643, 2013.

[9] N Contuzzi, S.L. Campanelli, and A.D. Ludovico. 3d Finite Element Analysis in the Selective Laser Melting Process. Int. J. Simul. Model, 10:113-121, 2011.

[10] Erik R. Denlinger, Jarred C. Heigel, Pan Michaleris, and T.A. Palmer. Effect of inter-layer dwell time on distortion and residual stress in additive manufacturing of titanium and nickel alloys. Journal of Materials Processing Tech., 215(Complete):123-131, 2015.

[11] T.W. Eagar and N.S. Tasi. Temperature fields produced by traveling distributed heat sources. Welding Research Supplement, pages 346-355.

[12] C. H. Fu and Y. B. Guo. Three-Dimensional Temperature Gradient Mechanism in Selective Laser Melting of Ti-6al-4v. Journal of Manufacturing Science and Engineering, 136(6):061004-061004-7, October 2014.

[13] M.X. Gan and C.H. Wong. Practical support structures for selective laser melting. Journal of Materials Processing Technology, 238:474-484, December 2016.

[14] Rishi Ganeriwala. Example Diablo SLM Model Input Deck, 2017.

[15] A. V. Gusarov, I. Yadroitsev, Ph. Bertrand, and I. Smurov. Model of Radiation and Heat Transfer in Laser-Powder Interaction Zone at Selective Laser Melting. Journal of Heat Transfer, 131(7):072101, 2009. 
[16] N. E. Hodge, R. M. Ferencz, and J. M. Solberg. Implementation of a thermomechanical model for the simulation of selective laser melting. Computational Mechanics, 54(1):33-51, July 2014.

[17] N.E. Hodge, R.M. Ferencz, and R.M. Vignes. Experimental comparison of residual stresses for a thermomechanical model for the simulation of selective laser melting. Additive Manufacturing, 12:159-168, October 2016.

[18] Henry Hu and Stavros Argyropoulos. Mathematical modelling of solidification and melting: a review. Modelling and simulation in Material Science and Engineering, 4:371-396, 1996.

[19] Y. Huang, L.J. Yang, X.Z. Du, and Y.P. Yang. Finite element analysis of thermal behavior of metal powder during selective laser melting. International Journal of Thermal Sciences, 104:146-157, June 2016.

[20] Ahmed Hussein, Yan Chunze, Richard Everson, and Liang Hao. Innovative developments in virtual and physical prototyping: proceedings of the 5th International Conference on Advanced Research and Rapid Prototyping, Leiria, Portugal, 28 September - 1 October, 2011 ; the 5th International Conference on Advanced Research in Virtual and Physical Prototyping (VRP 2011)]. CRC Press/Balkema, Boca Raton, Fla., 2012. OCLC: 839002766.

[21] Ahmed Hussein, Liang Hao, Chunze Yan, and Richard Everson. Finite element simulation of the temperature and stress fields in single layers built withoutsupport in selective laser melting. Materials \& Design (1980-2015), 52:638-647, December 2013.

[22] Ahmed Hussein, Liang Hao, Chunze Yan, Richard Everson, and Philippe Young. Advanced lattice support structures for metal additive manufacturing. Journal of Materials Processing Technology, 213(7):1019-1026, July 2013. 
[23] Jamasp Jhabvala, Eric Boillat, Cdric Andr, and Rmy Glardon. An innovative method to build support structures with a pulsed laser in the selective laser melting process. The International Journal of Advanced Manufacturing Technology, 59(1-4):137-142, March 2012.

[24] Jukka-Pekka Jrvinen, Ville Matilainen, Xiaoyun Li, Heidi Piili, Antti Salminen, Ismo Mkel, and Olli Nyrhil. Characterization of Effect of Support Structures in Laser Additive Manufacturing of Stainless Steel. Physics Procedia, 56:72-81, 2014.

[25] C. R. Knowles, T. H. Becker, and R. B. Tait. Residual stress measurements and structural integrity implications for selective laser melted Ti-6al-4v. South African Journal of Industrial Engineering, 23(3):119-129, 2012.

[26] T. A. Krol, M.F. Zaeh, and C. Seidel. Modellierung transienter Effekte in der Struktursimulation von Schichtbauverfahren. Number Band 246 in Forschungsberichte IWB. Herbert Utz Verlag, Mnchen, 2011.

[27] T.A. Krol, C. Seidel, and M.F. Zaeh. Prioritization of Process Parameters for an Efficient Optimisation of Additive Manufacturing by Means of a Finite Element Method. Procedia CIRP, 12:169-174, 2013.

[28] J.-P. Kruth, J. Deckers, E. Yasa, and R. Wauthle. Assessing and comparing influencing factors of residual stresses in selective laser melting using a novel analysis method. Proceedings of the Institution of Mechanical Engineers, Part B: Journal of Engineering Manufacture, 226(6):980-991, June 2012.

[29] Erdem Kundakcioglu, Ismail Lazoglu, and Suraj Rawal. Transient thermal modeling of laser-based additive manufacturing for $3 \mathrm{~d}$ freeform structures. The International Journal of Advanced Manufacturing Technology, 85(1):493-501, 2016. 
[30] M. Labudovic, D. Hu, and R. Kovacevic. A three dimensional model for direct laser metal powder deposition and rapid prototyping. Journal of Materials Science, 38(1):35-49, 2003.

[31] C. Li, J.F. Liu, and Y.B. Guo. Prediction of Residual Stress and Part Distortion in Selective Laser Melting. Procedia CIRP, 45:171-174, 2016.

[32] JunChang Li, C. Langlade, and A.B. Vannes. Evaluation of the thermal field developed during pulsed laser treatments: semi analytical calculation. Surface and Coatings Technology, 115:87-93, 1999.

[33] Yang Liu, Yongqiang Yang, and Di Wang. A study on the residual stress during selective laser melting (SLM) of metallic powder. The International Journal of Advanced Manufacturing Technology, 87(1-4):647-656, October 2016.

[34] Daryl Logan. A First Course in the Finite Element Method. Brooks/Cole, Pacific Grove, CA, USA, 3rd edition, 2002.

[35] V. Manvatkar, A. De, and T. DebRoy. Spatial variation of melt pool geometry, peak temperature and solidification parameters during laser assisted additive manufacturing process. Materials Science and Technology, 31(8):924-930, 2015.

[36] M. Matsumoto, M. Shiomi, K. Osakada, and F. Abe. Finite element analysis of single layer forming on metallic powder bed in rapid prototyping by selective laser processing. International Journal of Machine Tools and Manufacture, 42(1):61$67,2002$.

[37] W. Meiners, K. Wissenbach, and A. Gasser. Selective laser sintering at melting temperature. Google Patents, April 2001. 
[38] Peter Mercelis and JeanPierre Kruth. Residual stresses in selective laser sintering and selective laser melting. Rapid Prototyping Journal, 12(5):254-265, October 2006.

[39] Raya Mertens, Stijn Clijsters, Karolien Kempen, and Jean-Pierre Kruth. Optimization of Scan Strategies in Selective Laser Melting of Aluminum Parts With Downfacing Areas. Journal of Manufacturing Science and Engineering, 136(6):061012, October 2014.

[40] Albert E. Patterson, Sherri L. Messimer, and Phillip A. Farrington. Overhanging Features and the SLM/DMLS Residual Stresses Problem: Review and Future Research Need. Technologies, 5(2), 2017.

[41] Haiko Pohl, Abdolreza Simchi, Munther Issa, and H. Calefi Dias. Thermal stresses in direct metal laser sintering. In Proceedings of the 12th Solid Freeform Fabrication Symposium, Austin, TX, 2001.

[42] O. Poyraz, E. Yasa, G. Akbulut, A. Orhangul, and S. Pilatin. Investigation of support structures for direct metal laser sintering (DMLS) of IN625 parts. In Proceedings of Solid Freeform Fabrication (SFF) Symposium, Austin, TX, USA, 2015.

[43] I.A. Roberts, C.J. Wang, R. Esterlein, M. Stanford, and D.J. Mynors. A threedimensional finite element analysis of the temperature field during laser melting of metal powders in additive layer manufacturing. International Journal of Machine Tools and Manufacture, 49(12-13):916-923, October 2009.

[44] Ibiye Aseibichin Roberts. Investigation of residual stresses in the laser melting of metal powders in additive layer manufacturing. 2012. 
[45] M. Shiomi, K. Osakada, K Nakamura, T Yamashita, and F. Abe. Residual Stress within Metallic Model Made by Selective Laser Melting Process. Manufacturing Technology, 53:195-198, 2004.

[46] J. M. Solberg, N. E. Hodge, R. M. Ferencz, R. A. Whitesides, M. A. Puso, I. D. Parsons, M. A. Havstad, and A. P. Wemhoff. Diablo: A parallel, implicit multiphysics finite element code for engineering analysis user manual, June 2015.

[47] M. A. Taha, A. F. Yousef, K. A. Gany, and H. A. Sabour. Selektives Laserschmelzen von hoch kohlenstoffhaltigen Sthlen: Einfluss der Abtastgeschwindigkeit und der Wrmenachbehandlung. Materialwissenschaft und Werkstofftechnik, 43(11):913-923, 2012.

[48] Lore Thijs, Frederik Verhaeghe, Tom Craeghs, Jan Van Humbeeck, and JeanPierre Kruth. A study of the microstructural evolution during selective laser melting of Ti6al4v. Acta Materialia, 58(9):3303 - 3312, 2010.

[49] Van Belle, Laurent, Boyer, Jean-Claude, and Vansteenkiste, Guillaume. Investigation of residual stresses induced during the selective laser melting process. Key. Engineering Materials, 554-557:1828-1834, 2013.

[50] Aditad Vasinonta, Jack L. Beuth, and Michelle L. Griffith. Process maps for controlling residual stress and melt pool size in laser-based SFF processes. In Solid Freeform Fabrication Proceedings, volume 8, page 206. Proc. 2000 Solid Freeform Fabrication Symposium, Austin, 2000.

[51] Pratik Vora, Kamran Mumtaz, Iain Todd, and Neil Hopkinson. AlSi12 in-situ alloy formation and residual stress reduction using anchorless selective laser melting. Additive Manufacturing, 7:12-19, July 2015.

[52] Bey Vrancken. Study of Residual Stresses in Selective Laser Melting. PhD thesis, Ku Leuven, Arenberg Doctoral School, Belgium, June 2016. 
[53] Bey Vrancken, Lore Thijs, Jean-Pierre Kruth, and Jan Van Humbeeck. Heat treatment of Ti6al4v produced by Selective Laser Melting: Microstructure and mechanical properties. Journal of Alloys and Compounds, 541:177 - 185, 2012.

[54] Di Wang, Yongqiang Yang, Ziheng Yi, and Xubin Su. Research on the fabricating quality optimization of the overhanging surface in SLM process. The International Journal of Advanced Manufacturing Technology, 65(9-12):1471-1484, April 2013.

[55] Philip J. Withers and H.K.D.H Bhadeshia. Residual Stress. Part 1: Measurement Techniques. Material Science and Technology, 17(4):355-365, 2001.

[56] Philip J. Withers and H.K.D.H Bhadeshia. Residual Stress. Part 2: Nature and Origin. Material Science and Technology, 17(4):366- 375, 2001.

[57] Spencer Wright. 3d Printing Titanium and the Bin of Broken Dreams (Part 1-3). 3D Printing Industry, 2015.

[58] Amanda S. Wu, Donald W. Brown, Mukul Kumar, Gilbert F. Gallegos, and Wayne E. King. An experimental investigation into additive manufacturinginduced residual stresses in $316 \mathrm{l}$ stainless steel. Metallurgical and Materials Transactions A, 45(13):6260-6270, 2014.

[59] I. Yadroitsev, P. Krakhmalev, I. Yadroitsava, S. Johansson, and I. Smurov. Energy input effect on morphology and microstructure of selective laser melting single track from metallic powder. Journal of Materials Processing Technology, 213(4):606-613, April 2013.

[60] Evren Yasa and Jean-Pierre Kruth. Application of laser re-melting on selective laser melting parts. Advances in Production engineering and Management, 6(4):259-270, 2011. 
[61] Michael F. Zaeh and Gregor Branner. Investigations on residual stresses and deformations in selective laser melting. Production Engineering, 4(1):35-45, February 2010 .

[62] Kai Zeng. Optimization of support structures for selective laser melting. 2015.

[63] Kai Zeng, Deepankar Pal, and Brent Stucker. A review of thermal analysis methods in Laser Sintering and Selective Laser Melting. In Proceedings of Solid Freeform Fabrication Symposium Austin, TX, 2012.

[64] Pu Zhang, Jakub Toman, Yiqi Yu, Emre Biyikli, Mesut Kirca, Markus Chmielus, and Albert C. To. Efficient Design-Optimization of Variable-Density Hexagonal Cellular Structure by Additive Manufacturing: Theory and Validation. Journal of Manufacturing Science and Engineering, 137(2):021004, April 2015.

[65] O. C. Zienkiewicz. The Finite Element Method. McGraw-Hill, Maidenhead, Berkshire Enlgand, 3rd edition, 1977.

[66] O. C. Zienkiewicz and R. L. Taylor. The Finite Element Method: For Solid and Structural Mechanics. Elsevier Butterworth Heinemann, 6th edition, 2005. 


\section{APPENDIX A}

Additional Experimental Validation Results

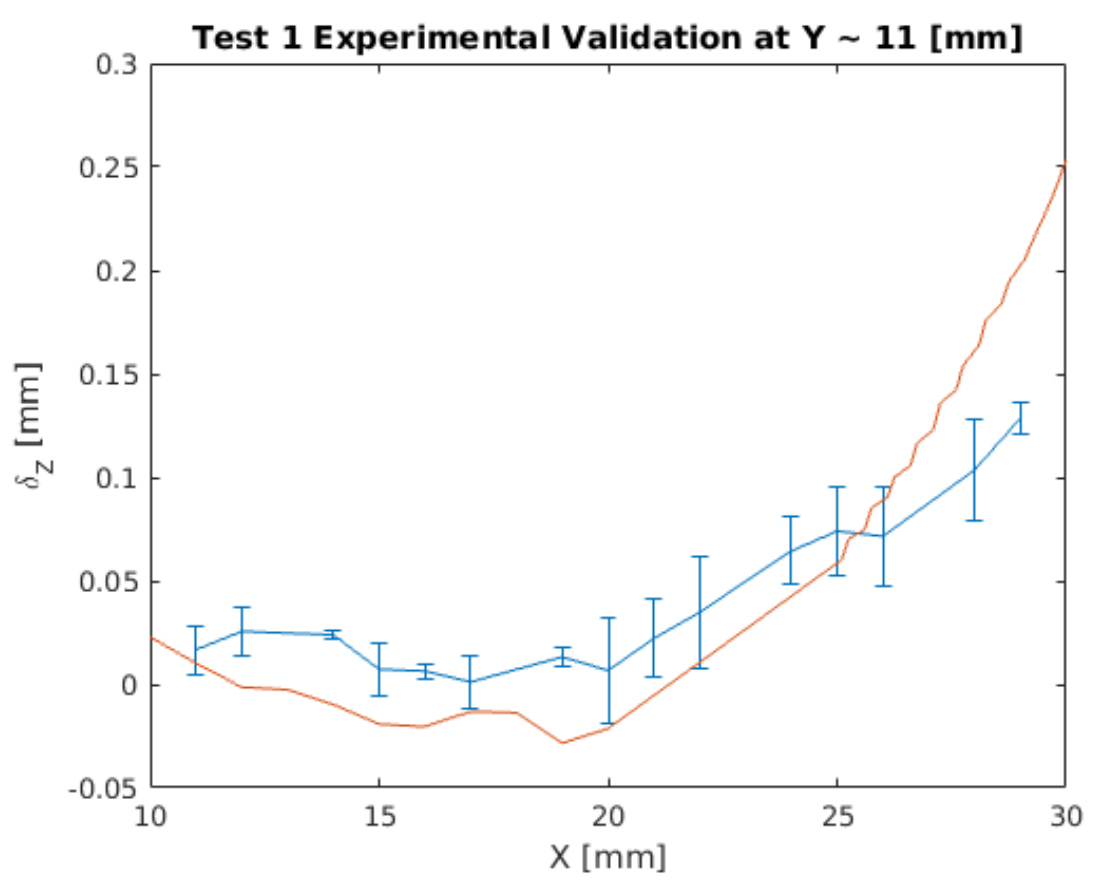

Figure A.1 - Test 1 Experimental Validation at $\mathrm{Y} \approx 11[\mathrm{~mm}]$ 


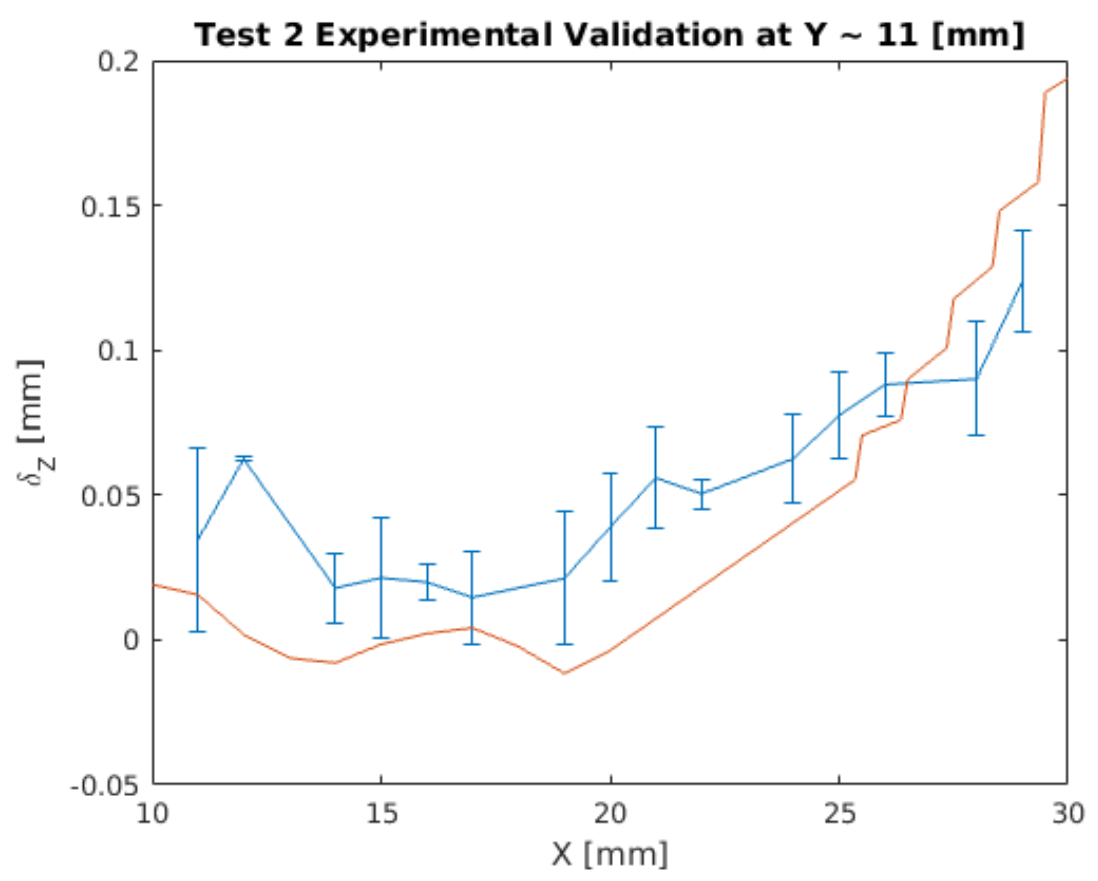

Figure A.2 - Test 2 Experimental Validation at $\mathrm{Y} \approx 11[\mathrm{~mm}]$

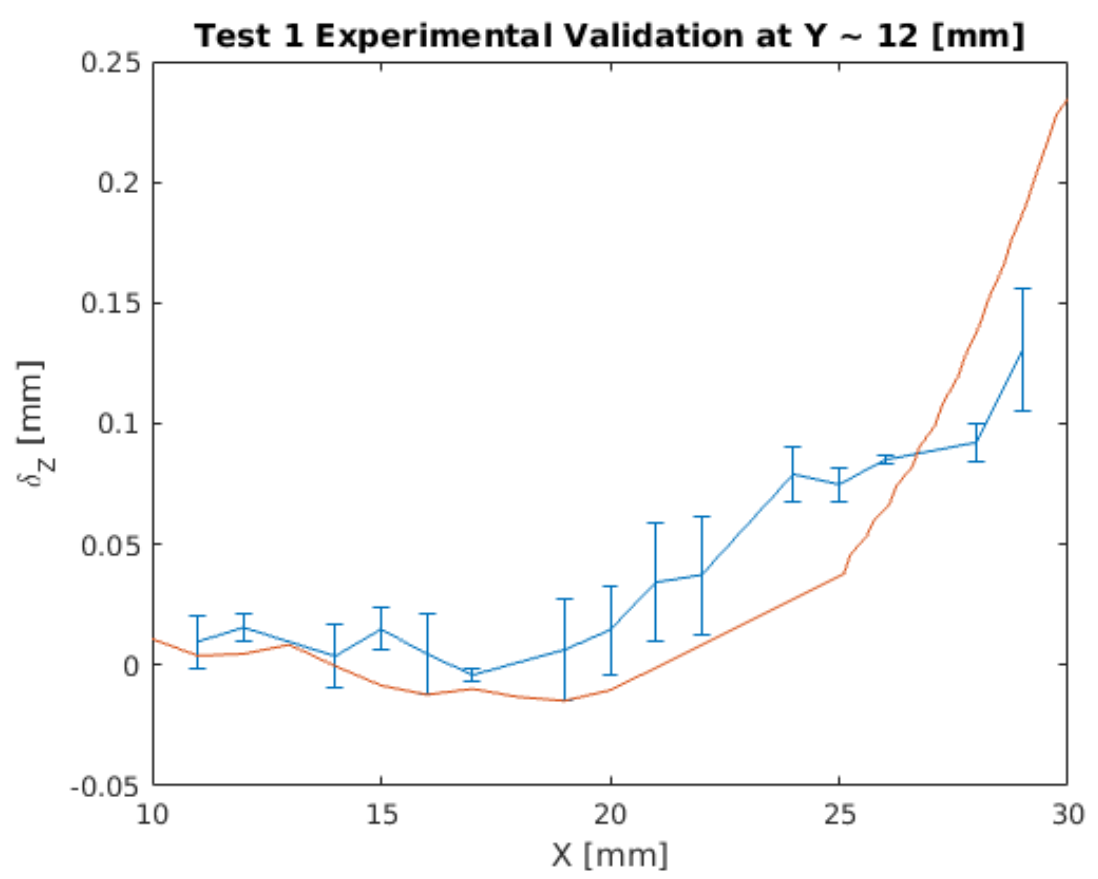

Figure A.3 - Test 1 Experimental Validation at $\mathrm{Y} \approx 12[\mathrm{~mm}]$ 


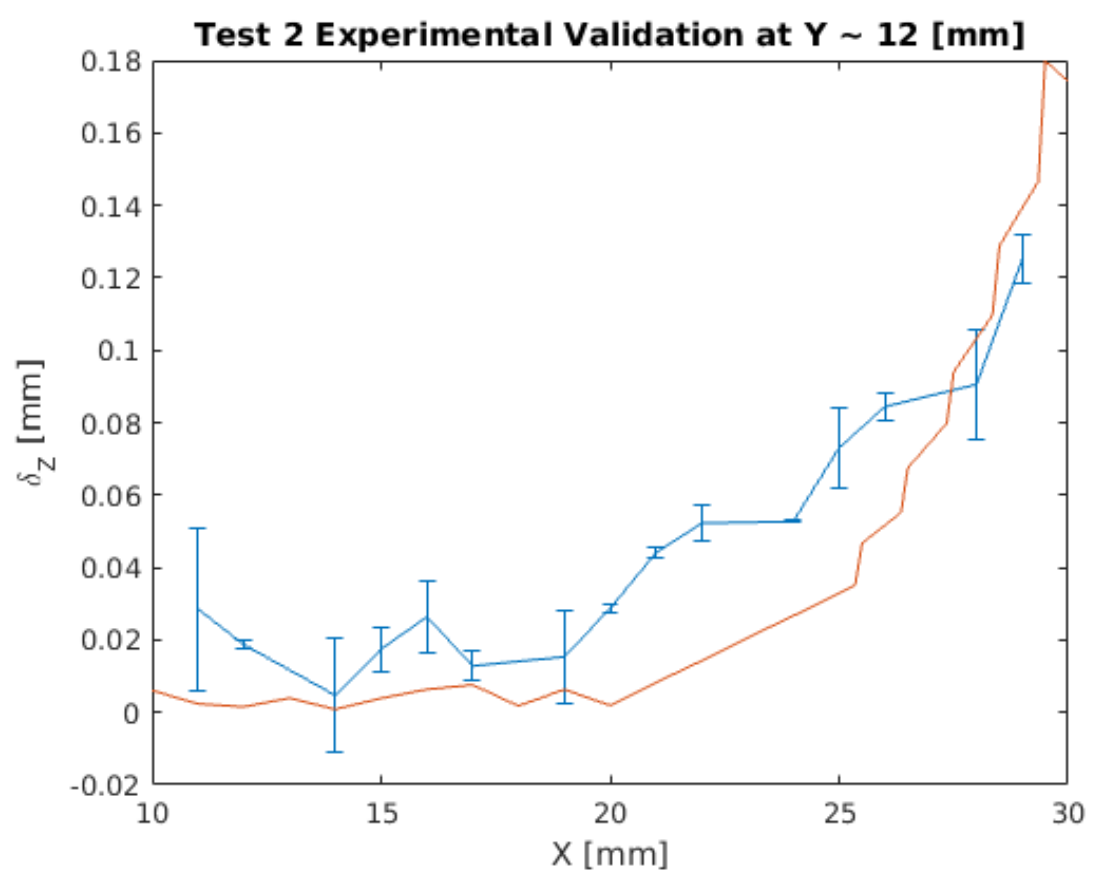

Figure A.4 - Test 2 Experimental Validation at $\mathrm{Y} \approx 12[\mathrm{~mm}]$

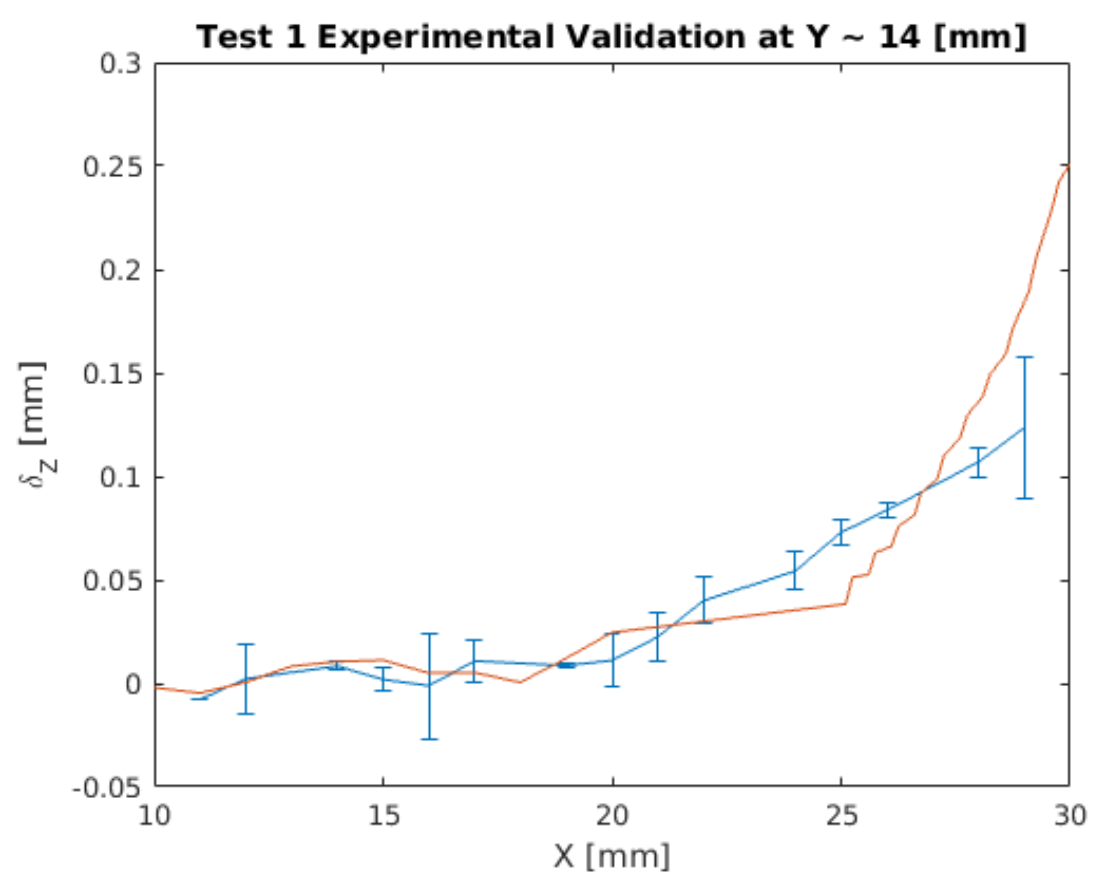

Figure A.5 - Test 1 Experimental Validation at $\mathrm{Y} \approx 14[\mathrm{~mm}]$ 


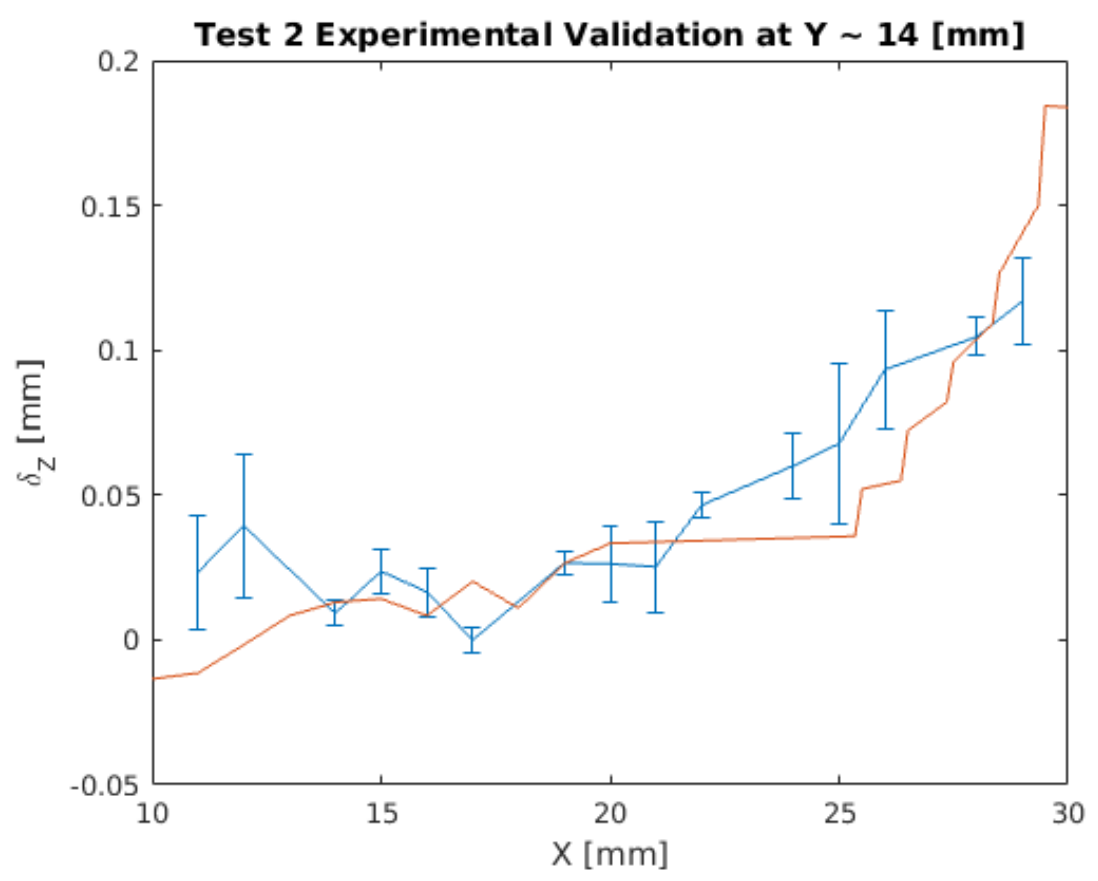

Figure A.6 - Test 2 Experimental Validation at $\mathrm{Y} \approx 14[\mathrm{~mm}]$

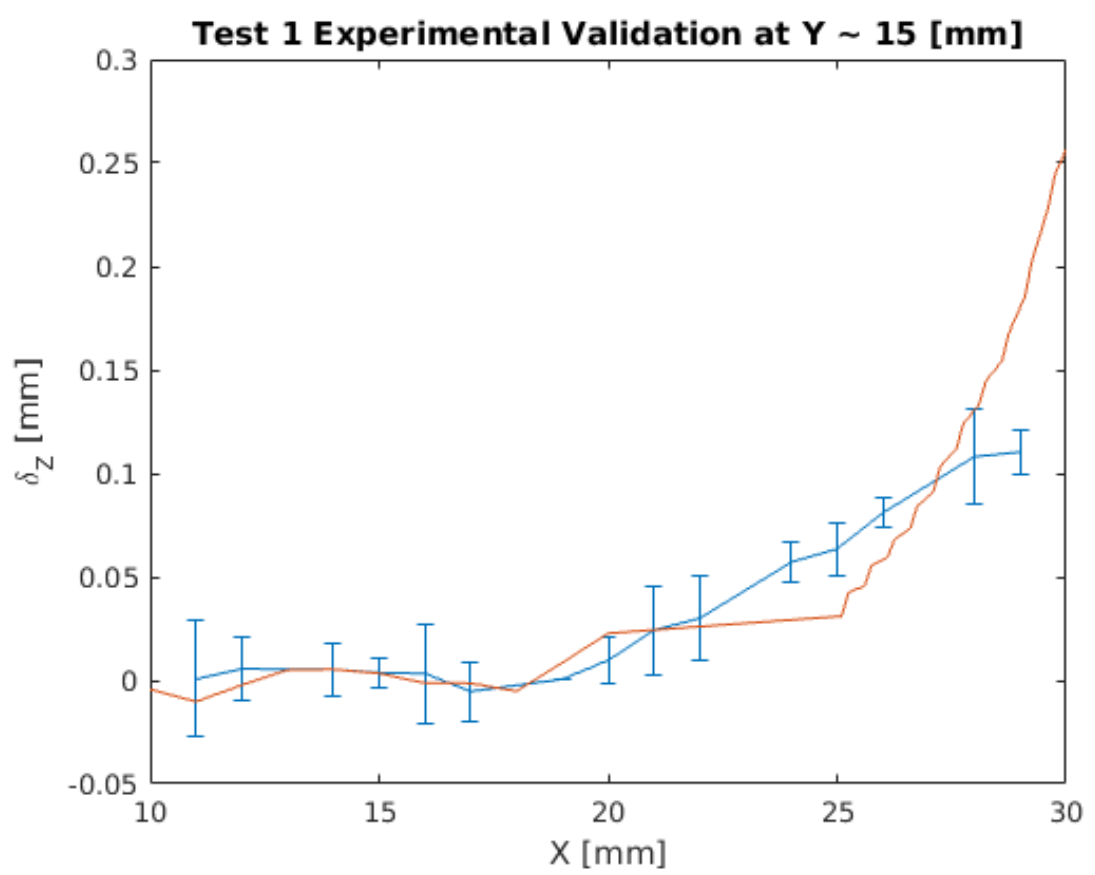

Figure A.7 - Test 1 Experimental Validation at $\mathrm{Y} \approx 15[\mathrm{~mm}]$ 


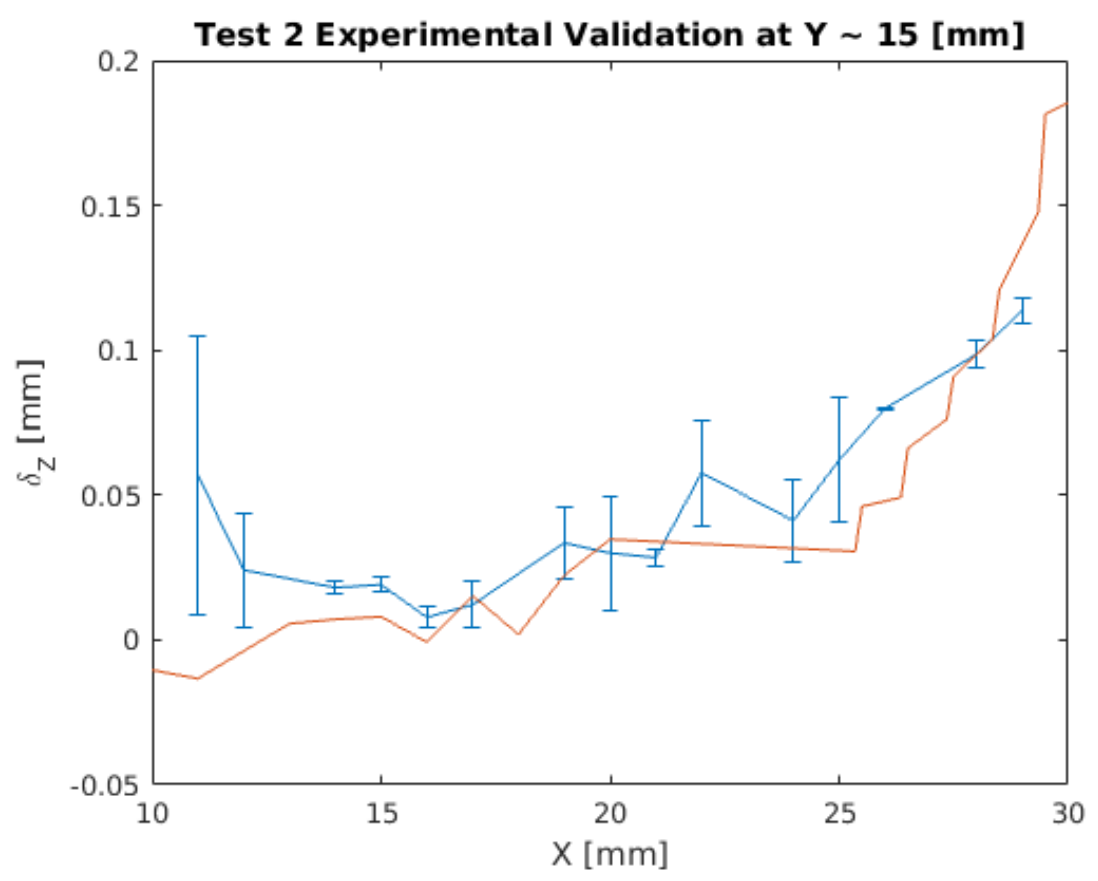

Figure A.8 - Test 2 Experimental Validation at $\mathrm{Y} \approx 15[\mathrm{~mm}]$

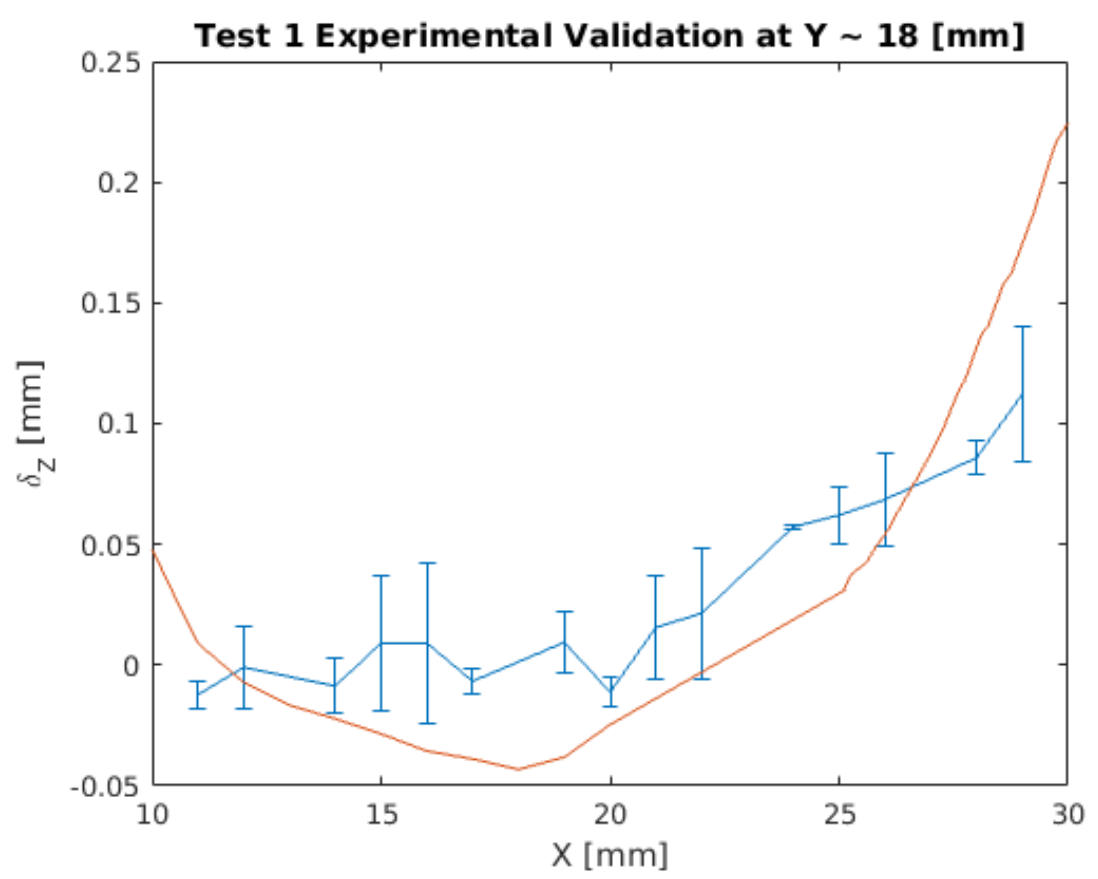

Figure A.9 - Test 1 Experimental Validation at $\mathrm{Y} \approx 18[\mathrm{~mm}]$ 
Test 2 Experimental Validation at $Y \sim 18[\mathrm{~mm}]$

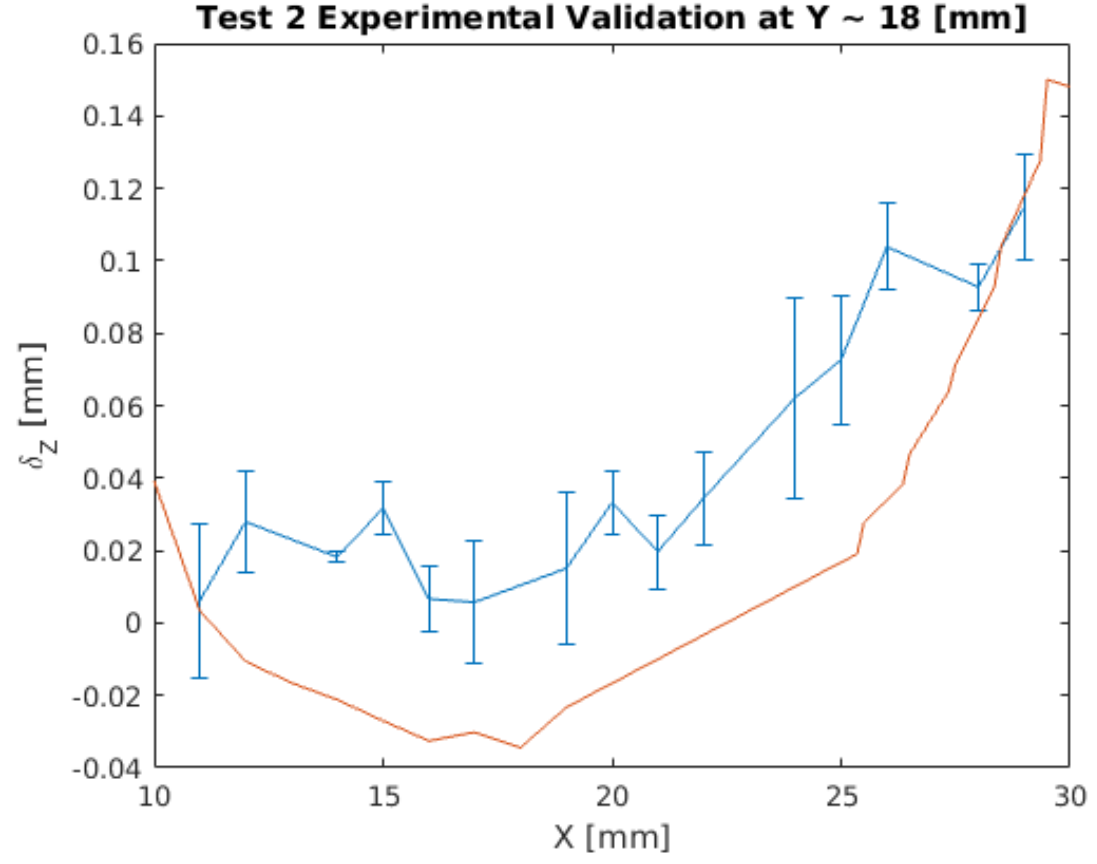

Figure A.10 - Test 2 Experimental Validation at $\mathrm{Y} \approx 18[\mathrm{~mm}]$ 